\title{
Mapping collective behavior in the big-data era
}

\author{
R. Alexander Bentley \\ Department of Archaeology and Anthropology, University of Bristol, Bristol \\ BS8 1UU, United Kingdom \\ r.a.bentley@bristol.ac.uk \\ http://www.alex-bentley.com \\ Michael J. O’Brien \\ Department of Anthropology, University of Missouri, Columbia, MO 65211 \\ obrienm@missouri.edu \\ http://cladistics.coas.missouri.edu \\ William A. Brock \\ Department of Economics, University of Missouri, Columbia, MO 65211; and \\ Department of Economics, University of Wisconsin, Madison, WI 53706 \\ wbrock@scc.wisc.edu \\ http://www.ssc.wisc.edu/ wbrock/
}

\begin{abstract}
The behavioral sciences have flourished by studying how traditional and/or rational behavior has been governed throughout most of human history by relatively well-informed individual and social learning. In the online age, however, social phenomena can occur with unprecedented scale and unpredictability, and individuals have access to social connections never before possible. Similarly, behavioral scientists now have access to "big data" sets - those from Twitter and Facebook, for example-that did not exist a few years ago. Studies of human dynamics based on these data sets are novel and exciting but, if not placed in context, can foster the misconception that mass-scale online behavior is all we need to understand, for example, how humans make decisions. To overcome that misconception, we draw on the field of discrete-choice theory to create a multiscale comparative "map" that, like a principal-components representation, captures the essence of decision making along two axes: (1) an east-west dimension that represents the degree to which an agent makes a decision independently versus one that is socially influenced, and (2) a north-south dimension that represents the degree to which there is transparency in the payoffs and risks associated with the decisions agents make. We divide the map into quadrants, each of which features a signature behavioral pattern. When taken together, the map and its signatures provide an easily understood empirical framework for evaluating how modern collective behavior may be changing in the digital age, including whether behavior is becoming more individualistic, as people seek out exactly what they want, or more social, as people become more inextricably linked, even "herdlike," in their decision making. We believe the map will lead to many new testable hypotheses concerning human behavior as well as to similar applications throughout the social sciences.
\end{abstract}

Keywords: agents; copying; decision making; discrete-choice theory; innovation; networks; technological change

\section{Introduction}

The 1960s' term “future shock” (Toffler 1970) seems ever more relevant today in a popular culture that seems to change faster and faster, where global connectivity seems to spread changes daily through copying the behavior of others as well as through random events. Humans evolved in a world of few but important choices, whereas many of us now live in a consumer world of almost countless, interchangeable ones. Digital media now record many of these choices. Doubling every two years, the digital universe has grown to two trillion gigabytes, and the "digital shadow" of every Internet user (the information created about the person) is already much larger than the amount of information that each individual creates (Gantz \& Reinsel 2011).

These digital shadows are the subjects of "big data" research, which optimists see as an outstandingly large sample of real behavior that is revolutionizing social science (Aral \& Walker 2012; Golder \& Macy 2011; Onnela \& Reed-Tsochas 2010; Ormerod 2012; Wu \& Huberman 2007). With all its potential in both the academic and commercial world, the effect of big data on the behavioral sciences is already apparent in the ubiquity of online surveys and psychology experiments that outsource projects to a distributed network of people (e.g., Rand 2012; Sela \& Berger 2012; Twenge et al. 2012). With a public already overloaded by surveys (Hill \& Alexander 2006; Sumecki et al. 2011), and an ever-increasing gap between individual experience and collective decision making (Baron 2007; Plous 1993), the larger promise of big-data research appears to be as a form of mass ethnography - a record of what people actually say and decide in their daily lives. As the Internet becomes accessible by mobile phone in the developing world, big data also offer a powerful means of answering the call to study behavior in non-Western societies (e.g., Henrich et al. 2010). 
But there is a downside to big-data research. Without clear objectives and a unifying framework, behavioral scientists may ask whether it is useful, for example, to infer from millions of Facebook pages or Twitter feeds that "men are more influential than women ... [and] that influential people with influential friends help spread" information (Aral \& Walker 2012) or that "people awaken later on weekends" (Golder \& Macy 2011). Big-data research runs the risk of merely reinforcing the most convenient "as if" assumptions about human behavior that currently divide the behavioral sciences (e.g., Gintis 2007; 2009; Laland \& Brown 2011; Mesoudi 2011; Mesoudi et al. 2006; Rendell et al. 2011). Such assumptions are often chosen to fit the purpose, either (a) at the economic end of the socialscience spectrum, where individual decision rules are optimized for the environment and maximize reproductive success or some utility function (Gintis 2007), or (b) at the cultural-historical end, where choices are programmed by broader social influences, "culture" (Davis \& Fu 2004), "norms" (Postmes et al. 2001), or "habitus" (Bordieu 1990).

R. Alexander Bentley is Professor and Head of the Department of Archaeology and Anthropology, University of Bristol. He is the author, along with Michael J. O'Brien and Mark Earls, of I'll Have What She's Having: Mapping Social Behavior (MIT Press, 2011). Much of his recent research, published in PLoS ONE, European Business Review, Frontiers in Psychology, Mind and Society, and Current Zoology, addresses the spread of information, especially in the online age. He also uses isotopic analysis of prehistoric skeletons to study social organization in Neolithic Europe and Southeast Asia. Recent publications appear in Antiquity and Proceedings of the National Academy of Sciences, USA.

Michael J. O'Brien is Professor of Anthropology and Dean of the College of Arts and Science, University of Missouri. His recent research takes three directions: (1) the dynamics of information flow in modern societies, in collaboration with R. Alexander Bentley and William A. Brock; (2) the role of agriculture in human niche construction, in collaboration with Kevin N. Laland (University of St Andrews); and (3) the first thousand or so years of human occupation of North America, in collaboration primarily with Mark Collard and Briggs Buchanan (Simon Fraser University). Recent publications appear in Current Anthropology, Journal of Archaeological Method and Theory, PLoS ONE, Journal of Archaeological Science, and Frontiers in Psychology.

William A. Brock is Vilas Research Professor Emeritus, University of Wisconsin, Madison, and Research Professor, University of Missouri. He is a Fellow of the Econometric Society (since 1974), and was a Sherman Fairchild Distinguished Scholar, California Institute of Technology, in 1978, and a Guggenheim Fellow in 1987. He has been a Fellow of the American Academy of Arts and Sciences since 1992, a member of the National Academy of Sciences, USA, since 1998, and a Distinguished Fellow, American Economic Association, in 2004. Brock received the honorary degree of Doctor Honoris Causa from the University of Amsterdam in January 2009.
The degree of social influence on decision making is an empirical question that underlies what big data mean and how they can be used. As an example of the importance of this issue, consider the ubiquitous reliance on crowdsourcing in behavioral studies, business, and politics (Horton et al. 2011) - what Wegner (1995) termed "transactive memory" and now commonly called the "wisdom of crowds" (Couzin et al. 2011; Lorenz et al. 2011; Surowiecki 2004): Ask a question of a group of diverse, independent people, and the errors in their answers statistically cancel, yielding useful information. Wikipedia is founded on this assumption, of course, even though copying of text is essential to its growth (Masucci et al. 2011).

The wisdom-of-crowds effect is lost, however, if agents are not thinking independently (Bentley \& O’Brien 2011; Salganik et al. 2006). There are numerous indications that online behavior may be getting more herdlike, more confused, or even more "stupid" (Carr 2008; Onnela \& Reed-Tsochas 2010; Sparrow et al. 2011). In economies replete with online communication and a constant barrage of information - often to the point of overload (Hemp 2009) - crucial human decision making might be becoming more herdlike in contexts such as voting (Arawatari 2009) and forming opinions about climate change (Ingram \& Stern 2007), mating (Lenton et al. 2008; 2009), music (Salganik et al. 2006), and finances (Allen \& Wilson 2003). Herdlike behavior could be worrisome, say, for those in the public-health and medical sectors (e.g., Bates et al. 2006; Benotsch et al. 2004; Zun et al. 2004).

How does one take advantage of big data, with its huge sample sizes and natural contexts, and still address the degree and nature of social influence among the contexts being studied? We introduce here a simple map of the different types and domains of human behavioral innovation-translated as "decision making"-that can be characterized directly from population-scale data. We view the map as analogous to a coarse-grained tool much like a Google map. We illustrate the "zoom" feature of the tool by using one major theory of human decision making: discrete-choice analysis (see McFadden [2001] and his references to creators of the theory [e.g., Kahneman, Tversky, and Luce]). We chose discrete-choice theory as an expository vehicle because it is related to many other theories of human decision making, both individually and in groups, such as replicator dynamics (Krakauer 2011), Bayesian updating and information theory (Krakauer 2011), and statistical mechanics (Durlauf 1999), as well as to empirical problems associated with measuring "social capital" (Durlauf 2002). We argue that our conceptual tool - a "reduced form" parameterization of the large research area of discrete-choice approaches to decision making - is useful in helping social scientists navigate these large areas of science just as the Google map tool is useful in navigating geographical areas at various levels of resolution.

\section{The map}

In the simplest of terms, the map (Figure 1) graphs two analytical dimensions: (1) the extent to which a decision is made independently or socially, and (2) the transparency or opaqueness of the decision in terms of payoff. The western edge of the map represents completely 


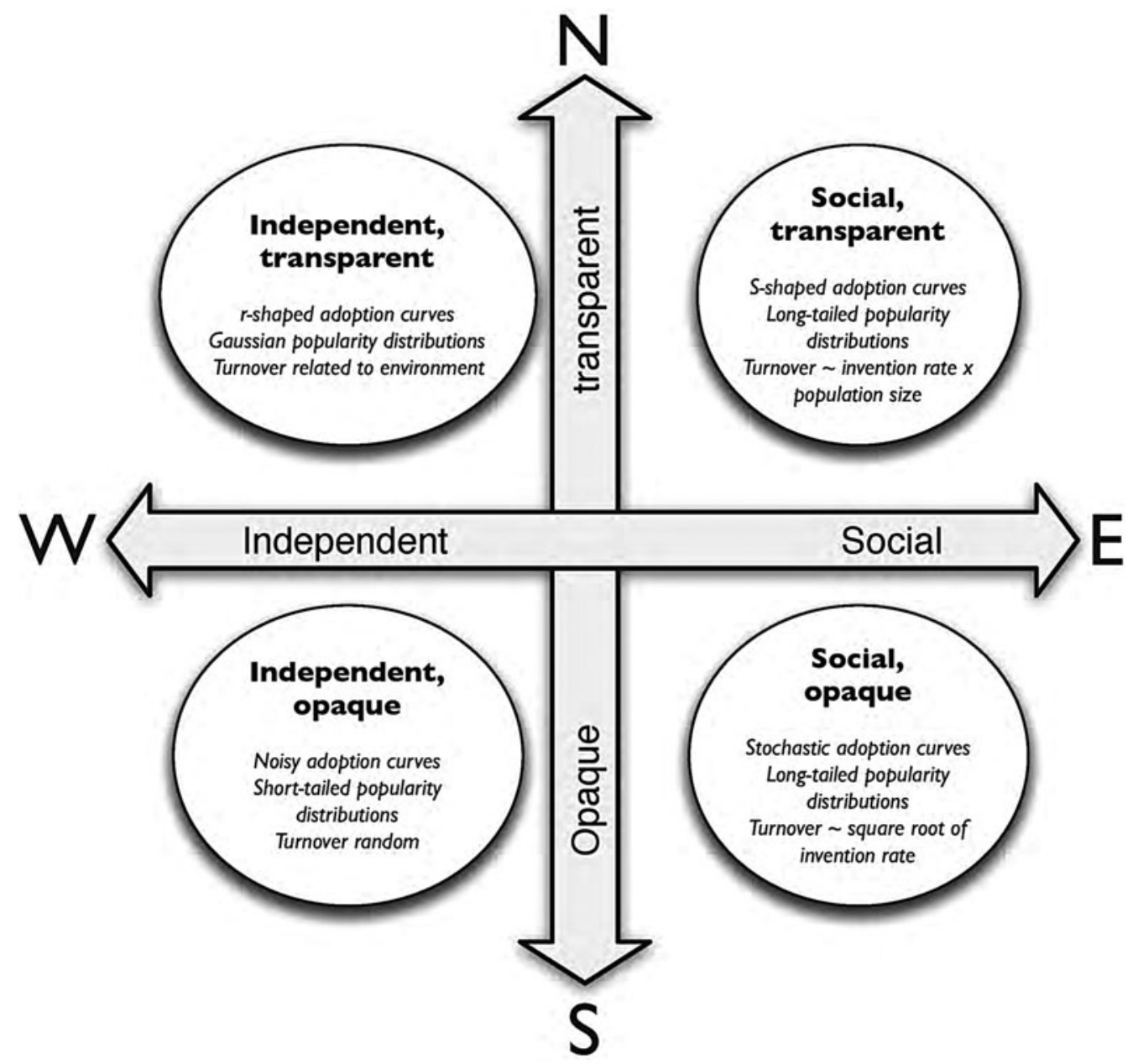

Figure 1. Summary of the four-quadrant map for understanding different domains of human decision making, based on whether a decision is made independently or socially and the transparency of options and payoffs. The characteristics in the bubbles are intended to convey likely possibilities, not certitudes.

independent decision making, where agents use no information from others in making decisions, and the eastern edge represents pure social decision making, where agents' decisions are based on copying, verbal instruction, imitation, or other similar social process (Caldwell \& Whiten 2002; Heyes 1994). The north-south dimension of the map represents a continuum from omniscience to ignorance, or - more formally - the extent to which there is a transparent correspondence between an individual's decision and the consequences (costs and payoffs) of that decision. The farther north we go on the map, the more attuned agents' decisions will be with the landscape of costs and payoffs. As we move south, agents are less and less able to discern differences in potential payoffs among the choices available to them.

The map is considerably more than a qualitative description, as it is grounded in established discrete-choice approaches to decision making. If we start with the full version, which we simplify below, we have the equation

$$
\begin{aligned}
& P_{t}(k)=\frac{1}{Z_{t}} \mathrm{e}^{b_{t} U\left(x_{k t}, J_{t} \bar{P}_{t}(k)\right)} \\
& Z_{t} \equiv \sum_{j=1}^{N_{t}} \mathrm{e}^{b_{t} U\left(x_{j t} J_{t} \bar{P}_{t}(j)\right)}
\end{aligned}
$$

where there are $N_{t}$ possible choices at date $t$, and $\bar{P}_{t}(k), b_{t}, U\left(x_{k t}, J_{t} \bar{P}_{t}(k)\right)$ denote: (1) the probability that choice $k$ is made at date $t$; (2) the "intensity of choice," which is inversely related to a standard-deviation measure of decision noise in choice and is positively related to a measure of transparency (clarity) of choice; and (3) the deterministic payoff of choice $k$ at date $t$. The deterministic payoff is a function, $U\left(x_{k t}, J_{t} \bar{P}_{t}(k)\right)$, of a list of covariates, $x_{k t}$, that influence choice at date $t . \bar{P}_{t}(k)$ denotes the fraction of people in a relevant peer or reference group that choose option $k$, and $J_{t}$ denotes a strength of social-influence parameter that the fraction of people, $\bar{P}_{t}(k)$, in an individual's peer group (sometimes called "reference group") has on the person (agent) under study. Subscripts appear on variables because their values may change over time, depending on the dynamical history of the system.

The map allows us to operate with only two parameters extracted from Equation (1) above: $J_{t}$ and $b_{t}$. The eastwest dimension of the map represents $J_{t}$ - the extent to which a decision is made independently or socially. The western edge, representing completely independent learning, corresponds to $J_{t}=0$ in mathematical notation. Conversely, the eastern edge, pure social decision making, corresponds to $J_{t}=\infty$. In between the extremes is a sliding scale in the balance between the two. This is a flexible 
measure in terms of the agents represented. The midpoint could represent, for example, a population of half social learners and half individual learners, or each individual giving a 50\% weight to his or her own experience and a likewise amount to that of others. Location along the east-west dimension may not always affect the equilibrium toward which each behavior evolves, but it will certainly affect the dynamics by which that equilibrium is approached.

The north-south dimension of the map represents $b_{t}-$ the extent to which there is a transparent correspondence between an individual's decision and the consequences of that decision. The farther north we go on the map, the more attuned agents' decisions will be with the landscape, which we can represent by the function $U(. .$.$) of costs and$ payoffs. At the extreme northern end are behaviors where there is an immediately detectable impact of getting a decision right or wrong. It corresponds to $b_{t}=\infty$. As we move south, behavioral evolution can begin to create an unconstrained set of possible solutions, meaning there are fewer and fewer reasons for one solution to be preferred over another. The farthest south one can go corresponds to total indifference, which is where $b_{t}=0$, and the probability of any particular choice among $N_{t}$ possible choices approaches zero (because $1 / N_{t}$ goes to zero as $N_{t}$ goes to infinity). Choices in the southern extreme of the map need not be trivial, as this end also represents cases where people are poorly informed about their choices and perhaps overwhelmed by decision fatigue-for example, when the number of choices, $N_{t}$, is very large, or when agents are otherwise unable to discern differences in potential payoffs among the choices available to them (Baumeister \& Tierney 2011; Sela \& Berger 2012). As the number of options grows, a natural way to try to minimize the cognitive cost of choosing among them would be to simply copy the choices of more-experienced choosers.

In terms of $J_{t}$ and $b_{t}$, we now have a four-quadrant map on which the extreme northwest is $\left(J_{t}, b_{t}\right)=(0, \infty)$, the extreme southwest is $\left(J_{t}, b_{t}\right)=(0,0)$, the extreme northeast is $\left(J_{t}, b_{t}\right)=(\infty, \infty)$, and the extreme southeast is $\left(J_{t}, b_{t}\right)=$ $(\infty, 0)$. In addition to characterizing the quadrants in terms of $J_{t}$ and $b_{t}$, we can characterize them in terms of empirical signatures amenable to big-data analysis. ${ }^{1}$ Our estimations are displayed in Figure 2. The default assumption for many social scientists is the normal distribution - shown in the northwest quadrant in Figure 2a-but there are others, including the negative-binomial distribution, which typifies the southwest quadrant, as well as highly rightskewed, "long-tailed" distribution, which is consistent with phenomena on the eastern half of the map (Fig. 2a). ${ }^{2}$ Figure $2 \mathrm{~b}$ shows the same distributions plotted as cumulative functions, which accommodate different forms that data might take (cumulative distributions are especially useful for smaller data-sets so that histogram binning is not an issue).

In general, the farther south on the map we go, the noisier and less predictable the time series are for the different options. In the northwest, the time series are essentially flat, except when a new discovery is made and adopted according to a rapidly rising $r$-curve (Fig. 2a, northwest). In the $\left(b_{t}, J_{t}\right)$ discrete-choice setting, this would correspond to adding a new option, call it $N_{t}+1$, to the original $N_{t}$ options, where the payoff of the new option is larger than that of any of the original options. Given that $b_{t}$ is large in the northwest, we would expect rapid movement toward the new and superior option. (a)

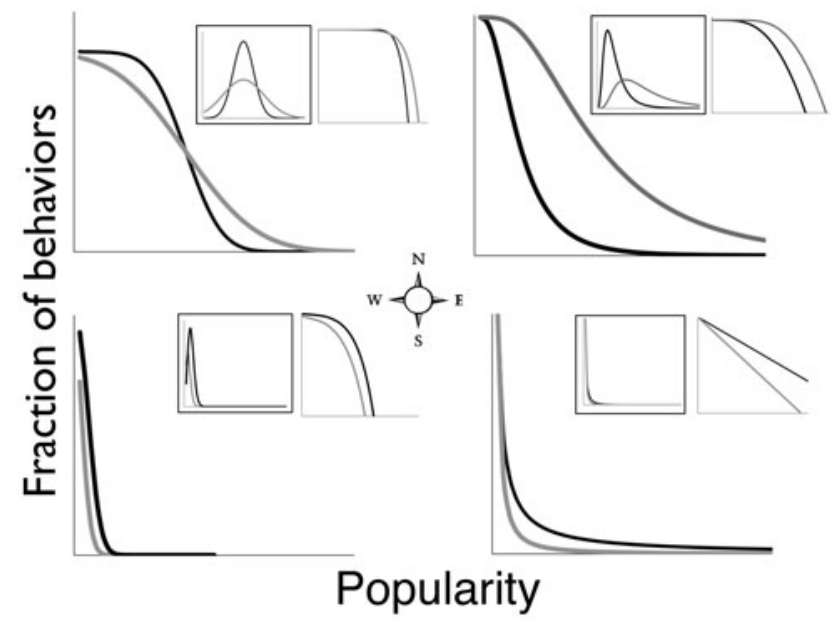

(b)

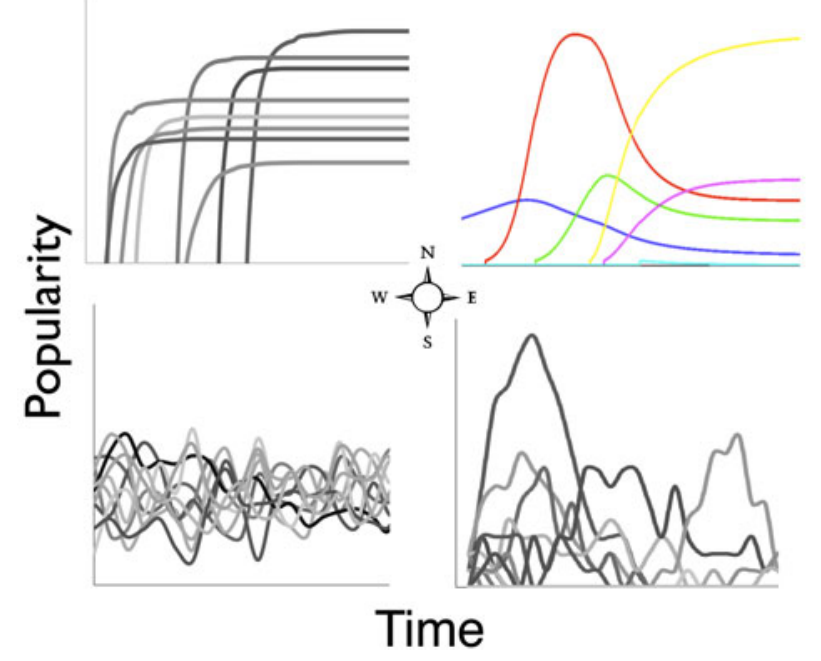

Figure 2. (a) Generalized distributions characterizing the different map quadrants: normal (Gaussian) in the northwest, negative binomial in the southwest, log-normal in the northeast, and power law in the southeast. Each plot shows popularity of a choice on the $x$-axis versus cumulative probability of having at least that popularity on the $y$-axis. The left, boxed insets show the non-cumulative fraction of choices on the $y$-axis that have the popularity indicated on the $x$-axis; the right, unboxed insets show the same cumulative distributions but with doublelogarithmic axes. (b) Representative timelines for the popularity of different options, for each quadrant. Note that the different lines plotted for the northwest quadrant represent different payoffs, such that curves with a lower $y$-intercept at asymptote represent lower payoff/cost decisions than those with higher intercepts. For the northeast quadrant, the curves (after Kandler \& Laland 2009) represent innovations adopted at different rates and subsequently declining in popularity to levels commensurate with their real-world utility.

Indeed, as $b_{t}$ becomes very large (approaching infinity), all choice jumps as fast as possible to the new option. In the northeast, these behaviors are adopted through social diffusion, which takes the shape of an S-curve, but over the long term, the result is similarly flat timelines (Fig. 2a, northeast). In the northeast, the system can get stuck on inferior alternatives if the social influence to conform to a previously popular choice is strong enough relative to the gain to switching even when the intensity 
of choice is large (Brock \& Durlauf 2001b). In contrast to the north, timelines in the south show turnover in the most popular behavior, either dominated by random noise (Fig. 2a, southwest) or stochastic processes (Fig. 2a, southeast). Turnover in the composition of long-tailed distributions is a fairly new discussion (e.g., Batty 2006; Evans \& Giometto 2011), as much of the work in the past century has considered the static form of these distributions.

The map requires a few simplifying assumptions to prevent it from morphing into something so large that it loses its usefulness for generating potentially fruitful research hypotheses. First, it treats the various competencies of agents (intelligence, however measured; education, motor, and cognitive skills; and so on) as real but too finegrained to be visible at the scale of data aggregated across a population and/or time. Second, agents are not assumed to know what is best for them in terms of long-term satisfaction, fitness, or survival (even rational agents, who are very good at sampling the environment, are not omniscient). Third, we blur the distinction between learning and decision making. Technically, they are separate actions, but this distinction draws too fine a line around our interest in what ultimately influences an agent's decision and how clearly the agent can distinguish among potential payoffs. Fourth, although the map represents a continuous space of $b_{t}$ and $J_{t}$, we divide it into quadrants for ease of discussion and application to example datasets. Importantly, our characterizations are based on extreme positions of agents within each quadrant. As agents move away from extremes, the characterizations are relaxed.

\subsection{Northwest: Independent decision making with transparent payoffs}

The northwest quadrant contains agents who make decisions independently and who know the impact their decisions will have on them. The extreme northwest corner is where rational-actor approaches and economic assumptions (Becker 1976; 1991) - for example, that individuals will always choose the option that provides the best benefit/cost ratio - most obviously and directly apply. Although we cite Becker (1976), especially his Treatise on The Family (Becker 1991), as examples of research work on the northwest, we also note Becker's (1962) article in which he shows that many predictions of economic rational-actor theory that would appear in the northwest quadrant (e.g., downward-sloping demand curves) still hold when agents are irrational and simply choose their purchases at random, subject to budget constraints - a behavior found in the southwest quadrant. This is the continuous-choice analog of $b_{t}=0$ in a discrete-choice model.

We put Kahneman-type bounded-rationality theories (e.g., Kahneman 2003) in the northwest because they emphasize actual cognitive costs of information processing and other forces that are rational responses to economizing on information-processing costs and other types of costs in dealing with decision making in a complex world. One of many examples of empirical patterns in the northwest is the "ideal-free distribution," which predicts the pattern of how exclusive resources are allocated over time through individual agents seeking the best resource patches (e.g., Winterhalder et al. 2010). Reward-driven trial and error and bounded rationality contribute to powerful hillclimbing algorithms that form the mechanism delivering the fitness-maximizing behaviors predicted by models of microeconomics and human behavioral ecology (e.g., Dennett 1995; Mesoudi 2008; Nettle 2009; Winterhalder \& Smith 2000).

It is precisely these algorithms that also begin to move individuals out of the extreme northwest corner and into other areas of the map. This is why we state repeatedly that although we categorize each quadrant with a certain kind of behavior, they represent extremes. An example of a type of dynamic-choice mechanism that fits in the northwest quadrant, but toward the center of the map, is replicator dynamics,

$$
\frac{\mathrm{d} P_{k}}{\mathrm{~d} t}=b P_{k}\left(U_{k}-\sum_{j=1}^{N} P_{j} U_{j}\right), \quad k=1,2, \ldots N,
$$

where $U_{k}$ is the payoff to choice $k$ (called "fitness" of choice $k$ in the evolutionary-dynamics literature), $P_{k}$ is the fraction of agents making choice $k$, and $b$ measures the speed of the system in reaching the highest fitness peak, that is, the best choice (Krakauer 2011; see also Mesoudi 2011). Here, $b_{t}$ plays a role similar to what it does in the discrete-choice model: It measures the "intensity" of adjustment of the replicator dynamics toward the highest fitness choice. It is easy to introduce social effects into the replicator dynamics by adding the term $J P_{k}$ to each $U_{k}$.

2.1.1. Patterns in the northwest. In the northwest quadrant, the popularity of variables tends to be normally (Gaussian) distributed as a result of cost/benefit constraints underlying them. ${ }^{3}$ In terms of resource access, the northwest is exemplified by the "ideal-free distribution," which predicts the static, short-tailed distribution of resource access per agent through time, as individual agents seek the best resource patches (e.g., Winterhalder et al. 2010). In terms of behavior, the northwest implies that the maximal behavior should become the most popular option and remain so until circumstances change or a better solution becomes available. As the new behavior is selected, choices in the northwest will thus have either a stable popularity over time (stabilizing selection) or a rapidly rising $r$-curve (Fig. $2 \mathrm{~b}$, northwest). The sizes of human tools and equipment - handaxes, pots of a certain function, televisions-are normally distributed and located in the northwest quadrant because humans select tools to fit the constraints of the purpose (Basalla 1989). The same is true of the market price of a product or service (Nagle \& Holden 2002), daily caloric intake (Nestle \& Nesheim 2012), culturally specific offers in the Ultimatum Game (Henrich et al. 2005), numerical calculations (Hyde \& Linn 2009; Tsetsos et al. 2012), and ratings of attractiveness by body-mass index (George et al. 2008). If these constraints change over time, the mean of the normal distribution shifts accordingly.

\subsection{Northeast: Socially based decision making with transparent payoffs}

As opposed to the northwest, where individuals recognize new beneficial behaviors and make decisions on their own, behaviors spread socially in the northeast quadrant. Once they learn about a new behavior, through any number of social processes (Laland 2004; Mesoudi 2011), agents along the northern edge clearly understand the 
rationale for adopting it in terms of payoff. As the transparency of payoffs begins to blur, however, there is less and less conscious weighing of options and more use of heuristics-efficient cognitive processes, whether conscious or unconscious, that focus only on a portion of the available information. One heuristic is to simply copy what others are doing, whether it is copying the majority (Laland 2004) or copying the behaviors of individuals with the most skill or prestige (Atkisson et al. 2012; Henrich \& Gil-White 2001).

When decisions are based on either success or perceived fitness, eventual outcomes will parallel those of the northwest; human behavioral ecologists call this the "phenotypic gambit" (e.g., Low 2001; Nettle 2010). The northeast quadrant can therefore apply on a longer time scale, in which an adaptive equilibrium is reached by social-learning processes. Different culture-specific mean offers in the Ultimatum Game, for example, reflect the costs and benefits of group adaptation in a wide range of different environments (Henrich et al. 2006). As long as there is some individual learning and decision making going on within a population-that is, anywhere but along the extreme eastern edge of the map - the eventual outcome can be the same as if all learning and decision making were independent. Along the extreme eastern edge, where there is no independent learning at all to inform the socially learned (imitated) practices in circulation, adaptive potential to an exterior environment is lost. For example, fishermen who always copy other, perhaps more successful fishermen can get stuck in a poorer part of a fishery and fail to locate better areas (Allen \& McGlade 1986). Efficient communal fishing requires some individual boats to randomly probe other areas of a fishery than the ones that look apparently the best based on past catch experience (see Mesoudi 2008).

2.2.1. Patterns in the northeast. Along the northern edge of the northeast quadrant, population size affects the efficiency with which agents learn and retain new and better behavioral strategies (Henrich 2010; Shennan 2000). This northeast pattern results from plotting the sizes of sample populations, $N_{\text {pop, I }}$, on the $x$-axis and the number of tools or inventions in those populations on the $y$-axis. A linear correlation between these variables is predicted for small-scale, adaptive societies (Henrich 2010), which was demonstrated empirically by the number of tools recorded on different Oceanic islands at early European contact (Kline \& Boyd 2010). This pattern distinguishes the northeast, as in the other three quadrants population size should not affect the likelihood of adaptive innovations (e.g., Bentley \& O'Brien 2011; Bentley et al. 2007; Henrich 2010).

One pattern that is consistent with behavior along the far eastern edge of the map is the breakdown of the Law of Large Numbers and the Central Limit Theorem. The idea is this: As the product of the intensity of choice and the strength of social interactions, $b_{t} J_{t}$, grows larger than some threshold, one can show (Amaro de Matos \& Perez 1991; Brock \& Durlauf 2001b; Repetto 2006) that the Central Limit Theorem underlying the Gaussian distribution breaks down, and more-complicated distributions mixtures of Gaussian distributions - appear. This behavior is consistent with the east side of the map because it can't happen unless there is positive social influence. However, it can happen when social influence is weakly positive but intensity of choice is high enough so that the product of social influence and intensity of choice exceeds the critical threshold that causes the breakdown of the Central Limit Theorem. Intuitively, what is happening here is a pile-up of correlated behaviors caused by the interaction of social influences coupled with strong enough intensity of choice, which can become large enough to prevent the familiar "washing out" of weakly correlated or zero-correlated effects.

A simple model for this resembles the Gaussian model except that agents do not judge directly which behavior is best but rather which behavior is most popular. If agents copy with a probability proportional to the existing popularity, but with some error, the result should be a log-normal distribution of popularity levels (Fig. 2a, northeast). This is a common pattern, and established models ${ }^{4}$ of proportionate advantage (or "preferential attachment" for networks) assume that the popularity of a choice in the current time slice is proportional to its popularity at the previous time slice multiplied by some growth rate normally distributed over time (e.g., Adamic \& Huberman 2000; Huberman \& Adamic 1999; Stringer et al. 2010; Wu \& Huberman 2007). The result is a log-normal distribution of the accumulated popularity that spreads outward through time on a logarithmic scale, such that turnover is fairly minimal-the most popular choices tend to remain popular (Fig. 2, northeast).

\subsection{Southeast: Social decision making without transparent payoffs}

The southeast quadrant combines the lack of transparency of payoffs found in the south with the social learning of the east. This is the part of the map where, for the discretechoice area, $J_{t}$ is large and $b_{t}$ is small. It stimulates the researcher to try to uncover and measure processes that cause the choice system to be located in this quadrant, that is, where social forces are strong and the intensity of choice across available options is small. The low intensity of choice may be the result of a large standard deviation of the random elements in the choice process, and that, in turn, may be a result of a lack of information about the choices relative to the differences in underlying values of the choices. The farther south we go, the less transparent payoffs become. Just to the south of the "equator," agents might lack knowledge of the benefits of the behavior itself, or even of the qualifications of the people they might learn from, so they imitate based solely on popularity (frequency-dependent decisions) (Eriksson et al. 2007). In the extreme southeast, not only are the options themselves equivalent (as in the southwest), but so too are the people who potentially serve as models. It is as if each person points to someone else and says, "I'll have what she's having" (Bentley et al. 2011).

The null model for the southeast is imitating others as if it were done randomly, which is also known as the neutral model because there is an ignorance - a neutrality - in saying, "I'll have what she's having." As we discuss below, the neutral model usually requires some minority of independent learning to fit the data, so rarely do real-world situations plot along the extreme eastern edge of this quadrant, which is reserved for complete herding, where everyone imitates someone else (e.g., Helbing et al. 2000). 
Another type of behavior that one might argue belongs in the southeast is confirmation bias plus weak feedback loops (Strauss 2012). Strong feedback loops induce rapid learning toward the best choice, but weak feedback loops do the opposite. Confirmation bias is a form of mistaken choice and/or mistaken belief that requires repeated challenge and strong immediate feedbacks to change, even though it may be wrong, and perhaps very wrong. Although it is true that confirmation bias and weak feedback loops have nothing to do with social pressures per se, Strauss's (2012) "filter bubble" - one of six reasons he sees for increasing polarization among U.S. voters - is a channel through which the Internet can reinforce one's own confirmation bias from "linking" that individual to other Internet users with similar preferences. This force could act "as if" it were an increase in peer-group social-influence strength, $J_{t}$.

2.3.1. Patterns in the southeast. In the extreme southeast, socially based decisions are made, but payoffs among different options completely lack transparency. In this extreme, a simple null model is one in which agents copy each other in an unbiased manner-not intentionally copying skill or even popularity. For maximum parsimony, this can be modeled as a process of random copying. This is not to say that agents behave randomly; rather, it says that in the pattern at the population scale, their biases and individual rationales balance out, just as the errors balance out in the other quadrants. It is "as if" agents are ignorant of the popularity of a behavior.

One version of the unbiased-copying model (e.g., Mesoudi \& Lycett 2009; Simon 1955; Yule 1924) assumes that $N_{\text {pop }}$ agents make decisions in each time step, most of whom do so by copying another agent at random - not another option at random, a behavior that belongs in the southwest, but copying another agent's decision. This model also uses the individual learning variable, $\mu$. Varying this parameter shifts the longitude on the map $(\mu$ can be seen as a distance from the eastern edge at $\mu=0$, with $\mu=100 \%$ at the extreme western edge). In the core of the southeast, $\mu$ is usually rather small, say, $5 \%$ of agents choosing a unique, new variant through individual learning. This model can be translated, in a mathematically equivalent way, from populations to individuals by effectively allowing previous social-learning encounters to populate the mind, so to speak. In this Bayesian learning model, social-learning experiences are referenced in proportion to their past frequency (such as number of times a word has been heard), with occasional unique invention (Reali \& Griffiths 2010).

Unbiased-copying models predict that if we track individual variants through time, their frequencies will change in a manner that is stochastic rather than smooth and continual (northwest and northeast) or completely random (southwest). The variance in relative popularity of choices over time should depend only on their prior popularity and on the population size, $N_{\text {pop }}$. If we use evolutionary drift as a guide, the only source of change in variant frequencies, $v$, over time is random sampling, such that the variance in frequencies over time is proportional to $v(1-v) / N_{\text {pop }}$ (Gillespie 2004). The popularity is thus stochastic, with the only factors affecting popularity in the next time step being the current popularity and population size. Unbiased-copying models yield highly right-skewed, or long-tailed, distributions of popularity, as shown in Figure 2a (southeast).

This means that turnover (Fig. 2b, southeast) is a diagnostic difference from the northeast quadrant, in that in the southeast the accumulation of innovations - the cultural "ratchet" (Tomasello et al. 1993) - should not correlate strongly with population size in the southeast (Bentley et al. 2007; Evans \& Giometto 2011). As Bettinger et al. (1996) point out, although there are $\mu N_{\text {pop }}$ inventions per generation in large populations, "the rate at which they will become fixed is an inverse function of $N_{\text {pop. }}$. [T] he two exactly cancel, so that . . . the turnover rate is just the reciprocal of the innovation rate" (p. 147). Although the problem becomes more complicated if the variants are ranked by frequency, such as a "top 10 most-popular list," the result is essentially the same. As long as a list is small compared with the total number of options (top 10 out of thousands, for example), the list's turnover is continual and roughly proportional to the square root of $\mu$, that is, it is not strongly affected by population size (Eriksson et al. 2010; Evans \& Giometto 2011).

\subsection{Southwest: Individual decision making without transparent payoffs}

The southwest quadrant, where agents interact minimally and choose from among many similar options, is characterized by situations confronted individually but without transparent payoffs. In other words, it is as if agents were guessing on their own. Although this may be a rare situation for individuals in subsistence societies, it may well apply pervasively to modern Western society, where people are faced with literally thousands of extremely similar consumer products and information sources (Baumeister \& Tierney 2011; Bentley et al. 2011; Evans \& Foster 2011; Sela \& Berger 2012). One candidate phenomenon, however, is entertainment, as preferences can vary such that choices at the population level appear to be random (we present an example in the next section). If hurried decisions are biased toward the most recent information (Tsetsos et al. 2012), for example, the outcome may appear "as if random" with respect to payoffs. In consumer economics, an effective, practical assumption can be that "purchase incidence tends to be effectively independent of the incidence of previous purchases ... and so irregular that it can be regarded as if random" (Goodhardt et al. 1984, p. 626, emphasis in original).

2.4.1. Patterns in the southwest. Whereas the null model for the southeast is imitating others as if it were done randomly, the null model for the southwest is choosing options. In the southwest quadrant, we expect popularity to be governed by pure chance (Ehrenberg 1959; Farmer et al. 2005; Goodhardt et al. 1984; Newman 2005). In short, the probability of any particular choice becoming popular is essentially a lottery, and as this lottery is continually repeated, the turnover in popularity can be considerable (Fig. 2b, southwest). Ehrenberg (1959) provided an idealized model for the guesswork of the southwest quadrant, where the distribution of popularity follows the negative binomial function, ${ }^{5}$ in which the probability falls off exponentially (Fig. 2a, southwest). Choices made by guesswork are uncorrelated, yielding a diagnostic pattern, as the exponential tail of the distribution is a sign that events are independent of one another (e.g., Frank 2009). 


\section{Is human decision making drifting to the southeast?}

We expect that, in the big-data era, certain aspects of decision making have moved to the southeast, especially given the exponential increase in information and interconnected population sizes (Beinhocker 2006; Bettencourt et al. 2007; Hausmann et al. 2011). In the subsections below, we consider three examples: language and ideas, relationships, and wealth and prestige.

\subsection{Language and ideas}

Considering the deep evolutionary roots of human sociality, it is not surprising that social learning (northeast) is how small-scale societies have adapted and accumulated technical knowledge for most of human existence (Apicella et al. 2012; Henrich et al. 2001; 2005; Hill et al. 2011). Clearly, the ratchet of cumulative cultural evolution (Tomasello et al. 1993) requires a balance of individual and social learners and decision makers (Mesoudi 2008; Rendell et al. 2011). Often, a small amount of informed individual learning goes a long way, amplified by a majority context of social learning, even among animals (e.g., Couzin et al. 2005). This mix is essentially a description of the northeast quadrant, with $b_{t}$ set to high transparency and $J_{t}$ set at a level of mainly social learning.

As technology has evolved and become something that is cumulative, it has become a phenomenon of the northeast quadrant. In the northeast, if social learners copy the best strategies and thereby make the best decisions (Bentley \& O'Brien 2011; Henrich 2004; Mesoudi 2008), larger populations will find better technologies faster because there are more individual learners producing information, assuming that individual learners are a fixed proportion of the population.

As another practical measure, increased population size, $N$, should correlate positively with the rate of new innovations, which has been shown to be linear in small-scale societies (Kline \& Boyd 2010). In modern cities, however, the correlation has become superlinear, where the number of inventions grows proportional to $N^{1.24}$ (Bettencourt et al. 2007b). Bettencourt et al. suggest two alternative explanations for this superlinearity: either inventors are individually more productive in a larger city or there are a disproportionate number of inventors in larger metropolitan areas. Both explanations seem consistent with location in the northeast. If it is the former, then most likely inventors are able to take advantage of more information and not be overwhelmed by it. If it is the latter, then it is still a northeastern pattern, only with a higher proportion of individual learners in the population.

Changing patterns may signal a shift toward the southeast with increased population densities and urbanism. In 1900, approximately $13 \%$ of the world's population lived in urban areas. That figure reached $29 \%$ in 1950 and $49 \%$ in 2005 , and it is expected to rise to $60 \%$ by 2030 (United Nations 2006). This increase in density could lead human populations away from optimality, in contrast to naturally selected relationships, such as the way the heart rate of organisms shows an optimized, inverse-scaling relationship with body size. As Bettencourt et al. (2007a) observed, there is no such optimization for a modern city, where walking speed scales with population size: The pace of urban life increases with city size in contrast to the pace of biological life, which decreases with organism size.
Of course, as stressed by Romer (2012) and many others, institutions must support innovation, otherwise a large population density does not matter. In other words, when population size is large, individuals on their own cannot search the full space of ideas and must rely on institutions (or, more recently, on search engines) to ensure transparency. As transparency decreases and social influence increases through a combination of inexpensive sociallearning opportunities and multiple similar options, there are now ubiquitous highly right-skewed popularity distributions with continual turnover of contemporary commercial markets (Beinhocker 2006).

Now, in the big-data era, the process of science and invention - the creation of ideas - has become well-documented. If science proceeds ideally (Kitcher 1993), it ought to plot in the northeast, where social learning is well-informed and seminal works by academic leaders are selectively adopted and developed by followers (Rogers 1962). Indeed, bibliometric studies show the most successful (highly cited) scientific mentors are those who train fewer protégés (Malmgren et al. 2010), which confirms high $J_{t}$ (more social interaction) together with high $b_{t}$ (more transparency). Also consistent with characteristics of the northeast, citations to scientific articles, and also patents, exhibit a highly right-skewed distribution with only slow turnover in the ranked popularity of citation rates (Bentley \& Maschner 2000; de Sola Price 1965; Stringer et al. 2010). This regularity also applies to the keywords used, as Figure 3 shows for a tradition of (a)

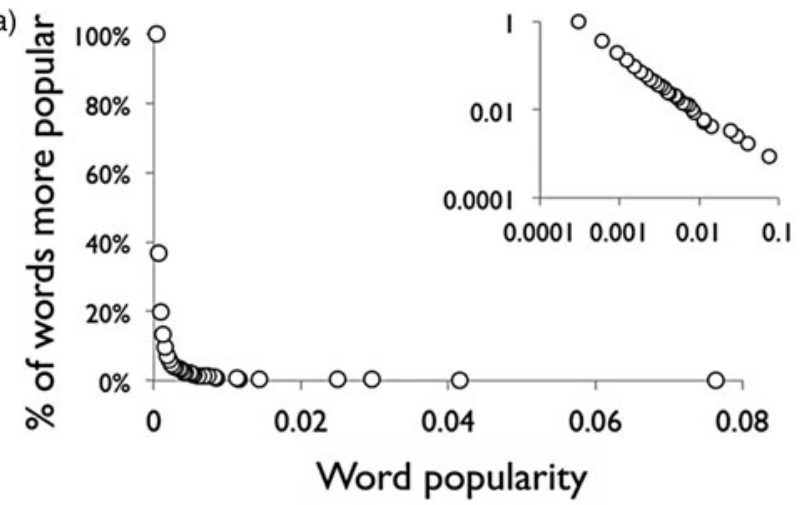

(b)

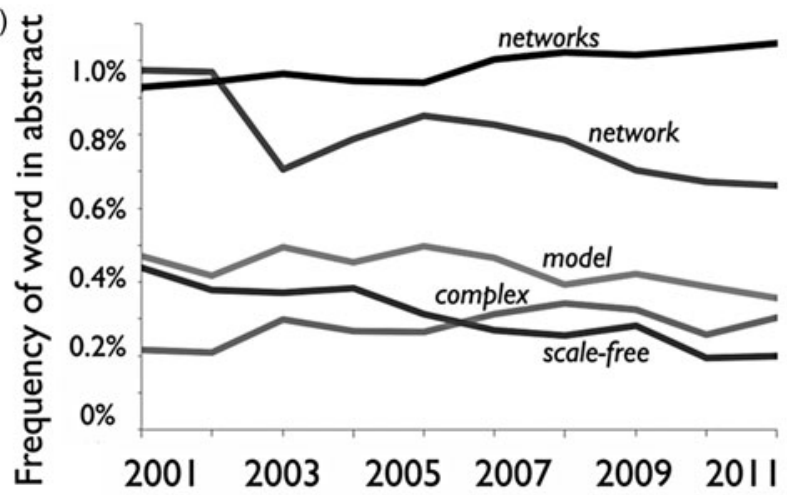

Figure 3. Distribution and turnover of keywords among all the articles citing a certain seminal article (Barabási \& Albert 1999): (a) Timelines of relative frequencies (number of keyword appearances divided by total number of words for the year), using the top five keywords of 2005 (logarithmic $y$-axes); (b) cumulative frequency distributions of all keywords. Open circles show distribution for 2001 and filled circles for 2005 . 
academic papers. Note that the distribution of word frequencies is log-normal but the turnover within the most popular keywords has been minimal.

Further support of scientific publishing's being a phenomenon of the northeast comes from Brock and Durlauf's (1999) use of the binary discrete-choice framework to examine potential social effects on the dynamics of "Kuhnian paradigm shifts" when evidence is accumulating against a current paradigm. Instead of the rapid acceptance of the new paradigm depicted in the northwest time-series plot of Figure 2b, Brock and Durlauf show that social pressures can easily lead to "sticky" dynamics of popularity around the old paradigm but then to a burst of speed toward the new paradigm once a threshold is passed. Consider a population, $N_{\text {pop }, t}$, of scientists, each of whom takes a position on an academic debate. We can represent this by a continuum that is divided into equal-sized "bins." If $J_{t}=0$, meaning that there are no social influences and each academician makes up his or her own mind independently, we might expect a normal distribution centered on the median (mean) academician. As transparency, $b_{t}$, increases (moves northward), the spread of the distribution narrows. As social influence, $J_{t}$, increases (moves eastward), the distribution can become bimodal or even multimodal - a mix of normal distributions centered at different means. The map, therefore, leads us to investigate the strength of social influences whenever we see evidence of polarization, that is, evidence of multimodal distributions.

Not surprisingly, compared to more-transparent scientific usage of words necessary to communicate new ideas, the public usage of language is more prone to boom-and-bust patterns of undirected copying in the southeast quadrant. Using raw data in Google's freely available files, we obtained the yearly popularity data for a set of climatescience keywords such as biodiversity, global, Holocene, and paleoclimate (established against a baseline of exponential growth in the number of words published over the last 300 years, a rate of about $3 \%$ per year). As we show (Bentley et al. 2012), most of the keywords fit the social-diffusion model almost perfectly. Indeed, almost all of them are becoming passé in public usage, with turnover suggestive of the southeast. Conversely, when we examined the narrow realm of climate-science literature, we found it plots farther north, as its keywords are not nearly subject to the same degree of boom and bust as in the popular media, with a consistency similar to that shown in Figure 3.

With the exceptional changes of online communication and texting, could language itself be drifting into the southeast? Perhaps it has been there for a long time; Reali and Griffiths (2010) suggest that the best null hypothesis for language change is a process analogous to genetic drift - "a consequence of being passed from one learner to another in the absence of selection or directed mutation" (p. 429). We see at least some southeast patterning in published language. By at least 1700 , the frequency of words published in English had come to follow a power law, now famously known as Zipf's Law (Clauset et al. 2009; Zipf 1949). There is regular turnover among common English words (Lieberman et al. 2007), but turnover among the top 1,000 most-published words seems to have leveled off or even slowed between the years 1700 and 2000, despite the exponential rise in the number of published books (Bentley et al. 2012). This deceleration of turnover with growing effective population is intriguing, as it is not expected for the northeast. A southeast trend is also indicated as online language is copied with much less transparency regarding the quality of the source (Bates et al. 2006; Biermann et al. 1999). This would, then, be a long way from the origins of language, which presumably began in the northwest, as straightforward functions such as primate alarm calls have low $J_{t}$ (individual observation of threat), high $b_{t}$ (obvious threat such as a predator), and exhibit Gaussian frequency distributions (Burling 1993; Ouattara et al. 2009).

\subsection{Relationships}

A specific category of language, names within traditional kin systems, acts as a proxy for relationships by informing people of how to behave toward one another. For traditional naming systems, the distribution of name popularity is constrained by the number of kin in different categories, but, of course, first names are socially learned from other kin. Good examples that use big data are the naming networks of Auckland, New Zealand, which Mateos et al. (2011) have visualized online (http://www. onomap.org/naming-networks/fig2.aspx). Mateos et al. also looked at 17 countries, finding that first-name popularity distributions retain distinct geographic, social, and ethnocultural patterning within clustered naming networks. Many traditional naming systems, therefore, belong in the northeast quadrant, after evolving over generations of cultural transmission into adaptive means of organizing social relations (Jones 2010).

If traditional naming tends to map in the northeast quadrant, then the exceptional freedom of naming in modern "WEIRD" (Western, Educated, Industrialized, Rich, and Democratic) nations (Henrich et al. 2010) fits the fashionable nature of the southeast model (Berger \& Le Mens 2009). Studying recent trends in Norwegian naming practices, Kessler et al. (2012, p. 1) suggest that "the rise and fall of a name reflect an 'infection' process with delay and memory." Figure 4 shows how the popularity of baby names yields a strikingly consistent, nearly power-law distribution over several orders of magnitude. Also expected for the southeast, the twentieth-century turnover in the top 100 U.S. names was consistent, with about four new boys' names and six new girls' names entering the respective top-100 charts per year (Bentley et al. 2007).

Although it should not be surprising if names traditionally map in the northeast, and have shifted southeast in Western popular culture, what about relationships themselves? For prehistoric hominins, one of the most basic resource-allocation decisions concerned the number of relationships an individual maintained, which was constrained by cognitive capacity and time allocation (Dunbar 1993). We would expect such crucial resourceallocation decisions to plot in the northwest, with low $J_{t}$ and high $b_{t}$. We therefore expect a Gaussian distribution of number of relationships, although the mean will vary depending on how costly the relationships are. Indeed, the "Dunbar number" (Dunbar 1992) limit of approximately 100-230 stable social relationships per person ${ }^{6}$ refers to a mean of this normal distribution, which varies according to relationship type (e.g., friendships, gift partners, acquaintances).

Figure 5 shows that the mean number of gift partners among hunter-gatherers is Gaussian, with a mean of a 
Bentley et al.: Mapping collective behavior
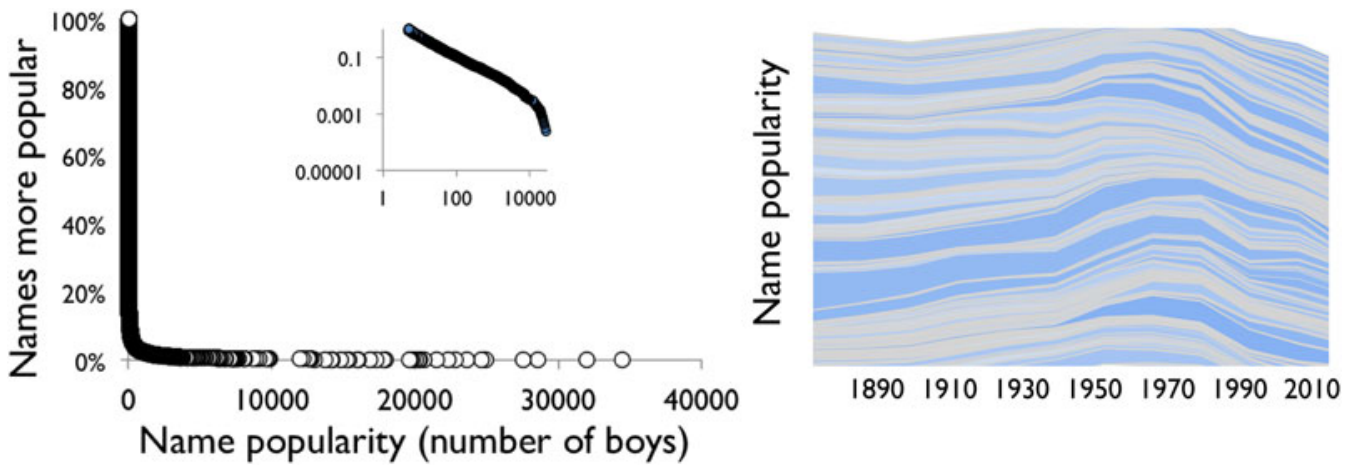

Figure 4. Distribution of the popularity of boys' names in the United States: left, for the year 2009; right, top 1,000 boys' names through the twentieth century, as visualized by babynamewizard.com.

few or several individuals (Apicella et al. 2012). The data are from surveys (subjects were asked to whom they would give a gift of honey) of more than 200 Hadza (Tanzania) women and men from 17 distinct camps that have fluid membership (Apicella et al. 2012). The Gaussian distribution in Hadza "gift network" size surely reflects the constraint of living in camps of only about 30 individuals. In the post-industrial West, with the differences of time expenditure, we might expect a larger mean number of relationships, but still with the same evidence for low $J_{t}$ and high $b_{t}$ of the northwest. Figure 5 shows how the distribution is similar to that of close friends among students at a U.S. junior high school (Amaral et al. 2000). We expect the distribution mean to increase even further as relationships go online. The mean number of Facebook friends per user (Lewis et al. 2008, 2011) is indeed larger, and yet still the distribution follows almost the same Gaussian form when scaled down for comparison (Fig. 5). This Gaussian distribution of friends per Facebook users is

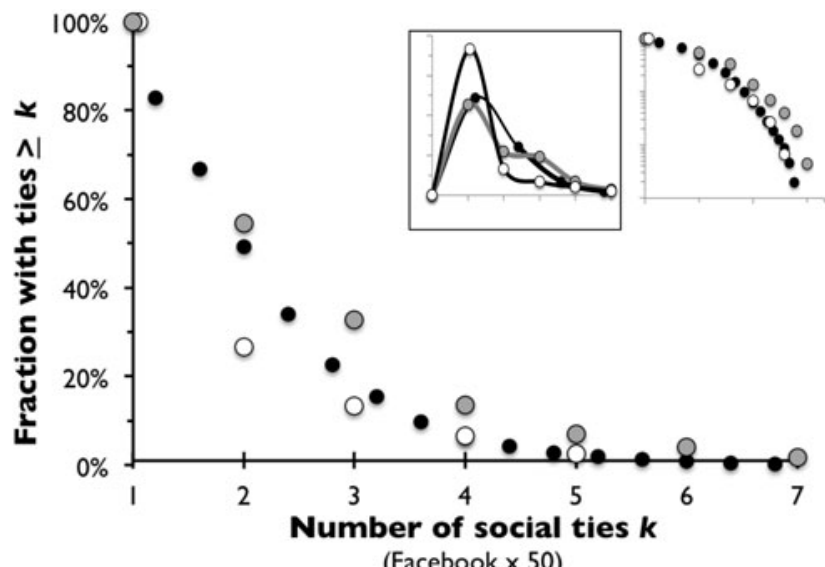

(Facebook $\times 50$ )

Figure 5. Gaussian distributions of social ties per person as examples of behavior in the northwest. Open circles show hunter-gatherer gift-exchange partners, gray circles show school friends, and black circles show Facebook friends. The main figure shows cumulative distributions of social ties per person; the unboxed inset shows the same distribution on double logarithmic axes (compare to Figure 2, northwest inset); and the boxed inset shows the probability distribution. Data for the hunter-gatherer gift-exchange partners are from Apicella et al. (2012); for school friends from Amaral et al. (2000); and for Facebook friends from Lewis et al. (2008; see also Lewis et al. 2011). consistent, with their interaction network being bounded at around 100 (Viswanath et al. 2009). Given that the friendship distributions are consistent for the different categories of relationships, we assume their Gaussian distributional form has been consistent through time, as expected for the northwest (Fig. 2b).

Unlike Facebook, other online social networks (e.g., Skitter, Flickr) show more fat-tailed distributions in numbers of relationships; these right-skewed distributions resemble information-sharing sites such as Digg, Slashdot, and Epinions (konect.uni-koblenz.de/plots/degree_distribution). Krugman (2012) points out how the number of followers among the top-100 Twitter personalities is long-tailed, and indeed the continually updated top 1,000 (on twitaholic.com) is log-normally distributed, as confirmed by a massive study of over 54 million Twitter users (Cha et al. 2012). Checking in June 2012 and then again in the following November, we found that the log-normal distribution of followers remained nearly identical (in normalized form), despite Twitter growing by roughly 150,000 followers a day.

These heavily right-skewed distributions place Twitter in the east, with high $J_{t}$, but is it northeast or southeast? Overall, the turnover and right-skewed popularity distributions of Twitter would appear to map it in the southeast. By tracking the most influential Twitter users for several months, Cha and colleagues (2010) found that the mean influence (measured through re-tweets) among the top 10 exhibited much larger variability in popularity than the top 200. This can be roughly confirmed on twitaholic. com, where the mean time on the top-1,000 Twitter list on June 10, 2012, was 42 weeks, with no significant correlation between weeks on the chart and number of followers. Twenty-four weeks later, the mean time on the top-1,000 list had increased by six weeks. At both times, the lifespan in the top 10 was only several weeks longer than that in the top 1,000 , suggesting surprisingly little celebrity (prestige) bias as opposed to just unbiased copying that occurs in rough proportion to current popularity.

These changes appear to reflect the media more than the individuals within them. Twitter, for example, is more a broadcasting medium than a friendship network (Cha et al. 2012). Unlike with traditional prestige, popularity alone on Twitter reveals little about the influence of a user. Cha and colleagues found that Twitter allows information to flow in any direction (southeast) rather than the majority learning from a selected group of well-connected "influentials" (northeast). Because information 
flows in all directions, we place Twitter in the southeast, as it "does not follow the traditional top-to-bottom broadcast pattern where news content usually spreads from mass media down to grassroots users" (Cha et al. 2012, p. 996). This suggests information sharing may be shifting southeast even if actual relationships are not. Indeed, there is evidence for a critical level of popularity, where the downloading of "apps" from Facebook pages shifts eastward on our map, that is, from individual decision making to socially based decision making (Onnela \& Reed-Tsochas 2010).

\subsection{Wealth and prestige}

Although Twitter exhibits a new, grassroots role in terms of influencing and spreading information, there is no doubt that just about all the members of the top-1,000 on Twitter are prestigious, at least by the definition of Henrich and Gil-White (2001), in that millions of people have freely chosen to "follow" them. If prestige and popularity are to become synonymous on Twitter, then prestige on this medium will plot in the southeast, as people naturally copy those whom others find prestigious rather than make that determination individually (Henrich \& GilWhite 2001). To an unprecedented level, Twitter clearly exploits our preference for "popular" people, which "evolved to improve the quality of information acquired via cultural transmission" (Henrich \& Gil-White 2001, p. 165). Twitter celebrities are thus more able to act as "evangelists" who can "spread news in terms of . . . bridging grassroots who otherwise are not connected" (Cha et al. 2012, p. 997).

Most of the top-1,000 Twitter personalities are also wealthy, of course, and prestige, wealth, and popularity are well entwined in this medium. Could this signal a future shift toward the southeast? We would normally map gift-giving in the northeast, as a transparent means of maintaining relationships and reducing risk through social capital (Aldrich 2012). Even in the West, patterns of charity still exhibit northeast patterns. Charitable giving in the United States from 1967 to the present, for example, has exhibited a log-normal distribution per category (religion, education, health, arts/culture, and so on) that did not change appreciably in form over a 40 -year period (Fig. 6). Nor did the rank order of charitable giving by category change. It is immediately striking what a long-term, sustained tradition charitable giving is among generations of Americans.

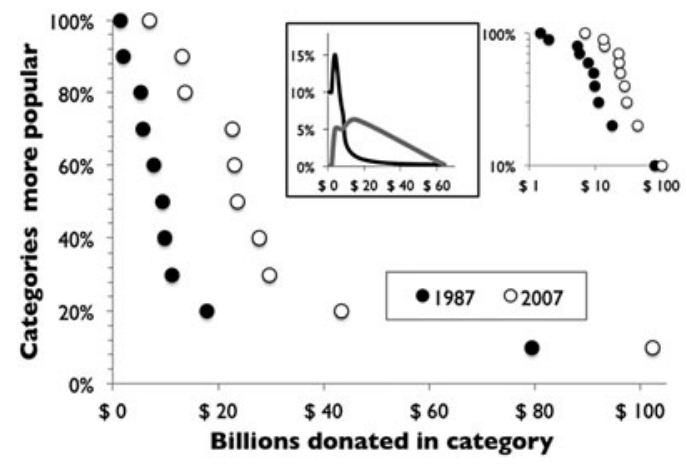

As charitable-giving habits are inherited through the generations, the wealth of the recipients, such as universities and churches, depends on this northeast pattern. It should not be surprising, then, if wealth itself shows a northeast phenomenon, even in small-scale societies. Pareto distributions of wealth typify modern market economies, but even in traditional pastoralist communities, for example, wealth may follow the highly right-skewed form of the northeast. Figure 7 shows how distributions can vary. We would map Karomojong pastoralists (DysonHudson 1966), whose cattle-ownership distribution is the most Gaussian (Fig. 7), farther west than the Ariaal of northern Kenya (Fratkin 1989) and the Somali (Lewis 1961), whose wealth is highly right-skewed (Fig. 7). This fits with ethnographic information, as Karomojong pastoralists culturally impose an equality among members of each age set (Dyson-Hudson 1966), whereas Ariaal herd owners "increase their labor through polygyny, increased household size, and the hiring of poor relatives" (Fratkin 1989, p. 46). Transparency of wealth increases with the rising cost of competition and the agglomeration of power, as powerful tribes expand at the expense of smaller groups (Salzman 1999).

Given these northeast patterns for the fairly static distributions of wealth and prestige in traditional societies, what

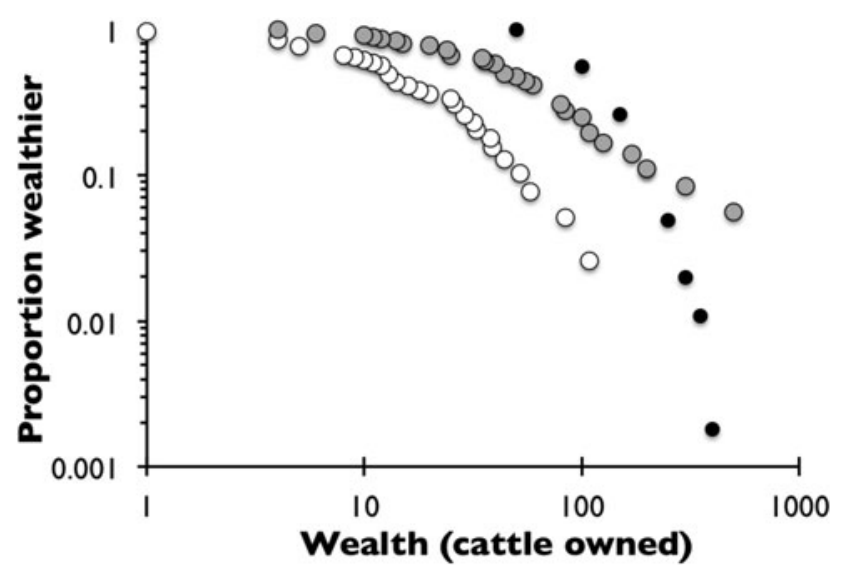

Figure 7. Wealth among pastorialists, with a cumulative probability distribution plot comparing published ethnographic data from the Somali (Lewis 1961) with gray circles, the Ariaal (Fratkin 1989) with white circles, and Karomojong (DysonHudson 1966) with filled black circles.

Figure 6. Charitable giving in the United States: left, past 30 years, at the national level; right, relative growth of several categories of charitable giving in the United States, over a 40-year period (popularity is expressed as the fraction of the total giving for that year, on a logarithmic scale). Data from Giving USA Foundation (2007). 
could we expect from emerging southeast patterns in an online society? On the one hand, we have a pull toward the southeast in highly social realms of information overload such as Twitter. On the other hand, powerful search engines that help people find the most relevant information from others, the ubiquity of social rating, and the increased relevance of "grassroots" influences (Cha et al. 2012) may keep things in the northeast, as social learning is better informed. If intelligent search technology gains the upper hand on information overload, then $b_{t}$ increases and the crucial variable is $J_{t}$. If $J_{t}$ is low, then prestige or exclusive access to resources is less transferrable from one person to another, and inequality is lessened.

\section{Discussion and conclusions}

To address collective behavior in the big-data era, the map we present here, with its dimensions of social influence and transparency of payoff, has allowed us to situate big data in a much broader perspective, alongside data from past and present societies. Although some of the conclusions we make from these data are clear from qualitative observation, the new world of big-data research makes it impossible to personally witness all of the decisions being made. We therefore have sought a set of empirical signatures that can be detected in massive sets by simple statistical means, which we might someday even see automated. The mapping then helps identify the more granular tools of various social-science research traditions that will be most useful for a particular case study: the northwest (economic), the southeast (cultural-historical), the northeast (both), and the southwest (neither).

The map quadrants can be characterized by different patterns - change through time and distributions of popularity - that can be gleaned from the kinds of data that the behavioral sciences hope to understand. The map is more than an academic exercise. It becomes highly practical with respect to public policy, for example, by providing direction over whether it is more effective to disseminate information in the northwest, engage targeted "word of mouth" campaigns in the northeast, or place many bets more randomly in the southeast (Bentley et al. 2011; Sela \& Berger 2012; Watts \& Hasker 2006). The map provides a means for evaluating population-level trends in the kinds of decision-making categories noted earlier-voting, opinions on climate change, mating, health care, consumer trends - which we see beginning to move to the southeast.

This can be important in a world where policy may not match the way human decisions are made. Much of traditional social science and policy has used the northwest as its base assumption that public behavior is dictated by cost/benefit ratios and incentives, even if slightly flawed or biased in perception (e.g., Kahneman 2003). This comprehensive practicality is why the map is not just another theoretical classification scheme. It allows a data-driven study question, reframed as a hypothesis, to be tested with appropriate datasets by examining whether $b_{t}$, the degree of transparency of payoffs, decreases through time and whether $J_{t}$, the intensity of social influence, increases through time for contexts in which the $\left(b_{t}, J_{t}\right)$ discretechoice framework is appropriate. Because these dimensions are general, realms of behavior can potentially be linked through this approach. For example, the discretechoice framework has been used to document the rise of polarized political behavior in the United States: Li and Lee (2009) found $J_{t}>0$ for the Clinton-Dole presidential election, and McCarty et al. (2006) linked this polarization to the dramatic rise in income inequality.

Of course, $b_{t}$ and $J_{t}$ are not everything. One interesting future project would be to explore the effects of other dimensions (parameters) that we have deliberately avoided with our simplified map. Where social learning is well informed, personal biases such as trust, prestige, and status will obviously matter (e.g., Eriksson et al. 2007; 2010; Henrich \& Gil-White 2001). Perhaps the most important phenomenon we might next consider is how the structure of social networks affects the dynamics of social learning (e.g., Borgatti et al. 2009; Dodds \& Watts 2005). The map quadrants relate to contemporary debates such as the "spread" of obesity (Christakis \& Fowler 2007) or voting and consumer preferences (Aral \& Walker 2012), in which each debate revolves around whether certain behaviors are really spreading along a directed network as opposed to like-minded people simply associating (e.g., Shalizi \& Thomas 2010). The map changes the discussion about interaction networks. Rather than a "wiring diagram" between human beings, what matters is understanding how the dynamics of decision making derive from overall network structure (Ormerod 2012). For example, highly clustered social networks appear to favor the spread of norms of cooperation (Ohtsuki et al. 2006) and norms of innovations by introducing them repeatedly to individuals through different neighbors of a cluster (Centola 2010; Helbing \& Yu 2009; Lorenz et al. 2011).

More generally, Lieberman et al. (2005) identified those social-network arrangements favorable to selection (northeast) versus random drift, or undirected copying (southeast), in the sorting of variation. These generalized networks fit the map well. In Figure 8, we have placed in the southeast the diffuse networks that Lieberman et al. (2005) identify with drift and in the northeast the hierarchical networks they associate with selection. Figure 8

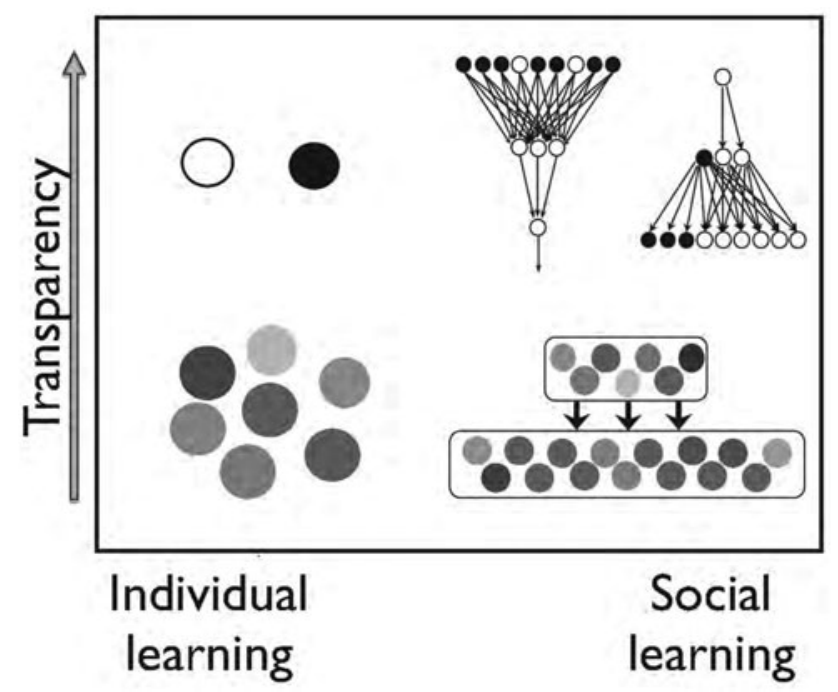

Figure 8. A representation of how social-network structure (or lack thereof) fits onto the map (schema adapted from Lieberman et al. 2005). In each quadrant, circles represent agents, and shades represent their choices. In the east, arrows represent social influences through which agents make most of their choices, whereas in the west, agents make choices independently. 
illustrates how the map organizes the behavior of a binary dynamic-choice system. The northwest quadrant illustrates that when the intensity of choice, $b_{t}$, is very large, $P_{+, t}$ will be close to one when the net payoff to +1 is positive (the dot is solid black) and will be close to zero when the net payoff to +1 is negative (the dot is solid white). This case corresponds to a very strong force of selection in Krakauer's (2011) evolutionary dynamics, meaning that the replicator equation has a large value of $b_{t}$. The mix of dots with various shades of gray in the southwest depicts the wide range of values of $P_{+, t}$ when $b_{t}$ is very small, even when the net payoff to +1 is positive. The directed networks (with either black dots or white dots for the choices) depicted in the northeast quadrant are intended to capture the idea that social influence will be very strong (and $P_{+, t}$ will be close to one or zero) when $b_{t}$ is large, even if $J_{t}$ is of moderate size. The various shades of gray in the directed network in the southeast quadrant are intended to capture the idea that many values of $P_{+, t}$ are likely to be observed because $b$ is small.

The network rendition of the map helps us match bigdata patterns to practical or theoretical challenges. If one aims to shift collective behavior from the herdlike southeast to the more-informed northeast, then learning networks ought to become more directed and hierarchical. In fact, it appears that hierarchical networks are a natural component of group adaptation (Hamilton et al. 2007; Hill et al. 2008; Saavedra et al. 2009) and may provide a crucial element to the debate over the evolution of cooperation, for which punishment of non-cooperators appears to be insufficient (Dreber et al. 2008; Helbing \& Yu 2009; Rand et al. 2009).

In this way, the map puts big data into the big picture by providing a means of representing, through case-specific datasets, the essence of change in human decision making through time. In a given context, we may wish to assess, from population-scale data, whether people are still responding to incentives and/or behaving in nearoptimal ways with respect to their environment (e.g., Horton et al. 2011; Nettle 2009; Winterhalder \& Smith 2000). If so, then cumulative innovations should generally improve technological adaptation over time or even human biological fitness (Laland et al. 2010; Milot et al. 2011; Powell et al. 2009; Tomasello et al. 1993). Conversely, if the vicissitudes of social transmission, fads, and cultural drift are driving innovation, then such improvements are not guaranteed (e.g., Cavalli-Sforza \& Feldman 1981; Koerper \& Stickel 1980).

A big question is whether change in hominin evolution has been roughly clockwise, from the individual learning of the northwest, to the group traditions of the northeast with the evolution of the social brain, to the south - particularly the southeast - as information and interconnectedpopulation sizes have increased exponentially through time (Beinhocker 2006; Bettencourt, Lobo \& Strumsky 2007). If we consider the smaller societies of human prehistory, crucial resource-allocation decisions would plot in the northern half of the map, and the more specific behaviors would range between the northwest and the northeast. Because Homo economicus is located at the extreme northwest, he does not appear to be the primary model for human culture, considering that the deep evolutionary roots of human sociality, social-learning experiments, and ethnographic research all suggest that social learning is how small-scale societies have adapted to environments for most of human existence (e.g., Apicella et al. 2012; Byrne \& Russon 1998; Henrich et al. 2001, 2005; Tomasello et al. 2005). The ideal position in the northeast appears to be dictated by the spatial and temporal autocorrelation of the environment and the cost of individual learning (Mesoudi 2008).

The examples we discussed in section 3 place many social-network media on the east side of the map. This does not necessarily mean that people are fundamentally changing, however, although perhaps they are to a small degree (Sparrow et al. 2011). Rather, it means that online environs sort behavior differently. In our Internet world of viral re-tweets and their associated scandals, interest in a "world brain" - a science fiction of H. G. Wells's - has been reborn, as the number of people exchanging ideas in an economy determines the complexity of a nation's science and technology (Hausmann et al. 2011).

When humans are overloaded with choices, they tend to copy others and follow trends, especially apparently successful trends. If too far southeast, this corrodes the distributed mind. One realm where this may clearly be important is academic publishing. Should we worry that academic publishing may drift southeast? A drift in this direction certainly seems possible, especially with quality no longer transparent among an overwhelming number of academic articles (Belefant-Miller \& King 2001; Evans \& Foster 2011; Simkin \& Roychowdhury 2003). To maintain transparency in the northeast, it seems sensible to maintain support for rigorous, specialist-access academic journals, against pressure to blur scientific publications with blogs and social media (Bentley \& O'Brien 2012).

Could a drift southward be even a more-general trend? Successful technological innovations generate a multitude of similar options, thereby reducing the transparency of options and payoffs (O’Brien \& Bentley 2011). One consequence of this proliferation of similar alternatives is that it becomes more and more difficult for learning processes to discover which options are in fact marginally better than others. This pushes decisions southward and paradoxically may mean that modern diverse consumer economies may be less-efficient crucibles for the winnowing of life-improving technologies and medicines than societies were in the past and some traditional societies are today (Alves \& Rosa 2007; Marshall 2000; Voeks 1996). It might be argued that the drift in mass culture toward the southeast is not a particularly fit strategy, as the propensity for adaptation found in the north is lost.

We might assume that because we've spent most of our evolutionary history in the north, the "best" behaviors, in terms of fitness, are the ones that become the most popular. It could be argued that important health decisions ought to lie in the northwest, and in traditional societies it seems that's the case-such decisions are not strongly socially influenced (Alvergne et al. 2011; Mace \& Colleran 2009). To the extent that there is social influence, it typically comes from close kin (Borgatti et al. 2009; Kikumbih et al. 2005). As modern mass communication becomes available, however, the cost of gaining health information socially declines - a shift eastward on the map - but it also makes socially transmitted health panics more common (Bentley \& Ormerod 2010).

In conclusion, we note that it is easy to be intimidated by big-data studies because the term really means BIG data. 
In these early days of big data, however, many studies seem to show us the obvious. In the best case, big-data studies will not compete with more traditional behavioral science but instead will allow us to see better how known behavioral patterns apply in novel contexts. In fact, they may even validate the most basic Bayesian analysis of human behavior there is, which is human experience. Humans sample the actions of their peers just by living among them for a lifetime. This takes us back to the northwest: Popularity does not guarantee quality. As long as people trust their own individual experiences, even in observing the behavior of others, a collective wisdom is possible.

\section{ACKNOWLEDGMENTS}

We thank Barbara Finlay, Herbert Gintis, David Geary, and our anonymous reviewers for excellent comments and advice on earlier drafts. We also thank Daniel Nettle for his many insights and suggestions for strengthening the argument, and Jonathan Geffner and Sumitra Mukerji for their editorial and production assistance.

\section{NOTES}

1. We can be more precise in the context of empirical statistical work by specifying $\left(b_{t}, J_{t}\right)$ as functions of covariates and parameters of interest to estimate - for example, $b\left(x_{t}, \theta_{b}\right), J_{t}=J\left(x_{t}\right.$, $\theta_{J}$ ), where $x_{t}$ is a vector of potentially relevant covariates, which can include past values of the same covariates as well as past average choices over potentially relevant reference groups (for potential "contagion" effects) and average choices over potentially relevant reference groups (for potential "contextual" effects) (Manski 1993). Here, $\theta_{b}$ and $\theta_{J}$ are vectors of parameters that can be estimated. Once estimation is done, hypotheses can be proposed and tested using statistical methods. The era of "big data" opens up new possibilities for empirical work, formulation of hypotheses, and formal statistical testing of these hypotheses versus plausible alternatives.

2. The intense interest in these distributions, such as power laws, has led to a productive debate such that multiple alternative right-skewed distributions are now critically compared, with recognition that subtle differences in distributions can be informative as to the processes that produce them (Frank 2009; Laherrère \& Sornette 1998; Venditti et al. 2010).

3. Care must be taken with the assumption that patterns in the northwest will always be Gaussian. Here is an example to the contrary. Consider the discrete-choice model with two choices $\{-1$, $+1\}$ and with $b_{t}$ and $J_{t}$ being anywhere from zero to infinity. Let $h_{t}=u_{+, t}-u_{-, t}$, which is just the payoff difference of the two options. Then, the probabilities of choice at date $t$ are given by

$$
P_{+, t}=\frac{\mathrm{e}^{b_{t} h_{t}}}{1+\mathrm{e}^{b_{t} h_{t}}}, \quad P_{-, t}=\frac{1}{1+\mathrm{e}^{b_{t} h_{t}}} .
$$

Suppose that $h_{t}$ exhibits a Gaussian distribution with zero mean and finite variance. As $b_{t}$ approaches infinity, we observe only $P_{+, t}=0$ or 1 . Here we see that a Gaussian distribution of $h_{t}$ is turned into a bimodal distribution with all mass at 0 or 1 , even though we are in the northwest quadrant of the map. In the southwest, $b_{t}$ is small. At the extreme south it is zero, and $P_{+, t}=1 / 2$, $P_{-, t}=1 / 2$, no matter the value of $h_{t}$. Now, given that $h_{t}$ exhibits a Gaussian distribution with mean zero and finite variance, we can see that a small value of $b_{t}$ will produce a unimodal, hence "Gaussian looking," distribution of $P_{+}$when the system is in the southwest quadrant. As we move north by increasing $b_{t}$, we expect eventual bimodality of the distribution of choice probabilities, $\left\{P_{+, t}\right\}$, as we sample this choice process over time with increasing $b_{t}$.

4. One established model assumes the popularity, $n_{t}$, of a choice at time $t$ as proportional to its popularity at time $t-1$ :

$$
n_{t}=\left(1+g_{t}\right) n_{t-1}
$$

where $g_{t}$, normally distributed over time, expresses the fluctuating rate at which agents in the population make new decisions. The result is a log-normal distribution of the accumulated popularity that spreads outward through time on a logarithmic scale. The model holds that the probability of a behavioral option accumulating popularity $n$ at time $t$ is given by

$$
P(n)=\frac{1}{n \sqrt{t 2 \pi \sigma^{2}}} \exp \left\lfloor-\frac{\left(\ln n-g_{0} t\right)^{2}}{2 \sigma^{2} t}\right\rfloor,
$$

where $g_{0}$ is the mean of $g$ over time, with standard deviation $\sigma$. The position $g_{0} t$ and width $\sigma^{2} t$ of the log-normal peak increases with time $t$, such that the accumulated popularity distribution for options of the same age (e.g., citations of journal articles published in the same year) spreads outward through time on a logarithmic scale. This model can also be fit dynamically, as described by $\mathrm{Wu}$ and Huberman (2007): For each behavioral choice at time $t$, one calculates the logarithm of its popularity minus the logarithm of its initial popularity when the sampling started. Then to represent time $t$, the mean versus the variance of these logged values is plotted. Repeating this for all time slices in the sample, the resulting cluster of points will yield a linear correlation between the means and variances of the logged values (i.e., for this area of the northeast quadrant).

5. In the negative-binomial theorem, the probability of $k$ choices of specific option $x$, given that there have been $k+r$ total choices overall, is as follows:

$$
\operatorname{Pr}(x=k)=\left(\begin{array}{c}
k+r-1 \\
k
\end{array}\right) p^{k}(1-p)^{r}
$$

6. A group size of 150 often is quoted as an average, but Dunbar never used either an average or a range in his original paper (Dunbar 1992), which had to do with neocortex size and group size in nonhuman primates. Group size in humans was addressed in later papers (Dunbar 1993; 1998). Often misunderstood is that Dunbar was referring to "meaningful" relationships, not simply the number of people one remembers: "The social brain hypothesis is about the ability to manipulate information, not simply to remember it" (Dunbar 1998, p. 184).

\section{Open Peer Commentary}

\section{"Big data" needs an analysis of decision processes}

doi:10.1017/S0140525X13001659

\section{Pantelis P. Analytis, ${ }^{a}$ Mehdi Moussaïd, ${ }^{a}$ Florian Artinger, ${ }^{b}$ Juliane E. Kämmer, ${ }^{a}$ and Gerd Gigerenzer ${ }^{a}$ \\ ${ }^{a}$ Adaptive Behavior and Cognition, Max Planck Institute for Human Development, 14195 Berlin, Germany; ${ }^{\mathrm{b} B e h a v i o u r a l ~ S c i e n c e ~ G r o u p, ~}$ Warwick Business School, The University of Warwick, Coventry CV4 7AL, United Kingdom. \\ analytis@mpib-berlin.mpg.de moussaid@mpib-berlin.mpg.de florian.artinger@wbs.ac.uk kaemmer@mpib-berlin.mpg.de gigerenzer@mpib-berlin.mpg.de http://www.mehdimoussaid.com/}

Abstract: We demonstrate by means of a simulation that the conceptual map presented by Bentley et al. is incomplete without taking into account people's decision processes. Within the same environment, two decision processes can generate strikingly different collective behavior; in two environments that fundamentally differ in transparency, a single process gives rise to virtually identical behavior. 
We applaud Bentley et al. for postulating a map of the environment in the form of a two-by-two classification. Too much of psychological theorizing still focuses on internal factors alone, such as traits, preferences, and mental systems - a kind of theorizing that social psychologists once labeled the "fundamental attribution error." Yet Bentley et al. are in danger of committing the opposite error: to theorize without regard of the cognitive processes. Herbert Simon (1956) noted more than 50 years ago that decision making is rather akin to the two blades of a scissors: the one blade is the decision strategy or heuristic; the other, the environment. Decision strategies have evolved and adapted to a given environment, and their rationality is ecological: therefore, one needs to analyze both the cognitive processes and the structure of the environment (Gigerenzer et al. 1999).

To apply this argument to Bentley et al., we show that the distributions in the four quadrants do not simply depend on the two environmental features, but, in addition, on the decision processes people rely on. We illustrate this point by a demonstration: We define two decision processes that have a strong social component but differ in whether social influence comes in the first step (the construction of the consideration set) or in the second step (the choice from this set). We then demonstrate that (1) within the same environmental structure (quadrant), the two decision processes can generate different distributions, and that (2) when the same decision process is used in two quadrants that differ in terms of transparency, the resulting distributions are almost identical.

We simulate 10,000 agents who sequentially choose from a set of 100 items (e.g., cameras, wines) drawn from a multivariate normal distribution. The agents aim for the item with the highest possible quality. The agents cannot evaluate the quality of the item with certainty but have to infer it from three attributes. They learn about each attribute's validity, that is, the relative frequency with which the attribute correctly predicts the item with the highest quality in pair-comparisons, in an initial training phase where they randomly sample 10 items.

Endowed with this knowledge, agents use a two-step decision process to select the best item. In the first step, each agent forms a subset of the available items, the consideration set $(n=3$; Hauser \& Wernerfelt 1990). In the second step, the agents decide which item to choose from this set by using a lexicographic decision rule akin to the take-the-best heuristic (Gigerenzer \& Goldstein 1996). It ranks the attributes according to their validity; the item with the highest value on the most valid attribute is then chosen. In case two or more items have the same value on the first attribute, the second, and then the third attribute, respectively, is examined; if neither discriminates, the agents choose randomly.

Social influence is introduced in both processes but in different steps of the process. In the popularity-set heuristic, social influence is introduced in the first step. The probability that an item is part of the consideration set is proportional to how often the item has been selected by other agents in the past. In the popularity-cue heuristic, social influence enters in the second step as an additional, equally treated, fourth attribute corresponding to how often the item had been selected by others.

In the low-transparency environment, the correlations between quality and the three attributes are weak $(0.11,0.10,0.10)$. In the high-transparency environment, the correlations between quality and attributes are strong $(0.90,0.69,0.61)$. These environments correspond to the very north and south of the map by Bentley et al. just short of the border. In both scenarios, the inter-correlations between the attributes are held constant at 0.4. [The details of the simulation can be found at: http://www.mehdimous said.com/.]

As shown in Figure 1, the popularity-set heuristic generates collective herding, regardless of the environmental features. Here, a feedback loop operates: The more people choose an item, the more this item becomes attractive for subsequent decision-makers.
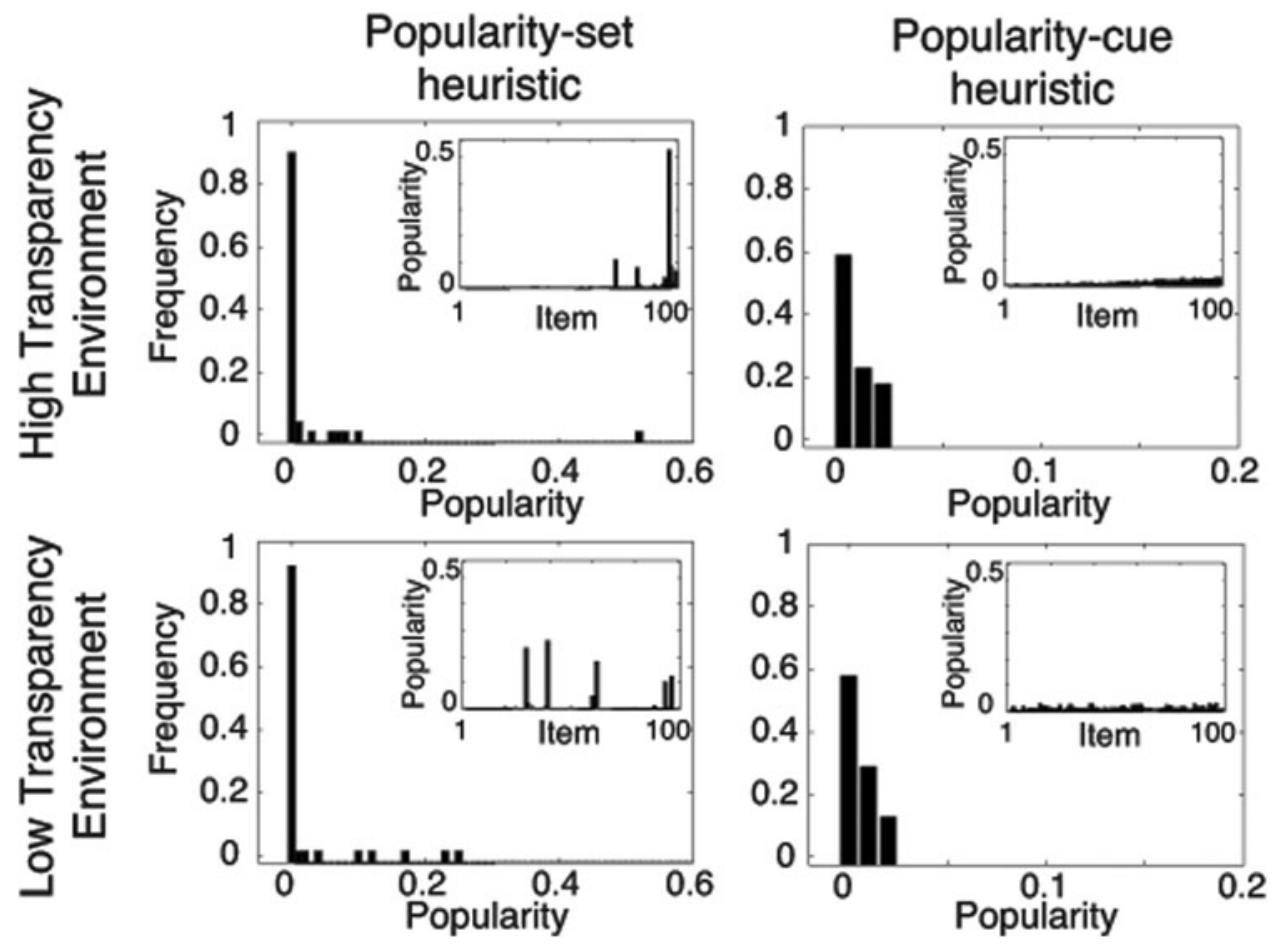

Figure 1 (Analytis et al.). The popularity distributions do not simply depend on Bentley et al.'s distinction between low- and hightransparency environments but also on the decision processes (heuristics). Within the same environmental structure (rows), the distributions change with the decision processes. When the same process (heuristic) is applied to different environments (columns), the result can be the same distribution. The outside graphs show the distribution of items' popularity at the end of the simulation. The popularity of an item is measured as the fraction of agents who have chosen that item. The inner graphs show the popularity reached by each of the 100 items separately, which are ordered according to their quality, from low quality (left) to high quality (right). 
This leads to a few products becoming very popular, while most others are ignored. In contrast, the popularity-cue heuristic generates a homogeneous distribution of popularity, where each item receives a similar, low amount of choices, in high- and lowtransparency environments alike. Here, social influence applies to a reduced subset of the items only, which prevents the feedback loop from setting up. These results show that different processes generate strikingly different results in the same environment. Likewise, the same process generates similar results when it is applied to different environments.

The conceptual map by Bentley et al. neatly reduces the environment to two variables. However, whether decisions are arrived at independently or not, and whether the information is transparent or not, is only half of the story. To fully understand what patterns emerge, one needs to account for the decision process. The relevance of such an approach is heightened by the interdependence of the social context where both mind and environment adapt to each other (see, e.g., Artinger \& Gigerenzer, in preparation; Moussaïd et al., 2013). The advent of big data and the combination of experimental and simulation methods provide ample opportunities to study adaptive decision processes, stepping outside the black box of "as-if" decision theories.

\section{"The map is not the territory"}

\author{
doi:10.1017/S0140525X13001660
}

\section{Fred L. Bookstein}

Department of Anthropology, University of Vienna, A-1091 Vienna, Austria, and Department of Statistics, University of Washington, Seattle, WA 98195. fred.bookstein@univie.ac.at

\begin{abstract}
Bentley et al.'s claim that their "map ... captures the essence of decision making" (target article, Abstract) is deconstructed and shown to originate in a serious misunderstanding of the role of principal components and statistical graphics in the generation of pattern claims and hypotheses from profile data. Three alternative maps are offered, each with its radiation of further investigations.
\end{abstract}

My title, "The map is not the territory," is a famous sentence from a 1931 lecture by the general semanticist Alfred Korzybski (1933, p. 750). His meaning is that the graphical structure of a map need not be the structure of the territory (here, the scientific field) that it purports to represent. Statistical graphics, the discipline to which I have migrated his aperçu, is a field in which this insight has particular force. The target article's authors, Bentley et al., declare in their Abstract that they have "create[d] a multiscale comparative 'map' that, like a principal-components representation, captures the essence of decision making," that "each quadrant ... features a signature behavioral pattern," and that "the map will lead to many new testable hypotheses concerning human behavior." My critique would have been Korzybski's: this map of theirs is not the territory, and cannot be trusted to capture any "essence." In particular, its topology, which is the conventional topology of principal component score plots, is all wrong for this subject-matter, which is preference profile patterns.

The objection is not so much to Bentley et al.'s quadrants per se as to the geometry of their diagram, which, in keeping with the conventional interpretation of principal components, is flat with a particularly depauperate connectivity. In the upper left panel of my Figure 1, I have redrawn Bentley et al.'s Figure 1 to emphasize the contrasts that concern the authors; these are along the edges of this square. The corresponding philosophical anthropology is a pure distillation of Levi-Strauss: two verbal oppositions ("independent" vs. "social" and "transparent" vs. "opaque") are treated as if these are as obvious and fundamental as water versus land, male versus female, light versus darkness, alive

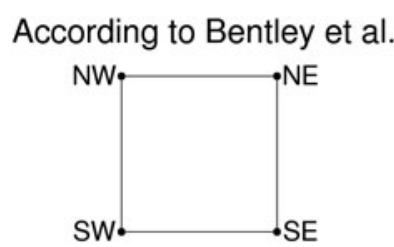

NW vs SW, SW vs SE,
SE vs NE, NE vs NW'
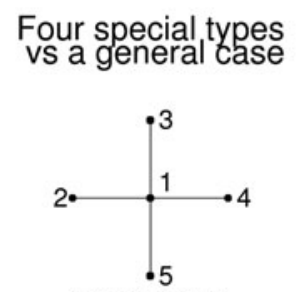

1 vs 2,1 vs 3

1 vs 4,1 vs 5
The tetrahedron (all pairs)

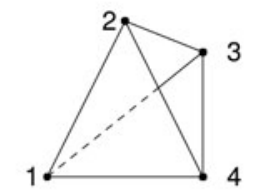

1 vs 2,2 vs 3,3 vs 4

2 vs 3,2 vs 4,3 vs 4

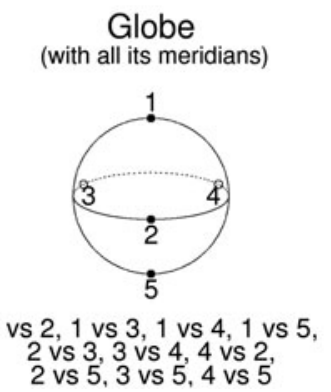

Figure 1 (Bookstein). The "map" according to Figure 1 of Bentley et al., together with three other graphics (tetrahedron, contrast with a general type, globe) based on the same data resources but leading to quite different lists of contrasts, rhetorics of interpretation, and suggested hypotheses for subsequent testing. See text.

versus dead, or raw versus cooked, then converted into abstract propositions. We have no information about relationships along the diagonals, or relationships of the periphery to the center; in fact, the center is no construct at all. Nor is there any argument that the right axes are the north-south and east-west here; the phrases remain mere words. Nor do we know if the meaning of "independent versus social," for instance, is the same in "transparent" as in "opaque" domains of decision-making. There may be more than two types of edges here.

"The map," which is Bentley et al.'s Figure 1, is certainly not "the territory," which is the actual information content of the data resources. For data arising from samples of time-series, as shown in Bentley et al.'s Equation (1) and Figures 2 and 3, there are many other ways to organize a diagram that lead to quite different reporting languages and quite different "testable hypotheses." Here are three other possibilities.

Upper right: Four types symmetrically connected. This is the general situation of four types. No evidence is given that the configuration of the authors' data reduces to the two dimensions Bentley et al. show or, indeed, any two dimensions. Then there need to be six contrasts, not four.

Lower left: Four specialized types out of a common center. This is a commonly encountered topology in studies of biological evolution, wherein multiple descendant species are characterized by derived features that all descend from the same original feature. To the extent that preferences are developmental, they may embody the same central focus.

Lower right: Globe with an axis. Imagine Bentley et al.'s map as a local expansion of coordinate possibilities along an axis that shrinks these possibilities toward zero at either of two extremes, "everything popular" (without further profile) and "everything unpopular" (without further profile). The topology is now a globe. On it, the north and south poles stand for the pure configurations, while an equatorial band offers space for additional parameters corresponding to the decision profiles that incorporate behavioral modifiers. Such data structures are commonly encountered in the compositional sciences, such as mineralogy or personality profiles. The authors' map is the equatorial plane of this construction (but it still has the wrong topology). 
No one of these topologies is more consistent with the authors' data than any of the others, and nothing about their fundamental Equation (1) prefers the organization of a "map" over any other organization. Instead, each chart generates a different rhetoric of "testable hypotheses." For instance, the center-periphery topology at lower left would generate hypotheses that each "quadrant" defines itself with respect to an undifferentiated stem, not its opposite on either of the two proferred dimensions; while the topology of the globe would suggest that social agents place their profiles in a context of two extreme pure types, rather than profiling them after the stereotyped fashion of the "principal-components representation" mentioned in the target article's Abstract. The tetrahedral network in my Figure 1's upper right is a far more congenial context than the quadrant scheme for discussions of how a population might be "drifting," as drifting could be along any angle in the "map," not just the two cardinal directions.

If my argument here, which is essentially an appeal to foundations of graphical semiotics, is accepted, then the range of "testable hypotheses" flowing from Bentley et al.'s "big data" has been crippled by their choice of a stereotypical graphical metaphor, the "map," which is flat, with squares on it, and which profoundly lacks the options that real actors in a real situation will refer to as they go about their preference-linked business (e.g., reference profiles, satisficing criteria). It is unfortunate that the authors limit their scientific hypotheses to just the ones that flow from their unquestioned conventional graphical metaphor. For a social world in which the idealization of types need not correspond to any idealization either of their relationships or their representations in the minds of the agent, the imposition of a false symmetry of this kind entails the embrace of a potentially fatal rhetorical risk without any concomitant benefit.

\section{Extending the global village: Emotional communication in the online age}

\section{doi:10.1017/S0140525X13001684}

\section{Ross Buck}

Department of Communication, U-1259, University of Connecticut, Storrs, CT 06269 .

ross.buck@uconn.edu

http://coms.uconn.edu/directory/faculty/rbuck/index.htm

\begin{abstract}
Bentley et al.'s analysis of how human decision-making has changed in the online age does not mention emotion. Although the suggested dimensions involving social influence and transparency are interesting and suggestive, the engines behind the changes wrought by online media are arguably largely emotional, and implications of the communication of specific emotions via online media need to be better understood.
\end{abstract}

Bentley et al. present a significant, novel, and integrative approach to the understanding of collective decision-making, basing their analysis on two dimensions: the extent to which a decision is made independently versus socially; and the transparency versus opaqueness of payoffs and risks associated with the decision. Their analysis results in a fourfold map that, in their words, seeks to capture the essence of decision-making. Emotion is not mentioned in the target article, reflecting the long-standing dismissal of its effects on decision-making as relatively unimportant, largely disruptive, and contrary to rational thought. However, it is now widely understood that emotions can contribute positively to decisionmaking (Buck \& Ferrer 2012), and it is important to understand the conditions under which emotions can be facilitative or disruptive. Unfortunately, the conceptualization of emotion in the decision literature tends to be oversimplified, involving only positive-negative valence. Thus, experiences of risk described in the "risk as feelings hypothesis" are framed simply in terms of anticipated positive or negative evaluative feelings (Loewenstein et al. 2001).
I suggest that a more comprehensive understanding of the role of emotion in decision-making is possible if the Bentley et al. target article's "transparency-opaqueness" dimension is modified from a purely cognitive dimension involving the judgment of risk and reward to a dimension involving the interaction of reason and emotion (see Figure 1). The extreme left end of this dimension would involve emotion to the exclusion of reasonpure irrational passion - and would correspond to extreme opaqueness of payoffs and risks in the Bentley et al. analysis. To the right, the relative influence of reason grows until it is overwhelmingly important relative to emotion. However, the influence of emotion never reaches zero: Emotion plays some role in all human decisions.

A number of phenomena can be represented by Figure 1. It can represent the evolution of the brain, with cortical mechanisms gradually increasing in size and influence but never completely replacing subcortical systems. It can also reflect the brain's growth in fetal and later development, as ontogeny recapitulates phylogeny. The figure can also represent the development of decision-making from infancy to adulthood, with the gradual learning of language and social rules, and expectations substituting for earlier, more reflexive, controls of behavior. It can also represent a range of social relationships, from formal relationships with relative strangers on the right, and intimate relationships on the left, where spontaneous emotional communication flows easily. Figure 1 can also represent a range of situations going from the most emotional on the left to the most rational on the right.

The emotional versus rational influences of online communication media can also be represented by Figure 1. Marshall McLuhan (1964) proposed that media are technological extensions of the body that can train perceptual habits and alter patterns of thought and feeling in fundamental ways. In particular, he suggested that changes in the habits afforded by electronic media have encouraged patterns of holistic perceptual/cognitive processing as opposed to linear sequential processing. Together with the direct worldwide pooling of information, this change in perception created a new collective identity: a "global village." Buck and Powers (2011) suggested a reconceptualization of McLuhan's analysis in terms of print media affording rational symbolic communication and of electronic media affording nonvoluntary, direct, spontaneous emotional communication.

Chaudhuri and Buck (1995) followed Tucker's (1981) distinction between direct, holistic, and immediate syncretic cognition; and sequential, linear analytic cognition involving effortful information processing. They suggested that electronic media, often accompanied by emotional cues evoked by music and sound effects, encourage heuristic learning processes and may actually discourage analytic cognition. Based upon this analysis, they assessed analytic and syncretic-cognitive responses to 120

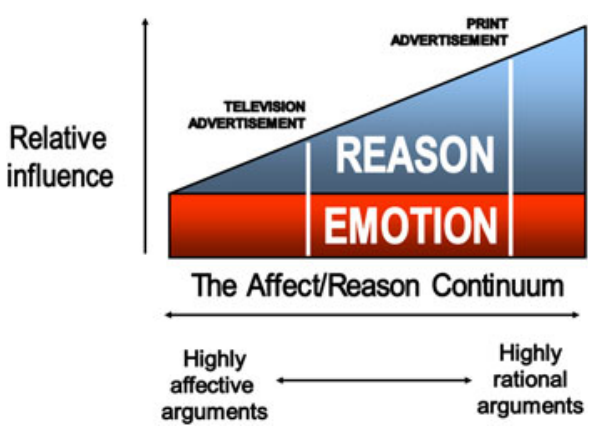

Figure 1 (Buck). The affect/reason involvement (ARI) model illustrating the interaction of reason and emotion. Behavior at the extreme left is entirely emotional and "irrational," while the relative influence of reason increases progressively toward the right. At the extreme right, reason is predominant but emotion still exerts some, often hidden influence. 
television and 120 print (magazine) advertisements. These involved 29 product categories, and a variety of characteristics were controlled, including advertising strategy and familiarity. Analytic responses (elicited from questions asking: did the ad make you think of fact, arguments, differences, etc.) were higher for advertisements in print media, while syncretic responses (elicited from questions asking: did the ad make you happy, feel good, excited, etc.) were higher for television advertisements (see Figure 1).

Adding emotion to the Bentley et al. analysis could arguably contribute to its usefulness. It is not incompatible with the cognitive explanation that this involves the relative presence or absence of rational consideration of payoffs and risks. Bentley et al. have noted that modern Western society is characterized by saturated markets in which there are thousands of extremely similar information sources and consumer products. Moreover, adding emotion to the model allows a way to consider the complex emotions - far beyond simple considerations of positive or negative valence - that can have nuanced effects on decisionmaking in a wide range of actual and consequential risky situations.

As an example, Buck et al. (2004) queried college students in America and India about emotions experienced in risky sexual situations (e.g., using or not using condoms with strangers or long-term partners; discussing condom use with a potential partner). Results indicated a wide range of significant results: for example, condom use was associated with more caring but less intimacy in both female and male respondents, using condoms made men report more feelings of anger and less power while the opposite was found for women, and patterns of response in India and America were quite similar. These results informed the design of an intervention stressing emotional education involving a filmed conversation between two persons discussing having sex, whether to use condoms, and the emotions involved. This brief intervention was found to significantly increase reported condom use six months later (Ferrer et al. 2011).

I suggest that learning about the emotions likely experienced in a risky situation ahead of time can, in effect, inoculate the individual against being "carried away" by those emotions when the risky situation presents itself. In the Bentley et al. analysis, this could constitute a way in which payoffs and risks can be made more transparent, but it goes further in suggesting specific ways in which such transparency may be achieved.

\section{Mapping collective behavior - beware of looping}

\author{
doi:10.1017/S0140525X13001696
}

\section{Markus Christen ${ }^{a, b}$ and Peter Brugger ${ }^{b}$ \\ aInstitute of Biomedical Ethics, University of Zurich, CH-8032 Zurich, Switzerland; ${ }^{\mathrm{b}}$ Department of Neurology, Neuropsychology Unit, University Hospital Zurich, $\mathrm{CH}-8091$ Zurich, Switzerland. \\ christen@ethik.uzh.ch peter.brugger@usz.ch http://www.ethik.uzh.ch/ibme/team/mitarbeitende/markuschristen.html http://www.neuroscience.ethz.ch/research/neural_basis/brugger_p}

\begin{abstract}
We discuss ambiguities of the two main dimensions of the map proposed by Bentley and colleagues that relate to the degree of selfreflection the observed agents have upon their behavior. This selfreflection is a variant of the "looping effect" which denotes that, in social research, the product of investigation influences the object of investigation. We outline how this can be understood as a dimension of "height" in the map of Bentley et al.
\end{abstract}

Maps facilitate the orientation in complex worlds, and the target article by Bentley et al. provides an excellent map to the world of human decision behavior. But maps are more than descriptive tools; they coin entities and influence the way the map makers think about the world - the information maps provide feedback to those who have been mapped. Ian Hacking established the term "looping effect" to convey the notion that when humans (as opposed to, say, molecules) are the object of investigation, they consciously react to both the process and the product of investigation.

Famously, Hacking (1992) illustrated the principle of looping by pointing out the influence of medical-psychological classification systems on the prevalence of certain health-related conditions. For instance, in North America the condition labeled "multiple personality disorder" appeared to explode in frequency after the medical community accepted it as a disease, devoted scientific conferences to the topic, and had findings and opinions regarding it disseminated among the general public. In the United Kingdom, where the same condition was regarded as an iatrogenic madness of the crowd, multiple personalities remained rare. Hacking's point was that illnesses can be transient and regional just like the classification manuals of mental diseases are bound to certain times and places. Mapping diseases is not principally different from mapping healthy human behavior, from sexual orientation to attitudes toward poverty, immigration, and violence (Hacking 1995), but also to first-name or Facebook popularity.

Given the undeniable fact that, in social research, the product of investigation thus influences the object of investigation, in what ways could looping shape the map proposed by Bentley and colleagues to describe human collective behavior? When people know that their behavior is in the southeast (using Bentley et al.'s terminology), what effect would this knowledge have? We suggest that this kind of information adds a third dimension to the map that may be captured by the analogy of height (or contour lines on geographical maps) indicating the degree of selfreflection the observed agents have upon their behavior. Even if you are in the same quadrant of the map - it is quite a different situation to be deep in a valley lacking "looping-related" insights compared to being on top of a hill indicating a high degree of self-reflection the agent (or system of agents) has with respect to their knowledge of what they know about themselves or others know about them.

We suggest that such looping-related insights indicating the degree of self-reflection refer to two types of knowledge that are related to two ambiguities inherent to the dimensions of the map proposed by Bentley et al. Their first dimension concerns the degree of social influence on the decision of the agent, with complete independence attainable at the far western side of their map and a pronounced susceptibility to mirror social expectancies at the far eastern side of their map. Going from west to east thus denotes an increase in social influence, which is associated with the ability to discern social behaviors and options associated with others' behaviors and to adopt the own behavior through mechanisms such as, for instance, imitation. The perspective of looping, however, adds an additional knowledge component to this picture, because people make models (simple theories) based on themselves as well as on other people with respect to mechanisms driving their behaviors. People may copy the behavior of others without knowing anything about why they display a particular behavior, or by having an accurate model of the mechanisms that drive their own and others' decisions. Although this modeling does not directly change observed behavior patterns, it will have an impact, as outlined below.

The second dimension in Bentley et al.'s map captures the transparency in the payoffs and risks associated with the decisions agents make. In the far north, people have full transparency on what options are available and what their associated payoffs are. In the deep south, options and their consequences are opaque. But again, we need to consider an orthogonal dimension associated with this north-south axis, one that takes looping into account. That is, it critically matters whether an agent is aware 
of whether his or her knowledge of option payoffs is accessible to third parties, too. People may have no transparency with respect to payoffs and know that others also lack this transparency - or they may not know to what extent the others know the payoffs. Again, the opacity of the payoff for each person is the same, but the two situations drastically differ.

If we quantify effects of looping as the degree of self-reflection along the two dimensions just outlined, we do not expect that the major characteristics of the behavioral pattern in terms of output distributions change (e.g., Gaussian in the northwest versus longtailed in the southeast). However, we suggest that this additional dimension helps one to understand the dynamics on this map. In a nutshell, we believe that a higher degree of self-reflection will allow for quicker movements on the map, that is, make behavioral patterns more unstable.

Having accurate knowledge (and models) of what drives others' decisions will allow for strategic decisions which-just as the "invention" of new diseases has shown - may then change the behavioral mechanisms of others, by providing novel "identities" for persons: that is, a mechanism of de-stabilization. In contrast, not knowing that others also don't know enhances the opacity of payoffs and may contribute to pluralistic ignorance. This would stabilize social dynamics, if often only in a suboptimal state. Elaborating the map analogy a bit further: A higher degree of selfreflection means standing on a mountain with a view, but risking falling down (and consequently to be relocated on the map). Finally, this analogy points to an additional aspect when taking looping into account: Increased self-reflection-also by reading sociologists' behavioral maps - may not be a positive exercise in all cases. While many situations may require an increase in self-reflection, in other situations (supported, e.g., by privacy arguments) too much self-reflection may lend a disservice to the agent (Christen et al. 2013).

\section{Modesty can be constructive: Linking theory and evidence in social science}

\author{
doi:10.1017/S0140525X13001702
}

\section{Steven N. Durlauf}

Department of Economics, University of Wisconsin at Madison, Madison, WI 53706.

sdurlauf@ssc.wisc.edu

http://www.ssc.wisc.edu/econ/Durlauf/

Abstract: This commentary argues that Bentley et al.'s mapping of shifts in collective human behavior provides a novel vision of how social science theory can inform large data set analysis.

The target article by Bentley et al., "Mapping collective behavior in the big-data era," is a fascinating paper and the authors deserve congratulations for a pioneering piece of research.

The article's important contribution is the development of a synthesis between behavioral models of the type that are standard in economics and statistical models of the type that are normally used in the analysis of large data sets. From the economic perspective, the goal of an empirical exercise is the development of an interpretive framework for observed behaviors, one in which choices and outcomes derive from well-posed decision problems. The standard "big data" analysis exploits the availability of massive data in order to develop statistical models that well characterize the data. The size of these data sets allows for a constructive form of data mining, in which the analyst allows the data to select a best-fitting model. From the perspective of an economist, the data mining exercise often appears to be a black box. Although the statistical model may have high predictive power, it does not reveal the mechanisms that determine individual choices and so is not amenable to counterfactual analysis. In contrast, from the statistician's perspective, economic models may be predicated on functional form and other assumptions that are required to operationalize a given theory, but do not have any justification outside of tractability.

Bentley et al. transcend the limitations of these approaches by showing how behavioral models may be used to understand patterns found in a range of large data sets. They achieve this by using behavioral models as an interpretive device, rather than as a literal representation of reality. In this respect, they take a more modest stance than is found in so-called structural approaches to econometrics. The authors compellingly demonstrate that this modest stance can still provide substantive social science insights. The authors consider two aspects of the determinants of decisions. The first dimension involves the respective role of individual-specific versus social factors in affecting choices; the second dimension involves the quality of information available to agents on the payoffs from actions. By partitioning environments determined by individual versus social factors and information rich versus information poor environments, one can then consider four categories of choice types. The target article shows that this "quadrant" approach allows for interpretation of differences in the properties of large data sets that are collected in disparate contexts. Bentley et al. demonstrate that these differences can be understood in terms of underlying differences in the preferences and information sets of the individuals that comprise the data.

Unlike the standard economics paper, Bentley et al.'s study does not contain any formal statistical calculations, hypothesis tests, and the like. This absence is not a reason to question the empirical contributions of the target article. Social science evidence comes in many forms. The approach taken by the authors, which uses economic theory to interpret data patterns, rather than fully explain them, is underappreciated as an integration of empirics and theory. The modesty of the theory/ empirics link respects the limits of any social science theory or set of theories as an interpretive framework for data sets of the type under study. Thus, the authors have articulated a constructive vision of "big" social science for "big" data. I look forward to their subsequent work.

\section{The crowd is self-aware}

\section{doi:10.1017/S0140525X13001714}

\section{Judith E. Fan ${ }^{\mathrm{a}}$ and Jordan W. Suchow ${ }^{\mathrm{b}}$ \\ ${ }^{a}$ Department of Psychology, Princeton University, Princeton, NJ 08544; \\ ${ }^{\mathrm{b}}$ Department of Psychology, Harvard University, Cambridge, MA 02138. \\ jefan@princeton.edu suchow@fas.harvard.edu \\ https://sites.google.com/site/judithfan/ \\ http://jordansuchow.com}

Abstract: Bentley et al.'s framework assigns phenomena of personal and collective decision-making to regions of a dual-axis map. Here, we propose that understanding the collective dynamics of decision-making requires consideration of factors that guide movement across the map. One such factor is self-awareness, which can lead a group to seek out new knowledge and re-position itself on the map.

In the target article Bentley et al. propose a framework for describing personal and collective decision-making in which decisions vary along two principal dimensions: the extent to which they are made independently versus socially, and the extent to which values attached to each choice are transparent versus opaque. They argue that in at least some domains - such as the generation and transmission of knowledge - the dynamics of how ideas are selected and propagated are approaching hive mind.

Bentley et al. use their dual-axis approach to chart an impressive range of social phenomena. Beyond assigning each phenomenon to a position on the map, however, it is important to 
examine the factors that guide movement across it (e.g., from the southeast to northeast, as social decisions gain transparency). One such factor is self-awareness: A crowd's members know the social structure and cognitive style of the group and can use this knowledge to alter the supply of information, to shift demand, and to change patterns of consumption. In doing so, the crowd guides its own trajectory.

For instance, we consider public awareness of global climate change, which lags behind a consensus among scientists that it is the result of human activities. What underlies this collective inertia? One intriguing interpretation advanced by Bentley et al. (2012) is that public discourse on the topic follows the "boomand-bust" cycles that are characteristic of the southeast: high rates of imitation and low levels of transparency. This is meant to be contrasted with the pattern of keyword usage among climate scientists, which shows a lower rate of turnover, characteristic of the northeast: strong influences of social learning, but, it is argued, more transparency about the utility of widely used terminology.

The view suggested by this comparison, featured prominently in the target article, is that the kind of group determines how ideas are transmitted between its members: scientists decide in one way, the public in another. We question the premise that collective behavior exhibited by professional knowledge-makers is fundamentally different from that of the non-specialist laity. Rather, we suggest that what distinguishes the two patterns of data-handling behavior is the degree of motivation to invest in a question and seek out or generate information to address it. Such a drive is tantamount to northward movement on the map, toward positions of reduced ignorance.

Moreover, we propose that - in this era of big data more than ever before-the crowd plots its own trajectory on Bentley et al.'s map. Such a capacity for collective mobilization is a consequence of what we term self-awareness. In this context, we take self-awareness to be a group-level analogue of metacognition in part, it is the capacity for a group to diagnose gaps in its own knowledge and to act to fill them.

How does a group gain self-awareness? As with scientific disagreement, political controversy motivates people to find gaps in each other's knowledge. This is especially true when the stakes are high. For instance, there is currently public interest in legislation that regulates labeling of genetically modified organisms (GMOs) distributed as food (Burton et al. 2001; Frewer et al. 2013). At first, public discourse on the topic may primarily rely on lay intuitions about the dangers and merits of GMOs (Mielby et al. 2012). At the same time, there is a large scientific literature that can be brought to bear on the debate (Conner et al. 2003; Kuiper et al. 2001), with interested groups taking remedial efforts by using outside knowledge to bolster their own position and identify shortcomings in those of others. These insights may broadcast widely using information technologies associated with the big-data era, broadening the scope of public understanding and sharpening the conversation among dissenting perspectives. This exchange pushes the community as a whole further north on the map.

A fruitful avenue for future research would involve tracking a single public debate longitudinally, by monitoring decisionmakers' trajectory on the map. This might capture, for example, how the dynamics of public discourse evolve from initial states of ignorance in the southeast toward later states of insight as the public becomes invested in the outcome of a particular debate.

Beyond its utility in social science research, we think Bentley et al.'s framework will be most useful insofar as it leads to greater awareness of the modes of collective decision-making. This awareness will make it possible for individuals and groups to reposition themselves on the map when faced with uncertainty. Rather than endorsing a fatalism toward the hive mind, we suggest that self-awareness is the critical factor that lends flexibility to human choices, allowing us to seek out new sources of information, adopt new modes of decision-making, and perhaps even generate new knowledge in response.

\section{Adding network structure onto the map of collective behavior}

\author{
doi:10.1017/S0140525X13001726
}

\author{
Santo Fortunato, ${ }^{a}$ Jari Saramäki, ${ }^{a}$ and Jukka-Pekka Onnela ${ }^{b}$ \\ ${ }^{\mathrm{a}}$ Department of Biomedical Engineering and Computational Science, Aalto \\ University, Fl-00076 Aalto, Finland; ${ }^{\mathrm{b}}$ Department of Biostatistics, Harvard \\ University, Harvard School of Public Health, Boston, MA 02115. \\ santo.fortunato@aalto.fi \\ onnela@hsph.harvard.edu \\ http://sites.google.com/site/santofortunato/ \\ http://becs.aalto.fi/ jsaramak/ \\ http://jponnela.com/
}

Abstract: We propose an extension to the map of Bentley et al. by incorporating an aspect of underlying network structure that is likely relevant for many modes of collective behavior. This dimension, which captures a feature of network community structure, is known both from theory and from experiments to be relevant for decision-making processes.

The target article by Bentley et al. makes an important contribution by providing a framework for categorizing different types of collective behavior. As more and more "big data" on human behavior are employed in research, these types of maps will become increasingly helpful in contextualizing old and new forms of collective behavior. The map Bentley et al. provide is based on discrete-choice approaches to decision-making, which naturally yields the east-west and north-south dimensions. Here, we make the case for an additional dimension that, based on our own work, would likely be informative in bridging microscopic behaviors and collective outcomes. This extra dimension is the network structure, or topology, on which social processes and behaviors occur.

Bentley et al. discuss the possibility of exploring the effects of other dimensions on decision-making towards the end of their article. Network structure, also mentioned by Bentley et al., has a key role in how dynamic processes unfold on social networks. For example, social influence, which by definition cannot exist in the absence of others, is particularly sensitive to the structural features of the social network in which the individuals are embedded. The effect on outcome can be dramatic. For example, Centola (2010) demonstrated empirically that the adoption rate of an online service is significantly increased for subjects whose social networks are highly clustered.

If there were an additional, (potentially) orthogonal network dimension to the north and southeast quadrants of Bentley et al.'s map, what might the extreme points of the axis represent? There are many possibilities, ranging from networks with narrow to broad connectivity distributions and from highly clustered to unclustered networks. However, for decision-making processes where social influence plays a role (Onnela \& Reed-Tsochas 2010), we argue that the key feature is social group structure that limits the number of options available for social learning. We propose a network dimension (see our Figure 1), where in one extreme the network has multiple cohesive, fully connected social groups, but the groups themselves are linked by only a very small number of ties. In this case, social influence mainly originates from within each group. In the other extreme, we have fully connected networks where everyone is connected to everyone else, either directly or indirectly, such that each individual is able to observe everyone else's choices (e.g., Wikipedia). In between, we have networks that retain some group structure, but where highly connected hub nodes are able to influence members in several groups (e.g., Twitter). 


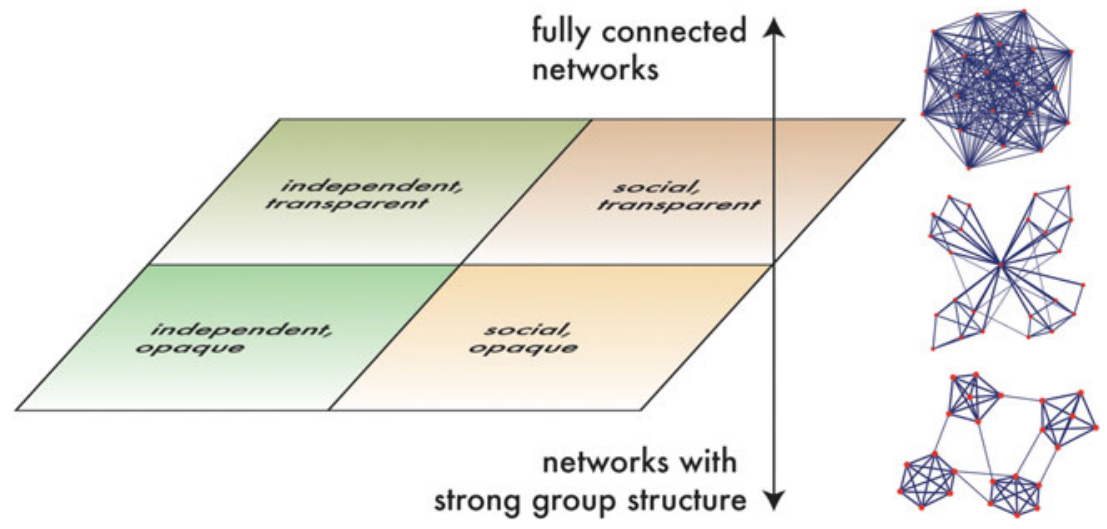

Figure 1 (Fortunato et al.). A proposed network dimension, relevant to the northeast and southeast quadrants. At one extreme are networks with very strong social group structure, limiting access to choices for social learning, and at the other extreme are networks where everyone is connected to everyone else.

As an example, consider the part of this augmented map with strong social group structure and purely social learning ("I'll have what she is having") where each individual imitates a random network neighbor. This is the same mechanism as in the voter model of statistical physics (Castellano et al. 2009). In this example, depending on the details of the decision-making process, the collective outcome may or may not depend on network structure. If the individuals update their choices perpetually as above, the model leads to a consensus where, regardless of network structure, a single choice prevails. However, if the individuals choose only once and stick to their choice, the distribution of the final popularity of choices heavily depends on network structure (Fortunato \& Castellano 2007). Even with repeated choice updating, if an undecided state is added to the model, strong social group structure gives rise to long-lasting metastable states with each group sticking to its own choice (Toivonen et al. 2009). If each individual were to always pick the majority choice in its network neighborhood, each group would converge to its own choice, largely unaffected by those of other groups.

Consider now the other extreme of the network dimension, that is, a fully connected network where each individual sees the choices of all others. Here, blind imitation by copying the choice of a randomly chosen individual is equivalent to picking a decision with probability proportional to its popularity in the population. Thus, the mechanisms attributed to southeast (blind imitation) and northeast (choosing on the basis of popularity) are the same. This is preferential attachment, yielding a lognormal popularity distribution if the adoption probabilities are subject to random fluctuations, as Bentley et al. state. However, in the absence of such fluctuations, the resulting popularity distribution is a power law (Barabási \& Albert 1999). Indeed, the profile of the popularity distribution of Wikipedia pages is consistent with a power law, not with a lognormal distribution (Ratkiewicz et al. 2010).

We would also like to argue that the distinction between the northeast and southeast quadrants (transparent vs. opaque payoffs) may at times be difficult as the payoffs may be socially generated-unrelated to intrinsic qualities of the options, but instead a product of how social influence is mediated through the network. Watts (2011) points out how the success of hits in different sectors of human activity, such as art (Mona Lisa), literature (the Harry Potter series), and technology (the iPod) caught experts by surprise. For example, eight publishers rejected the first Harry Potter manuscript. Experiments by Salganik and colleagues (Salganik et al. 2006) showed that the same set of songs were ranked differently by comparable groups of subjects depending on the extent to which they were exposed to the decisions of others. The difference between a smash hit and failure may then be due to social cascades arising from initial random fluctuations, giving the incipient winner a decisive early advantage over its competitors (Fig. 1).

\section{Missing emotions: The Z-axis of collective behavior}

\author{
doi:10.1017/S0140525X13001738
}

\section{Alejandro N. García, José M. Torralba, and Ana Marta González}

Institute for Culture and Society, Edificio de Bibliotecas, Universidad de Navarra, 31080 Pamplona, Spain.

angarcia@unav.es jmtorralba@unav.es agonzalez@unav.es http://www.unav.es/centro/cemid

Abstract: Bentley et al. bypass the relevance of emotions in decisionmaking, resulting in a possible over-simplification of behavioral types. We propose integrating emotions, both in the north-south axis (in relation to cognition) as well as in the west-east axis (in relation to social influence), by suggesting a $\mathrm{Z}$-axis, in charge of registering emotional depth and involvement.

Emotions influence both individual and collective behavior. Yet, in their account of collective behavior, Bentley et al. do not mention emotions even once, and it is not clear how they could be integrated into their proposal such that it would truly account for the discrete decisions that individuals face. The two axes they propose for organizing the analysis of big data are meant to measure the degree of social influence and transparency of payoffs in discrete choices, yet the way individual choices are influenced by emotions cannot simply be assimilated by any of the given variables. It has become increasingly clear that economic decisions cannot be explained without taking the emotions into account (Berezin 2005; 2009). While Bentley et al. recognize that "the map requires a few simplifying assumptions to prevent it from morphing into something so large that it loses its usefulness" (target article, sect. 2, para. 7), we argue that neglecting emotions can only result in a distorted and impoverished account of behavioral types, which reduces, if not spoils, the usefulness of the map altogether. We need "more sensitive methodologies with which to capture these complex multidimensional decision-making processes" (Williams 2000, p. 58).

Perhaps Bentley et al. have ignored emotions on the basis of the assumption that they fall into the category of "opaque and socially influenced behavior." But this assumption would be wrong, for emotions do not only influence cognition and decision making; they are also present, in varying degrees, in seemingly independent behavior. By not explicitly including the role of emotions in decision making, Bentley et al. are likely to have arrived at conclusions based on spurious variables.

The way emotions influence cognition cannot be adequately represented in terms of transparency versus opacity of the payoffs and risks along the north-south axis. While there are cases of "collective effervescence" (Durkheim 2001, p. 171) in which 
emotions influence cognition to the point that they obscure any thought of the consequences of a given action, at other times emotion does not obscure the recognition of consequences and yet agents still decide to act regardless of the consequences. Think, for example, of someone who sacrifices something for a friend: While it might be said that in this case the agent weighs two options and finds one of them more rewarding than the other, there is room to argue that emotions cannot be accounted for in terms of a cost-benefit analysis (Archer \& Tritter 2000). To give a more plausible account of behavioral types that indirectly reflects personal and cultural values (Hechtman et al. 2012), emotions should be considered as an independent variable. Thus, although a particular agent might have clear knowledge of the objective costs and benefits involved in a particular choice, her decision may not be determined by those considerations. Indeed, it is completely plausible that the emotional variable sometimes trumps other variables in the decision-making process.

Yet, in addition to their role as independent variables, emotions can influence the cognitive process itself, that is, cognitive evaluation is not independent of emotional dimensions. The information we consider relevant in a given context depends on emotional states. As Bandelj (2009) notes:

[E]motions serve as one of the chief mechanisms to constrain and direct our attention, and hence frame our decisions. Emotions define what shall be considered as relevant for any particular action problem. In addition, during the process of selecting optimal means for desired goals, emotions help us narrow down the range of plausible alternatives and help us rank these alternatives. (p. 352)

The level of emotional involvement regulates cognitive processes as well as what counts as a cost or a benefit in a particular situation. Accordingly, transparency and opacity do not depend only on the objective information provided, but also on the degree of emotional involvement. Cognitive transparency is not equivalent to "emotional detachment"; certainly, very often it is only through emotions and the evaluations they entail, that is, through some degree of emotional involvement, that we come to realize the seriousness of certain injustices.

Presence of emotions along the west-east axis cannot be reduced to the "opaque and socially influenced behavior." It is certainly true that emotions are present in socially influenced behavior - if only to avoid cases of "cognitive dissonance" (Festinger 1964, p. 5). From this perspective, we could even inquire into the extent to which the Internet influences emotional reactions to events and, hence, the very nature of big data collected through it. However, reducing the presence of emotions to the quadrant of "opaque and socially influenced behavior" would be misleading. This is so for two reasons: first, because emotions can also be the motive for isolationist behavior, which at first sight could resemble independent behavior, and second, because highly independent and calculated decisions are sometimes made precisely to create some sort of emotional bond, and it is precisely the Internet, with its extensive social media, which often serves this purpose (Illouz 2007).

In light of these considerations, we think that Bentley et al.'s empirical framework for big-data research would benefit from introducing a $Z$-axis that registers the intensity of emotions influencing individual choices at any given moment. While this inclusion entails complicating the behavioral types (see graph in our Fig. 1), the result not only provides a more plausible account of human behavior, but arguably better serves the practical ends that the authors advance at the end of the article. After all, as marketing researchers know well (Bagozzi et al. 1999), when decisions are mostly based on emotions, providing too much information may be counterproductive; the important

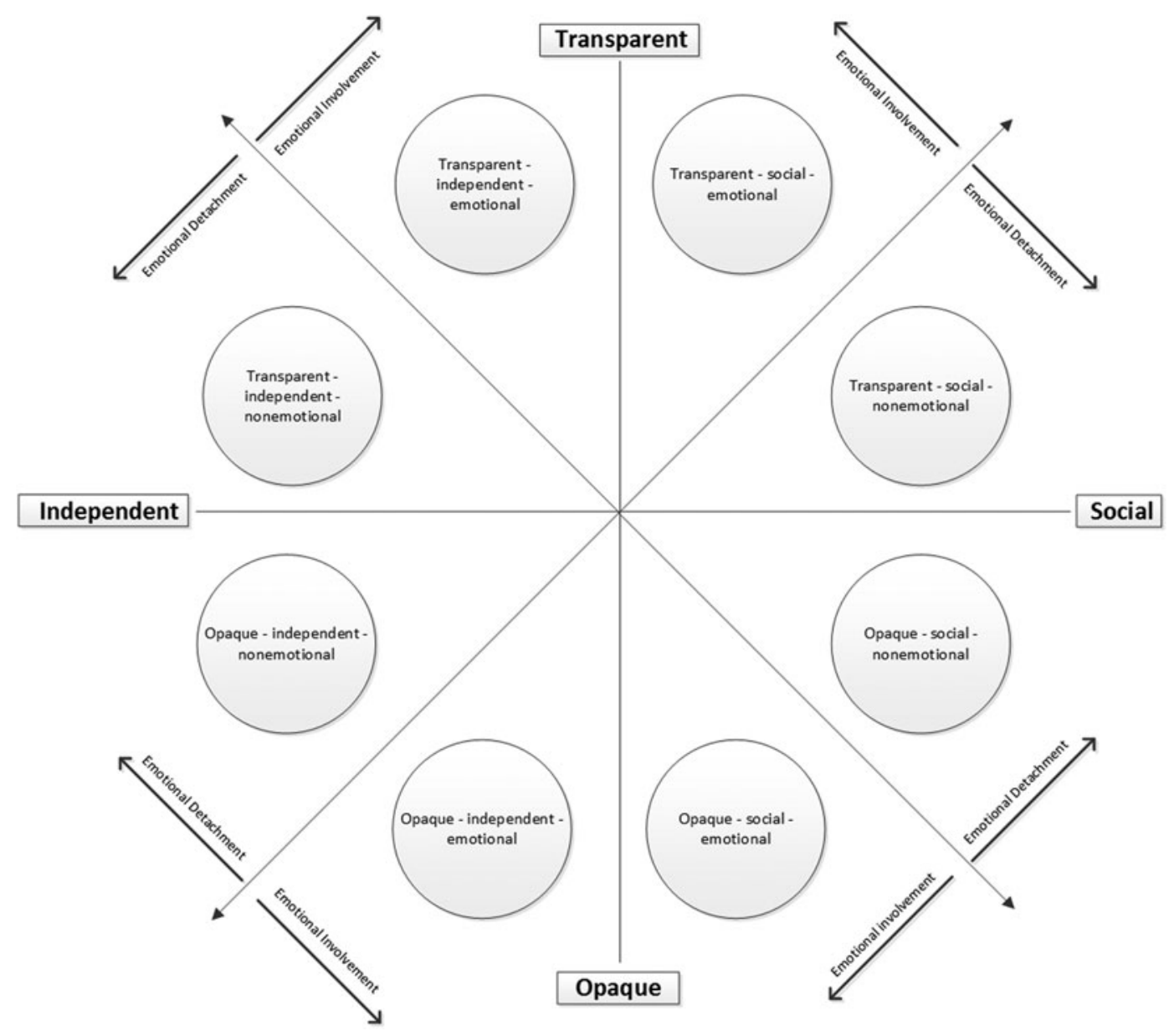

Figure 1 (Garcia et al.). Graph 1: A tentative reformulation of behavioral types that includes emotions. 
thing, then, is not to provide too much information, but rather to provide the information relevant to the agent (see Fig. 1).

\section{Capturing the essence of decision making should not be oversimplified}

\author{
doi:10.1017/S0140525X1300174X
}

\section{Ewa Joanna Godzińska and Andrzej Wróbel PL 02-093 Warsaw, Poland. \\ e.godzinska@nencki.gov.pl a.wrobel@nencki.gov.pl \\ http://www.nencki.gov.pl/en/laboratory-of-ethology \\ http://vslab.nencki.gov.pl}

Department of Neurophysiology, Nencki Institute of Experimental Biology PAS,

\begin{abstract}
Bentley et al. propose a thought-provoking approach to the question of causal factors underlying human choice behavior. Their map model is interesting, but too simplified to capture the essence of decision making. They disregard, among other matters, qualitative differences between various subcategories of social influences, and the role of neurobiological factors engaged in interdependent individual and social decision-making processes.
\end{abstract}

Bentley et al. have created a simple map-like model of selected determinants of human decision-making behavior, focusing attention on two factors: (1) the degree to which a decision is independent versus socially influenced (axis 1 of the map), and (2) the degree of transparency concerning the consequences of various decisions in terms of payoff and/or risk (axis 2). We agree that these factors play an important role in human decisionmaking processes, but we are not convinced that the map model of Bentley et al. will allow us, as stated by its authors, to "capture the essence of decision making" (target article, Abstract). Below we discuss the main shortcomings of their model.

Many aspects of the map model of Bentley et al. are simplified, some of them deliberately. The authors argue that "the map requires a few simplifying assumptions to prevent it from morphing into something so large that it loses its usefulness for generating potentially fruitful research hypotheses" (target article, sect. 2, para. 7). However, such an approach raises a question as to what degree such a simplified model still provides a useful representation of the analyzed phenomena. The authors seem to share these doubts, as they recommend further research to explore the effects of factors deliberately disregarded in their model.

Extensive research in the field of behavioral biology and social psychology has yielded numerous data demonstrating that social influences mediating choice behavior of animals and humans show a high degree of qualitative diversity. Bentley et al. are aware of the existence of various subcategories of social influences and name some of them (copying, verbal instruction, imitation). However, they quantify the impact of social influences on decision making in a very simplified way, solely by providing information on the degree to which a decision is socially influenced. Qualitative differences between various subcategories of social influences are disregarded. As a consequence, a decision half-influenced by imitation of decisions of other agents ("herdlike" behavior) and a decision influenced to the same degree by avoidance of imitation (nonconformist behavior) would occupy the same position on their map. Distinctions between different strategies employed in decision making are thus blurred instead of being emphasized. We may also note that the authors tend to identify social linking with herdlike behavior and pay little or no attention to social interactions leading to enhanced diversity of behavior.

Yet another set of problems is related to the fact that the map model proposed in the target article represents a continuous space defined by the two analytical dimensions, but the authors have divided it into four quadrants "for ease of discussion and application to example datasets" (target article, sect. 2, para. 7), and throughout their article they discuss various social phenomena mostly by assigning them to one of these quadrants, without any attempt to identify their exact position on the map. So, ultimately, they mostly use a discrete $2 \times 2$ matrix and not a continuous map. Such simplification has, however, some merits, too, as it puts aside the need to quantify precisely the analyzed phenomena by assigning to them the values of variables representing the two axes of the map, and it is far from obvious how to make the measurements of values of these variables or even to provide reliable estimations of them. However, that question must find a satisfactory solution if Bentley et al.'s map model is to be assigned to the domain of empirical science and give rise to testable hypotheses.

The authors also pay no attention to the fact that the degree of transparency concerning the consequences of various decisions is socially influenced. Therefore, the two axes of the map model of Bentley et al. are not independent: social influences may affect the choice process in two ways, directly (axis 1) and indirectly (axis 2). This raises an additional difficulty in assigning values to variables forming the axes of the map model. We should also bear in mind that information obtained from other individuals may enhance the transparency concerning the consequences of decisions, but it may also be involuntarily or purposefully misleading (the phenomenon of cheating).

Finally, the map model of causation of decision-making processes takes into account only causal factors related to the individual and/or social level of organization. Phenomena and processes taking place on lower levels of organization are disregarded. However, factors selected by Bentley et al. to explain the causation of human decision making processes exert their influence via modifications of neurobiological processes taking place in the agent's brain. Our knowledge about the neurobiological basis of animal and human choice behavior is already quite advanced. We are convinced that the processes of decision making could be much more profoundly understood if such issues as the contextual modulation of behavioral choice (including, in particular, the relative contribution of conscious processes vs. processes induced by subliminal stimuli) (Block 2007; Palmer \& Kristan 2011), the role of particular brain structures and neurotransmitter/neuromodulatory systems (Forbes \& Grafman 2013; Jung et al. 2013; Pleger \& Villringer 2013; Yu \& Dayan 2005), and the role of synchronization of electrical activity rhythms in various parts of the brain (Guitart-Masip et al. 2013) were taken into account. In our opinion, Bentley et al.'s approach would be more successful if it were supplemented by information showing how the impact of factors representing analytical dimensions of their map is translated into neurobiological processes underlying decision making. Further refinements providing additional information on neurobiological correlates of human choice processes might make their map model still more useful and realistic.

\section{Conflicting goals and their impact on games where payoffs are more or less ambiguous}

\section{doi:10.1017/S0140525X13001751}

Astrid Hopfensitz, ${ }^{\mathrm{a}}$ Emiliano Lorini, ${ }^{\mathrm{b}}$ and Frederic Moisan ${ }^{\mathrm{a}, \mathrm{b}}$
${ }^{\mathrm{a}}$ Toulouse School of Economics, 31000 Toulouse, France; ${ }^{\mathrm{b}}$ Institut de
Recherche en Informatique de Toulouse (IRIT), Toulouse University, 31062
Toulouse, France.
Astrid.Hopfensitz@tse-fr.eu http://idei.fr/member.php?i=1706
Emiliano.Lorini@irit.fr http://www.irit.fr/ Emiliano.Lorini
fmoisan@gmail.com http://irit.academia.edu/FredericMoisan

Abstract: The two-dimensional map by Bentley et al. concerns decisionmaking and not games. The east-west dimension is interpreted as the level at which individuals identify with some larger group. We think that this should be linked to the concept of social ties. We argue that social ties will lead to different outcomes in the "north" compared to the "south."

Bentley et al. present a two-dimensional map allowing a categorization of decisions dependent on the amount (and precision) of 
Commentary/Bentley et al.: Mapping collective behavior

information about payoffs and the degree to which some larger group is influencing agents. Among other applications, they discuss the applicability of this model to friendship networks and language use. However, both situations imply some kind of interaction with others. We therefore think that the model has to be extended and discussed for decisions made in games. In games, final outcomes are determined by the decisions taken by more than one player.

While the north-south dimension is easily extended to games where payoffs are either known or ambiguous, we have to be specific as to what the east-west dimension would mean. Bentley et al. consider the east as the "social" quadrants where $J_{t}$ is high, with $J_{t}$ denoting a "social-influence parameter that the fraction of people [...] in an individual's peer group [...] has on the person" (target article, sect. 2, para. 2). If we are talking about games, these social links will influence behavior through two different channels. On the one hand, decisions might be influenced by the behavior that is observed by others. On the other hand, decisions will be influenced by the specific social link agents have with their interaction partner. While the first point is more or less equivalent to the impact discussed by the authors for individual decisions, we think that the second part needs to be included in the model.

If an agent is interacting with someone from his peer group where $J_{t}$ is high, his own behavior will be influenced by how much individuals care about the outcomes to the group and by considerations of "what everybody should do for the best of the group." We call this the impact of "social ties" on behavior. This impact can be modelled as follows in the case of a two-agent interaction. Let us consider a strategic game with two agents $i$ and $j$ in which, as usual, $S_{i}$ and $S_{j}$ respectively denote agent $i$ 's set of strategies and agent $j$ 's set of strategies, and $U_{i}$ and $U_{j}$ respectively denote agent $i$ 's utility function and agent $j$ s utility function over the set of strategy profiles $S_{i} \times S_{j}$. Moreover, let us assume that the degree of the social tie between agent $i$ and agent $j$ is a numerical value $k_{i j}$ in the interval $[0,1]$. The following equation models how much the existing social tie between agent $i$ and agent $j$ influences the utility of a certain strategy profile $\left\langle s_{i}, s_{j}\right\rangle$ for $i, U_{i}^{S T}\left(s_{i}, s_{j}\right)$ being the transformed utility of the strategy profile $\left\langle s_{i}, s_{j}\right\rangle$ for agent $i$ which integrates the influence of the social tie between $i$ and $j$ on $i$ 's current motivations:

$$
U_{i}^{S T}\left(s_{i}, s_{j}\right)=\left(1-k_{i j}\right) U_{i}\left(s_{i}, s_{j}\right)+k_{i j} \max _{s_{j}^{\prime} \in S_{j}} U_{\langle i, j\}}\left(s_{i}, s_{j}^{\prime}\right)
$$

The idea of our model of social tie is that, in the presence of a social tie between two individuals $i$ and $j$, agent $i$ will be motivated to maximize the benefit of the group, represented by collective utility $U_{\{i, j\}}\left(s_{\mathrm{i}}, s_{j}^{\prime}\right)$, assuming that agent $j$ is also motivated to maximize the benefit of the group $\{i, j\}$. In particular, when the strength of the social tie between agent $i$ and agent $j$ is maximal (i.e., $k_{\mathrm{ij}}=1$ ), $i$ and $j$ do not face a strategic problem anymore. Indeed, the utility of the strategy profile $\left\langle s_{i}, s_{j}\right\rangle$ for agent $i$ becomes independent of player $j$ 's part in this strategy profile (i.e., $s_{j}$ ). The model is agnostic as to how the collective utility function $U_{\{i, j\}}$ should be computed, as it might be defined either in terms of a utilitarian notion of global efficiency, for example:

$$
U_{\{i, j\}}\left(s_{i}, s_{j}^{\prime}\right)=U_{i}\left(s_{i}, s_{j}^{\prime}\right)+U_{j}\left(s_{i}, s_{j}^{\prime}\right)
$$

or in terms of a Rawlsian criterion of fairness, for example:

$$
U_{\{i, j\}}\left(s_{i}, s_{j}^{\prime}\right)=\min \left\{U_{i}\left(s_{i}, s_{j}^{\prime}\right), U_{j}\left(s_{i}, s_{j}^{\prime}\right)\right\}
$$

or in terms of equality, for example:

$$
U_{\{i, j\}}\left(s_{i}, s_{j}^{\prime}\right)=\frac{1}{\mid U_{i}\left(s_{i}, s_{j}^{\prime}\right)-U_{j}\left(s_{i}, s_{j}^{\prime}\right)} .
$$

To illustrate the importance of different strengths of social ties on behavior, we studied the following asymmetric coordination game with outside option (see our Figure 1). In this sequential game, A moves first and chooses between an outside option or entering a second stage that consists of playing a coordination game with

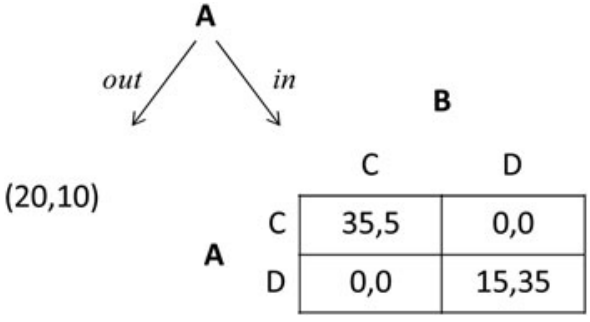

Figure 1 (Hopfensitz et al.). Coordination game with outside option and known payoffs.

B. Entering the game (choosing IN for player A) is only interesting if he or she believes that coordination will be achieved in the second stage of the game. One way to achieve coordination is by a "forward induction" argument that implies that since A chose IN, player B knows that player A will choose option C. However, coordination can also be achieved if both players agree that the $\mathrm{D} / \mathrm{D}$ outcome is the best for the group and if they both know that they care about the group.

To test whether players play this game differently when they are more or less tied to their opponent, we proposed this game to participants that were either interacting with a member of a sports team of which they were also members (team) or with another student from their university (university) (Attanasi et al. 2013). While in both cases partners belong to the same group, participants from the same team are much stronger socially tied than students from the same university. And, indeed, we see that a significantly larger proportion of participants decide to enter the game when they interact with a fellow team member and that a larger proportion coordinates in this case on playing $\mathrm{D}$ (see Table 1).

Now imagine the same game played for the southern part of the map where payoffs are ambiguous. This can be achieved by not having precise payoffs but some general information about the characteristics of each outcome, as Figure 2 shows. By obscuring the precise payoffs, coordination might now become easier for socially tied individuals since it is clearer what the optimal outcome is for the group. We would therefore expect that in such games coordination and thus efficiency will be increased

Table 1 (Hopfensitz et al.). Experimental results for interactions by sport-team members and students from the same university

\begin{tabular}{llll}
\hline \hline (70 observations) & Team & University & $\begin{array}{l}\text { Wilcoxon signed } \\
\text { rank test }(p \text { values })\end{array}$ \\
\hline A choosing IN & $62 \%$ & $42 \%$ & 0.004 \\
A choosing D & $65 \%$ & $53 \%$ & 0.033 \\
B choosing D & $77 \%$ & $64 \%$ & 0.050 \\
\hline \hline
\end{tabular}

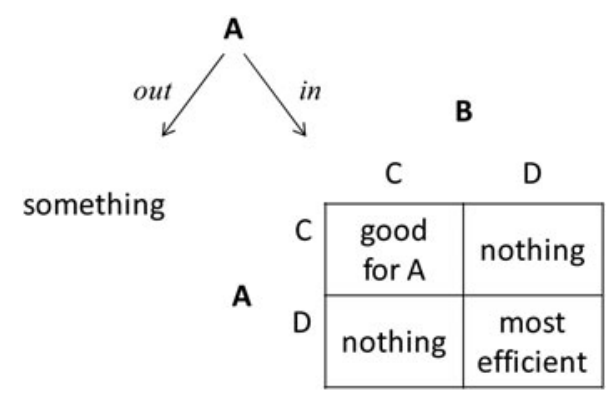

Figure 2 (Hopfensitz et al.). Coordination game with outside option and ambiguous payoffs. 
when we move to the south of the map. Thus, while Bentley et al. consider the south-east of their map as a region of "herding" that will not reach any efficient outcome if everybody is just following the others, we think that the south-east, if interpreted as a region with very ambiguous payoffs but where players are strongly tied, can lead to more efficiency than the north.

\section{ACKNOWLEDGMENT}

Funding through the Agence Nationale de la Recherche (ANR: 010 JCJC 180301 ) is gratefully acknowledged.

\section{It's distributions all the way down!: Second order changes in statistical distributions also occur}

doi:10.1017/S0140525X13001763

\author{
Mark T. Keane ${ }^{a}$ and Aaron Gerow ${ }^{b}$ \\ ${ }^{a}$ School of Computer Science and Informatics, University College Dublin, \\ Belfield, Dublin 4, Ireland; 'b School of Computer Science and Statistics, \\ University of Dublin, Trinity College, College Green, Dublin 2, Ireland. \\ mark.keane@ucd.ie gerowa@tcd.ie \\ www.csi.ucd.ie/users/mark-keane \\ www.scss.tcd.ie/ gerowa
}

\begin{abstract}
The textual, big-data literature misses Bentley et al.'s message on distributions; it largely examines the first-order effects of how a single, signature distribution can predict population behaviour, neglecting second-order effects involving distributional shifts, either between signature distributions or within a given signature distribution. Indeed, Bentley et al. themselves under-emphasise the potential richness of the latter, within-distribution effects.
\end{abstract}

It has been reported, possibly as apocrypha, that when South Sea islanders were asked to explain their cosmology about what supported the World Turtle (who supported the world), one bright spark replied: "It's turtles all the way down!" The take-home message from the target article is analogously: "It's distributions all the way down!" Though the message may seem obvious, it is interesting to note that most of the current textual, big-data literature either doesn't get it or fails to appreciate its richness.

The lion's share of the textual, big-data literature (that analyses, e.g., words, sentiment terms, tweet tags, phrases, articles, blogs) really concentrates on what could be called first-order effects, rather than on second-order effects. First-order effects are changes in a single, statistical distribution of some measured text-unit (word, article, sentiment term, tag) - usually from some large, unstructured, online dataset - that can act as a predictive proxy for some population-level behaviour. So, for instance, Michel et al. (2011) show how relative word frequencies in Google Books can reflect cultural trends; others have shown that distributions of Google search-terms can predict the spread of flu (Ginsberg et al. 2009) and motor sales (Choi \& Varian 2012), and the numbers of news articles and of tweet rates can both be used to predict movie box-office revenues (Asur \& Huberman 2010). In these cases, the actual distribution of the text data is directly reflected in some population behaviour or decision.

Second-order distributional effects are changes across several, separate distributions that can predict population-level behaviour; usually, where these separate distributions span different time periods. Big-data research on such effects is a lot less common; to put it succinctly, most of the work focuses on either the wisdom of crowds (Surowiecki 2005) or herd-like behaviour (Huang \& Chen 2006) but not on how a wise crowd becomes a stupid herd (for rare exceptions, see Bentley \& Ormerod 2010; Bentley et al. 2012; Onnela \& Reed-Tsochas 2010; Salganik et al. 2006). Bentley et al. recognise the importance of such second-order effects in stressing that population decision- making may shift from one signature distribution to another. However, we believe that there is more to be said about such second-order effects.

For instance, second-order effects can be further divided into between-distribution and within-distribution effects. Between-distribution effects concern the types of changes, mentioned by Bentley et al., where there is change from one signature distribution to another over time (movement between the quadrants of their map): for example, from the Gaussian distribution of the wisdom of crowds to the power-law distribution of herd behaviour. In physical phenomena, such distributional changes are often cast as phase transitions; for example, when water turns to ice or when a heated iron bar becomes magnetised (see Barabasi \& Gargos 2002). Bentley et al. raise the prospect that there are a host of similar phase transitions in human population behaviour, transitions that can be tracked and predicted by mining various kinds of big data. We find this prospect very exciting, given the notable gap in the big-data literature on such effects.

Bentley et al. say a lot less about within-distribution effects, the systematic changes that may occur in the properties of a given signature distribution from one time-period to the next; for example, the specific properties of separate power-law distributions of some measured item might change systematically over time. Research on such within-distribution effects in textual, big-data research is even rarer than that on between-distribution effects - one of these rarities being our own work on stock-market trends (Gerow \& Keane 2011). In this study (Gerow \& Keane 2011), we found systematic changes in the power-law distributions of the language in finance articles (from the BBC, the New York Times, and the Financial Times) during the emergence of the 2007 stock-market bubble and crash, based on a corpus analysis of 18,000 online news articles over a 4 -year period (10M+ words). Specifically, we found that week-to-week changes in the power-law distributions of verb-phrases correlated strongly with market movements of the major indices; for example, the Dow Jones Index correlated $(r=0.79)$ with changes in the 8 -week-windowed, geometric mean of the alpha terms of the power laws. These within-distribution changes showed that, week-on-week, journalists were using a progressively narrower set of words to describe the market, reporting on the same small set of companies using the same overwhelmingly positive language. So, as the agreement between journalists increases, the power-law distributions change systematically in ways that track population decisions to buy stocks.

In Bentley et al.'s map, this analysis is about tracking movement within the southeast quadrant. Naturally, we also find the prospect of such within-distribution effects very exciting, especially given the lack of attention to them thus far.

So, it really is "Distributions all the way down!" Current research is only scraping the surface of the possible distributional effects that may be mined from various online sources. Bentley et al. have provided a framework for thinking about such second-order effects, especially of the between-distribution kind, but there is also a whole slew of within-distribution phenomena yet to be explored.

\section{Keeping conceptual boundaries distinct between decision making and learning is necessary to understand social influence}

\section{doi:10.1017/S0140525X13001775}

\section{Gaël Le Mens}

Department of Economics and Business, Universitat Pompeu Fabra, 08005 Barcelona, Spain.

gael.le-mens@upf.edu

http://www.econ.upf.edu/ glemens/

Abstract: Bentley et al. make the deliberate choice to blur the distinction between learning and decision making. This obscures the social influence 
mechanisms that operate in the various empirical settings that their map aims to categorize. Useful policy prescriptions, however, require an accurate understanding of the social influence mechanisms that underlie the dynamics of popularity.

The two-dimensional "map" proposed by Bentley et al. relies on a set of four simplifying assumptions - three of which I find unproblematic. But I would like to discuss the third assumption: the simplification that consists in blurring "the distinction between learning and decision making” (target article, sect. 2, para. 7). Although the authors recognize that these are distinct actions, they also claim this distinction is somewhat too subtle to be of serious consideration in the target article. This simplification unfortunately (1) obscures the social influence processes that operate in the various empirical settings the map aims to categorize, thus limiting the usefulness of the potential policy implications of the studies being mapped; and (2) it limits our potential understanding of the implications of the new technologies underlying the "big data" revolution for the dynamics of popularities of choices and opinions.

Distinction between learning and decision making and social influence mechanisms. To understand why it is crucial to keep a proper distinction between learning and decision making, let us focus on the southeast part of the map. This quadrant concerns settings characterized by a high level of social influence and a low level of clarity of the values of the alternatives. Such settings are characterized by a high level of unpredictability (Salganik et al. 2006) and highly skewed popularity distributions. Why would an alternative become much more popular than other available choice alternatives? The target article explains this by invoking a high social-influence parameter $\left(J_{t}\right)$. But what the article does not fully acknowledge is that the dynamics of the system will strongly depend on how learning (i.e., the dynamic updating of quality estimates by the agents) and decision making (i.e., the choice between alternatives) combine in driving social influence.

Suppose, for example, that agents are motivated to select the same alternative as others (due to network externalities, for example), and coordinating a switch to a different, but potentially superior alternative is difficult (maybe because there are communication issues, or high switching costs). In this situation, learning does not matter, because the driving social influence mechanism is a coordination failure. Consequently, there will be a low turnover among the most popular alternatives, independently of the transparency of the qualities of the alternatives (Arthur 1989).

Contrast this situation to what happens in settings where the main social influence mechanism is social learning. In such settings, agents make inferences about the relative qualities of the alternatives on the basis of relative popularities because they do not have enough information to know which alternative is the best. They thus select what is popular. Prior research has shown that convergence toward one potentially suboptimal alternative relies on a very small amount of information. This implies that the release of a small amount of contradictory public information can shatter the "cascade" (Bikhchandani et al. 1992). More generally, when the dominant social influence mechanism is social learning, this suggests that there will likely be large swings in popularities and thus a high turnover rate among popular alternatives.

The prediction regarding turnover again differs if social influence operates through sampling (Denrell \& Le Mens 2007; 2013). Such social influence operates when agents are uncertain about the qualities of the alternatives: They are motivated to select what is popular (maybe because there are network externalities), but they learn about the qualities of the alternatives from their own experiences only, maybe because they do not trust others, or agents have different skills or tastes. In such settings, choices and beliefs will tend to coalesce around one alternative. But because beliefs will tend to justify differences in popularities as a result of an information bias in favor of popular alternatives, what has become popular will tend to remain popular. There will thus be a low turnover among the most popular alternatives.
What can be done by a policy maker who wants to affect the dynamics of popularities depends on the precise nature of the social influence process, which, in turn, depends on the articulation of beliefs and choices. Suppose our policy maker intends to increase the popularity of an alternative that is currently unpopular, but of higher quality than the most popular alternative. If the population is subject to a lock-in due to a coordination failure, the solution is to create a coordination mechanism (possibly a new institution, or a vote) to facilitate the switch. If a suboptimal alternative is the most popular as a result of an information cascade, making available a small amount of information in favor of the unpopular but superior alternative should be enough to lead to a switch. Such information release would not lead to a switch if the problem were a coordination failure. And if the population selects a suboptimal alternative as a result of social influence through sampling, the remedy is to make people sample the unpopular alternative. This will work even if persuasive campaigns are ineffective.

Decisions about information feeds and the technologies at the core of the "big data" revolution. The technologies that support the "big data" discussed by Bentley et al. do not only facilitate access to more information more quickly, but they are also characterized by a high level of customizability: Agents are now free to configure information sources in order to get selectively exposed to the information they want to be exposed to. They can do so by tweaking the settings of RSS (Rich Site Summary) readers, selecting the tweets they attend to, or customizing the news feed on their Facebook account. This is in sharp contrast with older forms of information broadcasting such as television, radio, or newspapers where agents were receivers with less latitude in deciding what to get exposed to. The novel customizability of information sources has the potential to reduce rather than increase the diversity of information agents get exposed to. We researchers can only hope to understand the implications of this increased customization for the dynamics of opinions, tastes, and popularity if we decouple learning, beliefs, and decision making in our analyses and aim to understand the dynamic process by which agents adjust their exposure to information.

\section{Alternative maps of the world of collective behaviors}

\section{doi:10.1017/S0140525X13001787}

\section{Robert J. MacCoun}

Goldman School of Public Policy, University of California at Berkeley, Berkeley, CA 94720-7320.

maccoun@berkeley.edu

http://conium.org/ maccoun/

Abstract: I compare the collective behavior map proposed by Bentley et al. ("BOB" for short) with a similar "balance of pressures" (BOP) map proposed by MacCoun (2012). The BOB and BOP maps have important points of convergence, but also some differences. The comparison suggests that they are analogous to different map "projections" for maps of Earth - different ways of simplifying a complex reality.

The Age of Big Data holds the promise of great discoveries, but Bentley et al. make a strong case that we'll need a good map if we want to avoid aimless wandering, and they outline an impressive candidate: their map of collective behavior (henceforth "BOB"). Recently, in Psychological Review, I offered a similar "map" of social influence based on a family of logistic threshold models called BOP ("Balance of Pressures" or "Burden of Proof"; MacCoun 2012; also see Kerr \& MacCoun 2012; MacCoun et al. 2008). In this brief commentary, I compare and contrast the $\mathrm{BOB}$ and $\mathrm{BOP}$ maps, highlighting important points of convergence and possible divergence. 
Until the early 20th century, alternative world maps conflicted, often profoundly. But the comparison of the BOB and BOP maps may be more analogous to the creative tension between alternative "projections" for transforming the 3D surface onto a $2 \mathrm{D}$ plane. Snyder (1993, p. 1) notes that "literally an infinite number of map projections are possible ... The designer of a map projection tries to minimize or eliminate some of the distortion, at the expense of more distortion of another type, preferably in a region of or off the map where distortion is less important."

The BOP map. BOP (MacCoun 2012) is a family of logistic threshold models sharing a common set of parameters. For example, the $b B O P$ model is:

$$
p_{\Delta}=\frac{1}{1+e^{-c\left[\frac{S}{N}-b\right]}}
$$

where $p_{\Delta}$ is the probability of changing one's position or practice, $S / N$ is the proportion of the population that holds the opposite position (the "sources"), $b$ is a threshold parameter, and $c$ is a "norm clarity" parameter, inversely related to the standard deviation of the threshold. In some contexts, the numerator can be replaced by $m$, a ceiling parameter, with some loss of parsimony. Using a dozen different datasets, in MacCoun (2012) I compare the fit of the BOP models to various competitor models in the social psychology and social diffusion literatures, and show that the models provide a unifying framework for integrating research in the conformity, deliberation, helping, and social imitation/diffusion paradigms (see Fig. 1).

Points of convergence. Though they are parameterized somewhat differently, BOB and BOP are each variations on standard discrete choice models in the Luce/McFadden tradition, providing foundations in psychophysics, psychometrics, and econometrics. BOB's intensity parameter ( $b_{t}$, the "transparent-opaque" dimension) is similar to BOP's "clarity" dimension $(c)$. Both are inversely related to the standard deviation of the choice arguments. And BOB's "strength of influence" parameter $\left(J_{t}\right)$ appears to be very similar to BOP's "ceiling" parameter $(m)$.

Reasoning from the $\mathrm{BOB}$ map, Bentley et al. suggest that "(t)here are numerous indications that online behavior may be getting more herdlike" (target article, sect. 1, para. 5). Along similar lines, in MacCoun (2012) I use agent-based BOP modeling to offer a similar hypothesis, showing how herdlike behavior emerges as a result of an additional "vision" parameter representing the proportion of the total population whose views the agent is able to monitor. I speculate that web technologies combined with relentless polling is leading to dramatic expansions in vision.

Points of divergence. BOP decomposes the random utility model differently than $\mathrm{BOB}$, creating a threshold parameter. This is the heart of the BOP model, because the threshold parameter allows one to assess asymmetric influence - the extent to which one side of an issue "holds the burden of social proof." The threshold parameter allows BOP to capture the essence of both the Schelling (1969) tipping point model (when $b=.50$ and clarity is high), and the Granovetter (1978) distributed thresholds model (any $b$, when clarity is low). And it allows the BOP map to provide explicit theoretical landmarks - Proportionality, Simple Majority, 2/3 Majority, Truth Wins, Truth-Supported Wins - as a point of comparison for empirical estimates (see Fig. 1).

Figure 2, from ongoing meta-analytic work, provides an illustration. (See supplementary appendix for data sources.) Plotted using BOP's coordinates, studies of intellective tasks (for which there is a demonstrably correct answer relative to some conceptual scheme) and studies of criminal jury deliberations appear to form two distinct "continents." Both are a bit "north" and well "east" of the "proportionality" landmark. Both continents have asymmetrical thresholds, in which one faction bears a larger "burden of social proof." The jury data has more asymmetry than one would expect by simple majority influence, apparently due to the reasonable doubt standard (see Kerr \& MacCoun 2012). The intellective task data has greater asymmetry yet falls short of the "truth wins" landmark where a group will solve a problem if at least one member proposes the solution.

The BOP parameter space could also include the ceiling parameter (akin to BOB's "strength of influence" parameter). But only three of the several dozen datasets I've fit so far required such a parameter. Why? Partly because of sample selection, but it may be that even trivial individual decisions are susceptible to some social influence.

Attempts to fit BOP's clarity parameter imply that the scaling of BOB's $b_{t}$ from 0 to positive infinity may be misleading; in practice, the parameter has little detectable qualitative effect above about $\log _{10}(c)=2.5$. This seems reassuring; no one wants to embark on a journey carrying an infinitely large map. Still, Bentley et al.

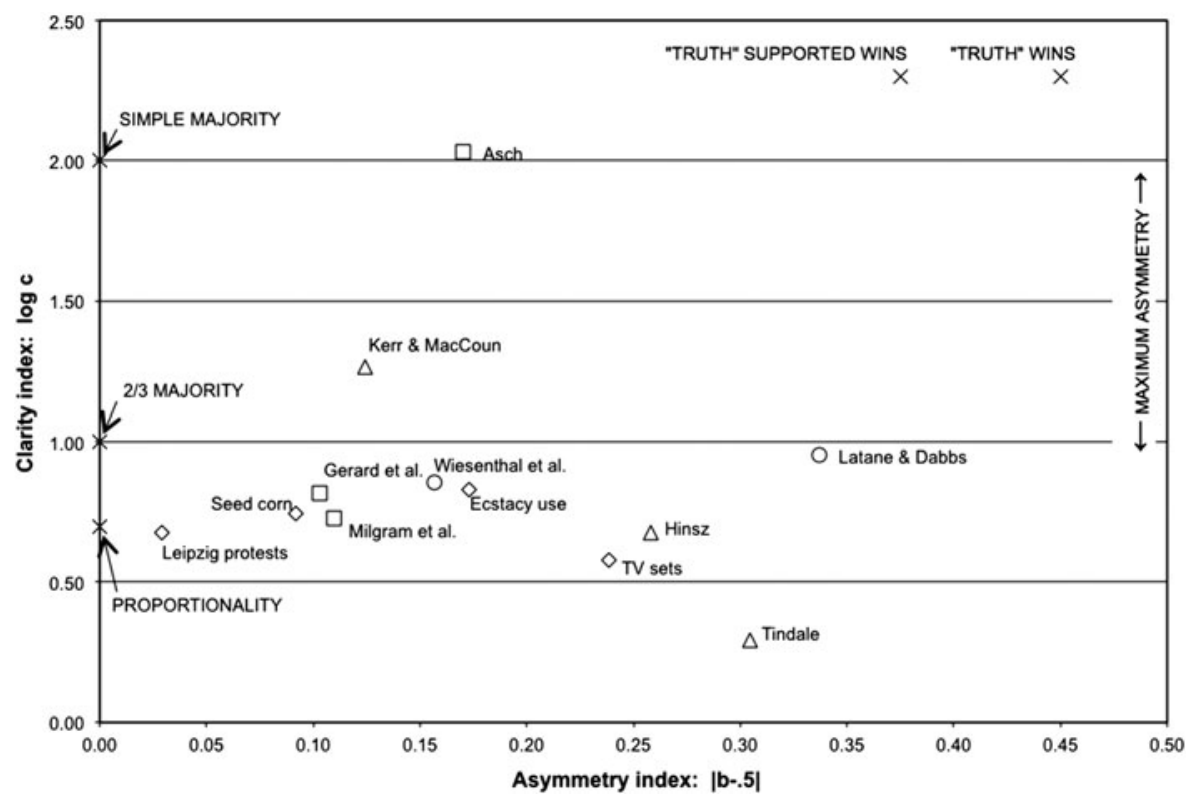

Figure 1 (MacCoun). Locating parameters from 12 social influence data sets in the BOP parameter space. $\square=$ conformity paradigm; $\mathrm{O}=$ helping paradigm; $\Delta=$ deliberation paradigm; $\diamond=$ social imitation paradigm; $x=$ theoretical social decision scheme landmarks. Source: MacCoun (2012, Fig. 11). 


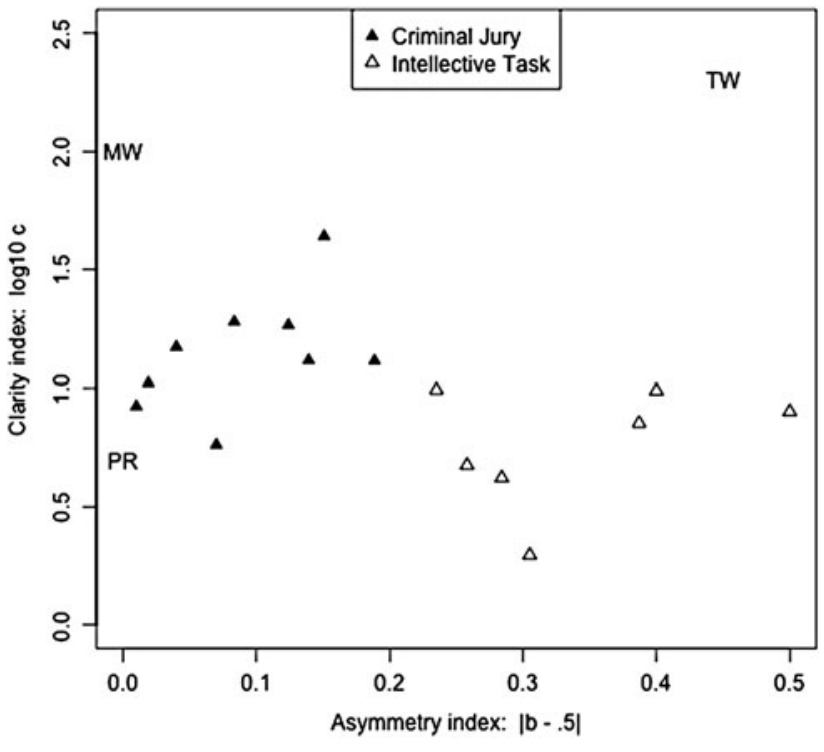

Figure 2 (MacCoun). Plotted BOP parameters for nine criminal jury studies $(\boldsymbol{\Delta})$ and seven intellective-task studies $(\Delta)$. Theoretical landmarks: $\mathrm{PR}=$ Proportionality decision scheme, $\mathrm{MW}=$ Majority Wins decision scheme, TW $=$ Truth Wins decision scheme.

offer intriguing arguments that qualitatively different models may be required in distinct regions of parameter space.

Conclusions. According to Monmonier (1996, p. 1), "not only is it easy to lie with maps, it's essential. To portray meaningful relationships ... a map must distort reality." In time, we will learn more about any distortions created by the BOB and BOP maps. Most but not all of the plotted data points in Figures 1 and 2 come from controlled experiments. But as we journey out into the deeper waters of big data, our parameter estimates will be increasingly susceptible to bias due to spurious correlations and causal endogeneity (see MacCoun et al. 2008). So our explorations promise new discoveries, but for now we might annotate our maps with the ancient warning: "Here be dragons."

\section{SUPPLEMENTARY MATERIALS}

To view supplementary material for this article, please visit http:// dx.doi.org/10.1017/S0140525X13001787.

\section{Coordination games, anti-coordination games, and imitative learning}

\author{
doi:10.1017/S0140525X13001799
}

\section{Roger A. McCain and Richard Hamilton}

Department of Economics and International Business, Drexel University, Philadelphia, PA 19104; Department of Emergency Medicine, Drexel University College of Medicine, Philadelphia, PA 19102.

mccainra@drexel.edu Richard.Hamilton@drexelmed.edu http://faculty.lebow.drexel.edu/mccainr/ https://www.drexelmed.edu/Home/AboutOurFaculty/RichardHamilton. aspx

Abstract: Bentley et al.'s scheme generates distributions characteristic of situations of high and low social influence on decisions and of high and low salience ("transparency") of rewards. Another element of decisions that may influence the placement of a decision process in their map is the way in which individual decisions interact to determine the payoffs. This commentary discusses the role of Nash equilibria in game theory, focusing especially on coordination and anti-coordination games.
Bentley et al. adapt a well-known probabilistic model of discrete choice to generate distributions characteristic of situations of high and low social influence on decisions and of high and low salience ("transparency") of rewards, to support inference from "big data" in which the details of individual decisions may not be recorded but the distributions are. Another element of decisions that may influence the placement of a decision process in their map is the way in which individual decisions interact to determine the payoffs. This is the subject matter of non-cooperative game theory (Aumann 2003; Schelling 1960). We consider three classes of non-cooperative games that, in a context of boundedly rational learning but with given parameters of social influence and reward salience, yield very different positions in the Bentley et al. map. The three classes of games are coordination games, anti-coordination games, and congestion games. We focus here mainly on anti-coordination games, but will refer to our own study of the determinants of congestion of hospital emergency rooms (McCain et al. 2011) to illustrate the point, with evidence from a questionnaire study that the expectations of the patients are consistent with a Nash equilibrium, based on learning from individual experience with some errors (thus in the far northeast of Bentley et al.'s map.) This poses the deeper question, at which Bentley et al. hint: How do individuals learn how best to learn from their own experience and the experience of others?

Anti-coordination games can be illustrated by an example from street traffic. Two cars meet, crossing, at a street intersection. Each has two strategies: to wait or to go. The payoffs are shown in Table 1. As we see, if both stop, they simply reproduce the problem, for payoffs of zero; but if both go, they will crash, for payoffs of -100 . If one goes and the other waits, the one who goes "wins," getting through the intersection first, for 5, while the other goes through the intersection second (but safely) for a payoff of 1 .

For this game, there are two Nash equilibria, each of the strategy pairs at which one car waits and the other goes. In this game, it is necessary for the players to choose different strategies in a coordinated way, in order to realize a Nash equilibrium, and while they are not equally well off at the equilibrium, both are better off than they will be at a non-equilibrium strategy pair. In some recent writing on game theory, games such as this are called anti-coordination games. The decision-makers will need a bit of information from outside the game in order to appropriately coordinate their strategies; but for an anti-coordination game, it is necessary that each gets a different bit of information, that can signal one to go and the other to stop. One possible source of this information could be a stoplight at the intersection. In general, however, imitative learning will not provide the kind of differentiated signal provided by a stoplight, and consequently anti-coordination games present a deeper problem for mapping in Bentley et al.'s map than do coordination games. This can be verified by agent-based computer simulation.

Congestion games share some of the characteristics of anticoordination games. Our study of emergency room congestion modeled the decision to seek emergency room service as a congestion game. To further extend the model and allow for (1) much larger numbers of potential patients; (2) heterogeneity of health states, experience, and expectation; (3) boundedly rational learning; and (4) initialization effects, dynamic adjustment, and transients, we undertook agent-based computer simulation. (Holland \& Miller 1991). We then validated the predictions

Table 1 (McCain \& Hamilton). The Drive On Game

\begin{tabular}{llcc}
\hline \hline & \multicolumn{2}{c}{ Mercedes } \\
\cline { 3 - 4 } & & wait & go \\
\hline Buick & wait & 0,0 & 1,5 \\
& go & 5,1 & $-100,-100$ \\
\hline \hline
\end{tabular}


of the computer simulation by a questionnaire study of patients in the emergency department of Hahnemann Hospital, associated with the Drexel University College of Medicine.

The key point for the Bentley et al. map is that learning in the simulations reported, although based on a probabilistic choice model such as Bentley et al. postulate, relied only on individual experience to learn and estimate the benefits from the different strategies available to the agents. Different individual signals from the individual's own past experience provide the different signals necessary to support a Nash equilibrium in an anti-coordination game. Thus, these simulations belong in the extreme northeast of the Bentley et al. map. Indeed we do see, as predicted, an $r$-shaped adoption curve. However, in preliminary simulations that did assume social learning, no tendency to converge to a Nash equilibrium was observed. The shared average experience of each type of agent provides no different signal that could support the choice of different strategies in the congestion game, as a Nash equilibrium requires in such a game. These simulations also, consequently, disagree with the evidence from the questionnaire study, which were consistent with the hypothesis of Nash equilibrium.

Here is the conclusion. On the one hand, the game-theoretic discussion and the agent-based computer simulations indicate that social learning is inappropriate to congestion games, in that it cannot provide signals that lead different agents to choose different strategies, whereas a reliance on individual experience may do so. On the other hand, the evidence suggests that the Nash equilibrium hypothesis is descriptive of the actual experience of patients in emergency departments. Somehow, people seem to have focused their attention and learning on the sort of information that could give rise to individually satisfactory outcomes, so far as the "game" permits. Perhaps the Bentley et al. map, which seems to take the social or individual bias of decision-making as given, needs some refinement on the basis of non-cooperative game theory.

\section{Cultural evolution in more than two dimensions: Distinguishing social learning biases and identifying payoff structures}

\section{doi:10.1017/S0140525X13001805}

\section{Alex Mesoudi \\ Department of Anthropology, Durham University, Durham DH1 3LE, United Kingdom. \\ a.a.mesoudi@durham.ac.uk \\ https://sites.google.com/site/amesoudi2/}

\begin{abstract}
Bentley et al.'s two-dimensional conceptual map is complementary to cultural evolution research that has sought to explain population-level cultural dynamics in terms of individual-level behavioral processes. Here, I qualify their scheme by arguing that different social learning biases should be treated distinctly, and that the transparency of decisions is sometimes conflated with the actual underlying payoff structure of those decisions.
\end{abstract}

The target article by Bentley et al. provides an innovative conceptual map of human decision-making in a social context. It may well provide a valuable guide to researchers who are beginning to analyse "big data", such as aggregate online purchasing decisions or peer-to-peer social interactions. I see their scheme as complementary to calls from myself and others (Gintis 2007; Mesoudi 2011; Mesoudi et al. 2006; Richerson \& Boyd 2005) to restructure the social and behavioral sciences around an evolutionary framework. Evolutionary "population thinking" concerns the exact problem that is addressed throughout the target article: how individual-level processes aggregate to form population-level patterns. In biology, the individual-level processes are natural selection, genetic mutation, Mendelian inheritance, and so on, and the population-level patterns include adaptation, speciation, adaptive radiation, serial founder effects, etc. Several decades of research has identified equivalent (but often different) individual-level processes in cultural evolution (Boyd \& Richerson 1985; CavalliSforza \& Feldman 1981; Mesoudi 2011), such as conformist- or prestige-based social learning biases (Boyd \& Richerson 1985), or the non-random generation of new cultural variation according to content-based inductive biases (Griffiths et al. 2008). Major advances in the social sciences can be made by borrowing tools from evolutionary biology to both explore the population-level consequences of these individual-level biases (e.g., populationgenetic-style mathematical models; see Bentley et al. 2004), and quantitatively identify and measure those population-level patterns in real cultural datasets (e.g., phylogenetic methods; see O’Brien et al. 2001).

The conceptual map presented in Figure 1 of the target article similarly links individual-level decisions (the degree to which individuals rely on social or asocial learning, represented by the east-west axis) made within different environments (the transparency in payoffs, represented by the north-south dimension) to different population-level patterns, such as the popularity distributions shown in Figure 2. While recognising the heuristic value in such a simple conceptual map, I caution that these particular dimensions may over-simplify and obscure some key issues, which the cultural evolution literature has identified in recent years as being particularly important for understanding cultural change.

First, regarding the east-west axis, it seems problematic to treat all social learning as equivalent, or at least as having broadly similar population-level consequences. Different social learning biases, such as the aforementioned conformist, prestige and inductive biases, may have very different population-level signatures. Models suggest that prestige bias can generate a runaway process towards maladaptively extreme values, while conformity generates particularly pronounced within-group behavioral homogeneity (Boyd \& Richerson 1985). Culturally driven copycatsuicide clusters require a particular combination of social learning processes in order to occur, primarily the rapid one-to-many transmission characteristic of the mass media plus a celebritydriven prestige bias (Mesoudi 2009). Even restricting ourselves to the popularity distributions that Bentley et al. focus on, Mesoudi and Lycett (2009) showed that conformity and anti-conformity have very different consequences on population-level frequency distributions of discrete traits such as first names, with conformity creating a "winner-take-all" distribution where popular traits are made even more popular, and anti-conformity favoring traits of intermediate frequency. In sum, knowing that social (as opposed to individual) learning is at work might be useful, but knowing what kind of social learning is operating seems to be crucial, too.

Second, the north-south "transparent-opaque" dimension appears to conflate feedback error in agents' decisions with the actual payoff structure that underlies different decisions. The north-south dimension is said to represent "the extent to which there is a transparent correspondence between an individual's decision and the consequences (costs and payoffs) of that decision" (target article, sect. 2, para. 1). In other words, it concerns feedback error: high feedback error equals opaque decision-making, while low feedback error equals transparent decision-making. This is captured formally in the $b_{t}$ parameter of equation 1. Yet this does not address the actual payoff functions underlying different choices (denoted by the function $U$ in the equation, but unaddressed in the map).

With respect to actual payoffs, there are several possibilities: There may be a single objectively best option and many bad options, or there may be several equally good options, or there may be no functional correspondence between choice and payoff whatsoever. In adaptive landscape terms (Wright 1932), these correspond to a unimodal, a multimodal (or rugged), and a flat landscape, respectively. The shape of this underlying 
adaptive landscape is logically independent to how well that payoff structure can be perceived by agents (i.e., the vertical transparency-opaqueness dimension).

I would argue that one cannot understand the consequences of transparent versus opaque feedback error without also considering the actual shape of the underlying adaptive landscape. Opaque feedback in a flat (neutral) landscape will be unproblematic, because all options are equivalent and feedback error is unimportant. However, opaque feedback in a rugged landscape will be very problematic, given the need to find one of a small number of fitness peaks. Conversely, perfectly transparent feedback may be problematic in a rugged landscape because it may lead learners to locally optimal but globally sub-optimal peaks/ decisions, whereas the error intrinsic in slightly opaque feedback might lead learners, by chance, off their sub-optimal peak and onto a higher peak elsewhere in the landscape.

Experiments and models show that the shape of the adaptive landscape can significantly affect both people's choices and the aggregate outcome of those choices, quite independently of feedback error (Mesoudi 2008; Mesoudi \& O'Brien 2008a; 2008b). Yet Bentley et al. appear to conflate these two distinct dimensions. For example, the neutral models discussed in section 2.3.1 (and analysed in Bentley et al. 2004) surely concern the case where the actual payoffs of all possible choices are equivalent, rather than where payoffs are opaque.

Naturally, all heuristic schemes such as the one presented by Bentley et al. are simplifications, and their value lies in that simplicity, as researchers grapple with the enormous datasets generated in the modern age. At the same time, oversimplification can sometimes lead to the wrong answer. I suspect that distinguishing between different social learning biases, and considering payoff structure as well as feedback error, might be crucial in avoiding those wrong answers.

\section{Using big data to predict collective behavior in the real world ${ }^{1}$}

\author{
doi:10.1017/S0140525X13001817
}

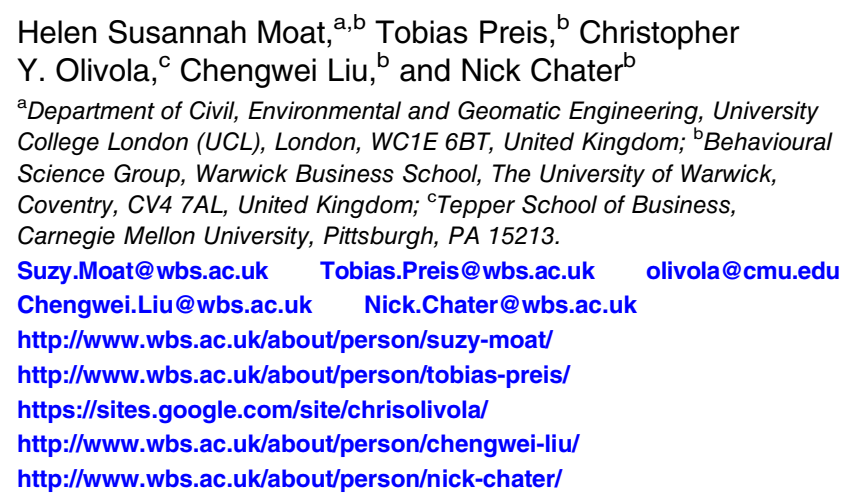

Abstract: Recent studies provide convincing evidence that data on online information gathering, alongside massive real-world datasets, can give new insights into real-world collective decision making and can even anticipate future actions. We argue that Bentley et al.'s timely account should consider the full breadth, and, above all, the predictive power of big data.

Modern everyday life is threaded with countless interactions with massive technological systems that support our communication, our transport, our retail activities, and much more. Through these interactions, we are generating increasing volumes of "big data," documenting our collective behavior at an unprecedented scale.

Bentley et al. provide a timely account of the role of big data in the study of collective behavior. They offer a comprehensive analysis of what our interactions on the Internet, in particular using social network sites such as Facebook and Twitter, can tell us about how information flows throughout the large and complex network of human society. While we agree that this insight into the structure of social connections is important, we emphasize that big data do not only come from online social networks. We note a number of recent studies providing evidence that big data can tell us much more about real-world collective decision making than has been acknowledged in Bentley et al.'s account, and can even allow us to better anticipate collective actions taken in the real world.

For example, human decision making often involves gathering information to determine the consequences of possible actions (Simon 1955). Increasingly, we turn to the Internet, and search engines such as Google in particular, to provide information to support our everyday decisions. Can massive records of our search engine usage therefore offer insight into the previously hidden information-gathering processes which precede realworld decisions taken around the globe? Recent results suggest that they can. A series of studies have shown that search engine query data "predict the present," providing a measurement of real-world behavior often before official data are released (Choi \& Varian 2012). Correlations between search engine query data and real-world actions have been demonstrated across a range of areas such as motor vehicle sales, incoming tourist numbers, unemployment rates, reports of flu and other diseases, and trading volumes in the U.S. stock markets (Askitas \& Zimmerman 2009; Brownstein et al. 2009; Choi \& Varian 2012; Ettredge et al. 2005; Ginsberg et al. 2009; Preis et al. 2010).

Further studies have illustrated that data on online information gathering can also anticipate future collective behavior. Goel et al. (2010) demonstrated that search query volume predicts the opening weekend box-office revenue for films, first-month sales of video games, and chart rankings of songs. Our own investigations have suggested that changes in the number of searches for financially related terms on Google (Preis et al. 2013) and views of financially related pages on Wikipedia (Moat et al. 2013) may have contained early warning signs of stock market moves.

In a recent study, we exploited the global breadth of Google data to compare information-gathering behavior around the world. Our analysis uncovered evidence that Internet users from countries with a higher per capita gross domestic product (GDP) tend to search for more information about the future rather than the past (Preis et al. 2012). For 45 countries in 2010 , we calculated the ratio of the volume of Google searches for the upcoming year ("2011") to the volume of searches for the previous year ("2009"), a quantity we called the "future orientation index." We found that this index was strongly correlated with per capita GDP. In ongoing work, we seek to better understand whether these results reflect international differences in decision-making processes. Perhaps, for example, a focus on the future supports economic success.

Aside from search data, other research has provided evidence that the massive datasets generated by our everyday actions in the real world can also support better forecasting of future behavior (King 2011; Lazer et al. 2009; Mitchell 2009; Vespignani 2009). Large-scale datasets allow us to look for patterns in collective behavior which might recur in the future, similar to the way in which we as individuals rely on the statistical structure we have observed in the world when trying to forecast consequences of decisions (Giguère \& Love 2013; Olivola \& Sagara 2009; Stewart 2009; Stewart et al. 2006). For example, analysis of data collected through daily police activities has shown that the occurrence of a burglary results in a short-term increase in the probability that another burglary will occur on the same street, with implications for behavioral models of how these crimes are committed (Bowers et al. 2004; Johnson \& Bowers 2004; Mohler et al. 2011). Such insights have been captured in predictive policing systems which aim to deploy police to areas before an offence 
occurs, with initial evaluations demonstrating a reduction in levels of crime (Johnson et al. 2007). Similarly, large-scale data on both long-distance travel by air and local commuting can improve predictions of human travel behavior and therefore the spread of epidemics, with clear consequences for the distribution of health resources such as vaccines (Balcan et al. 2009; Tizzoni et al. 2012).

When considered at greater breadth, we argue that, in contrast to Bentley et al.'s conjecture, big-data studies do far more than "allow us to see better how known behavioral patterns apply in novel contexts" (target article, sect. 4, para. 13). Online search data, for example, offer us insight into early information gathering stages of real-world decision-making processes that could not previously be observed, while large-scale records of real-world activity enable us to better forecast future actions by allowing us to identify new patterns in our collective behavior. Such predictive power is not only of theoretical importance for behavioral science, but also of great practical consequence, as it opens up possibilities to reallocate resources to better support the well-being of society. Our ability to extract maximum value from these datasets is, however, highly dependent on our ability to ask the right questions: a task for which experts in more "traditional behavioral science" (sect. 4, para. 13) are ideally placed.

\section{ACKNOWLEDGMENTS}

Helen Susannah Moat, Tobias Preis, and Nick Chater acknowledge the support of the Research Councils U.K. Grant EP/K039830/1, and Moat further acknowledges support from EPSRC grant EP/J004197/1. In addition, Moat and Preis were supported by the Intelligence Advance Research Projects Activity (IARPA) via Department of Interior National Business Center (DoI/NBC) contract number D12PC00285.

\section{NOTE}

1. Disclaimer: The views and conclusions contained herein are those of the authors and should not be interpreted as necessarily representing the official policies or endorsements, either expressed or implied, of IARPA, DoI/NBC, or the U.S. Government.

\section{The missing dimension: The relevance of people's conception of time}

\author{
doi:10.1017/S0140525X13001829
}

\section{Sarah H. Norgate, ${ }^{a}$ Nigel Davies, ${ }^{\mathrm{b}}$ Chris Speed,${ }^{\mathrm{c}}$ Tom Cherrett, ${ }^{\mathrm{d}}$ and Janet Dickinson ${ }^{\mathrm{e}}$}

${ }^{a}$ School of Health Sciences, University of Salford, Greater Manchester M6

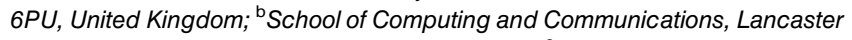
University, Lancaster LA1 4YR, United Kingdom; ' Edinburgh College of Art, The University of Edinburgh, Edinburgh EH3 9DF, Scotland, United Kingdom; ${ }^{\mathrm{d}}$ Transportation Research Group, University of Southampton, Southampton S017 1BJ, United Kingdom; ' $S$ School of Tourism, Bournemouth University, Bournemouth BH12 5BB, United Kingdom.

S.H.Norgate@salford.ac.uk nigel@comp.lancs.ac.uk

c.speed@ed.ac.uk T.J.Cherrett@soton.ac.uk

JDickinson@bournemouth.ac.uk

http://www.seek.salford.ac.uk:80/pp.jsp?NorgateSarah1374

Abstract: While a timely conceptual innovation for the digital age, the "map" proposed by Bentley et al. would benefit from strengthening through the inclusion of a non-clock-time perspective. In this way, there could be new hypotheses developed which could be applied and tested relevant to more diverse societies, cultures, and individuals.

Central to Bentley et al.'s vision is a "map" that captures the essence of human decision-making around collective behaviour in the digital age. Key to their argument is the expectation that decision-making is shifting towards imitation or herding driven by popularity and without grounding in the knowledge of the benefits of the behaviour. To explain this phenomenon, Bentley et al. focus on economies replete with online communication where people inhabit an information ecology characterised by data overload in terms of both the number of sources of intelligence and the number of choices on offer.

We argue that the authors' characterisation of decision-making occurring under conditions of time constraint biases the map towards a "clock time" culture. We suggest that adopting the "map" as an empirical framework appropriate for hypothesis testing may be premature unless the signature of the behavioural pattern also takes into account temporal perspectives anchored by alternative established societal and/or individual relationships with time. Specifically, the alternatives that we consider include a focus on "event time" together with a broadening of orientation towards future time.

The distinction between clock time and event time has been made socio-historically, particularly in relation to the rise of clock-time culture associated with mass timetabling consequent on introduction of the first passenger train in England in the 1820s (Zerubavel 1982) and the subsequent industrial revolution in Western Europe. More-recent distinctions between clock and event cultures focus on different behaviour patterns associated with each tendency. For instance, in clock time, activities are typically completed one at a time in sequence. In contrast, in event time, the inclination is to shift to and fro across tasks without any clock-determined schedules. One notion is that each society displays an overriding "average" set of behavioural markers towards either clock time or event time, whilst individuals within the society/culture will also display their own tendencies (e.g., Hall 1959) which correspond with these societal norms to a lesser or greater degree. In this respect, whilst the United States and Western Europe are considered to focus on clock time, South or Central America, the Middle East, and South Europe are more event focused (Lindquist \& Kaufman-Scarborough 2007).

Although there is a risk of oversimplification in adopting any conceptual dichotomy, the narrative around differentiation between the notions of clock time and event time (Levine 1997) has been theorized around the concepts of "monochronicity" and "polychronicity" (Hall 1959), particularly in relation to workplace behaviour. Monochronicity refers to the tendency to colonize time with one activity at a time whilst polychronicity revolves around doing more than one activity simultaneously.

Treatment of time as an economic resource is a hallmark of clock time where decision-making takes place under time constraints. However, in the big-data era, clock time is not necessarily exclusively dominant in economies replete with online communication. For instance, consider Japan. With a pervasive online economy and around $80 \%$ of the population accessing the Internet (MMG [Miniwatts Marketing Group] 2013), the culture yet functions on a combination of clock and event time (Tsuji 2006). In addition, in rural India, there is a growing reliance on the mobile Internet, against a backdrop where the predominant mode is event time.

Further, recent evidence suggests that there are discernible differences in the way humans self-regulate (Avnet \& Sellier 2011). People with a "self-regulation" couched in an approach known as "prevention" engage in activity which reduces the number of errors until no further risks are discernible. In this way, they are internally guided, thereby aligning with an event time rather than an external temporal cue. In contrast, individuals characterised with a self-regulation approach known as "promotion" rely less on internal resources to determine when a job is done and instead prioritise external, temporal cues to ensure a timely completion of tasks.

Evidence from across social science converges to suggest that alternatives to clock time exist. Within the digital age, this has implications for decision-making processes. In terms of trends which may guard against a shift to the southeast (cf. target article, sect. 3.1, para. 4) or provide an alternative conceptualisation of this shift, studies of cultural differences in decision making reveal a number of findings which suggest that the tendency towards herd-like outcomes may be less likely once we take a temporal cultural perspective. 
A number of studies reveal cultural dimensions to decision making. For instance, in a dynamic simulation task called COLDSTORE, individuals in clock time cultures (e.g., United States, Germany) tend towards "adaptor-type" decision making compared with those from event cultures (e.g., India, Philippines, and Brazil) who engage in more "oscillator-type" decisionmaking characterised by taking the present situation into account (Güss \& Dörner 2011). Significantly, whilst the "map" proposed by Bentley et al. focuses on the role of the past and the present, adopting a cultural perspective would mean taking into account the work by Zimbardo and Boyd (1999) where people have a predominant disposition to a future time orientation. The map could be strengthened by reflecting those cultures which display a time orientation towards the future, which may mean that Bentley et al. have underestimated the extent to which those populations below the "equator" in the southeast quadrant of their map can perceive benefits. We know, for instance, from work on risk-taking that cultures with more hierarchical views underestimate risk because they defer to the expertise of experts when making choices (Dake 1991).

Of further relevance to the mechanism of imitation associated with herding, is the notion that increased imitation can be desirable. For instance, in the domain of sustainable transport, any trends towards the uptake of carbon reduced or carbon neutral transportation systems are liable to be viewed as worthy outcomes. In particular, the practice of making real-time traffic jams on highways visible to prospective commuters via personal or public display systems has the scope to discourage imitation of a "popular" (typically fossil fuelled) trip mode taken by previous commuters. On a project called Sixth Sense Transport (Norgate et al. 2013), we are eliciting imitation of pro-environmental behaviour through design of Apps (applications) intended to achieve this.

In conclusion, we would like to persuade Bentley et al. to extend their "map" with a cultural perspective that reflects alternative conceptions of human relationships with time, and would enable social scientists to devise new testable hypotheses in topical domains within society.

\section{Big data in the new media environment}

\author{
doi:10.1017/S0140525X13001672
}

\section{Matthew Brook O'Donnell, ${ }^{a}$ Emily B. Falk, ${ }^{a, b}$ and Sara Konrath ${ }^{\mathrm{b}}$ \\ ${ }^{a}$ Annenberg School for Communication, University of Pennsylvania, Philadelphia, PA 19104; 'Institute for Social Research, University of Michigan, Ann Arbor, MI 48104-1248. \\ mbod@asc.upenn_edufalk@asc.upenn.edu \\ skonrath@umich.edu \\ http://cn.isr.umich.edu \\ http://www.iPEARlab.org}

\begin{abstract}
Bentley et al. argue for the social scientific contextualization of "big data" by proposing a four-quadrant model. We suggest extensions of the east-west (i.e., socially motivated versus independently motivated) decision-making dimension in light of findings from social psychology and neuroscience. We outline a method that leverages linguistic tools to connect insights across fields that address the individuals underlying bigdata media streams.
\end{abstract}

Recent events, such as the Arab Spring and London riots, demonstrate how the new media environment has recast the way that ideas spread and news is created. The breadth of participation and engagement, alongside the immediacy and complexity of these interactions, subvert the traditional notions of newsmakers and -influencers, and leave a large digital footprint ripe for the tools of big-data analytics. Bentley et al. highlight the proliferation of big data and the need to contextualize these data.
They propose that social scientific methods can provide insight about individuals who comprise the populations under study, thereby making big data more meaningful. For example, knowing the health behaviors (e.g., smoking habits, obesity/ weight loss) of individuals one or two degrees of separation from a person in a directed local social/friendship network, predicts whether that person will exhibit the same behaviors (Christakis \& Fowler 2007). In other words, knowledge of local network structures can contextualize population data regarding behaviors. Bentley et al. provide a conceptual model with which to interpret big data and demonstrate the model's validity in the economics domain. We agree with the broad premise, and believe that insight and methods from social psychology and social cognitive neuroscience can further deepen our understanding and ability to leverage big data in broader contexts.

For example, the classification framework proposes an eastwest dimension wherein the western edge of decision-making is described as entirely independent, and the eastern edge as entirely socially based. However, a large body of literature in social psychology and social neuroscience demonstrates that these distinctions are not so clear-cut. Within the brain, neural systems implicated in the retrieval of self-related knowledge are also frequently employed in social cognition (Decety \& Sommerville 2003; Lieberman 2010). Likewise, examples from everyday life challenge the notion that independent thinking and social thinking are placed at opposite ends of a single scale. For example, are decisions based on computer algorithms recommending products or services (e.g., Pandora, Netflix, amazon. com) considered independent, because no other live actors are necessary? Or are they considered social, because the data are derived from hypothetical or past actors? The category "social" itself includes face-to-face social interactions and interactions across different types of media, with no distinction between sources of social influence. Nor is there a discussion of how the type of social influence may differ based on the psychological closeness or "overlap" between self and target individual. Closeness is known not only to affect receptivity to social influence, but also the neural systems involved in processing other-related information (Kang et al. 2010). Finally, when discussing human decisionmaking, the idea of "independence" is hard to clearly demonstrate; the human mind itself is social, even in the absence of actual or virtual social interaction partners. Imagined social interactions (e.g., about what others will think of us if we choose a particular product or enact a particular behavior) make it complicated to label a decision as truly independent versus social.

At the macro level, big data provides a record of a complex set of interactions and processes, with individuals creating and responding to social stimuli at each point. Bentley et al. utilize the science of complexity to describe and understand these systems. New combinations of tools are needed to incorporate insights from a wider range of social and biological sciences. As one example, we have begun to harness tools from computational linguistics to link individual psychology and neuroscience with population-level outcomes. Language samples can be obtained at individual, group, and population levels, as markers of individual differences and cognitive states (Pennebaker 2011), and also as carriers that spread ideas. We draw upon developments in sentiment analysis from natural language processing to link levels of analysis and to contextualize big data. Recent studies of online social networks, such as Twitter and Facebook, have applied such tools to richly-linked and socially-situated language data (Bakshy et al. 2011; O’Connor et al. 2010).

Falk et al. (2012) illustrate how these tools can link activity within individual brains to the broader spread of ideas. They describe results from a neuroimaging study where subjects were exposed to socially relevant stimuli with a goal of predicting the ideas' propagation. Automated linguistic analyses classified postfMRI-scan verbal responses into more or less positive evaluative sentiments. During initial idea encoding, there was greater activation in neural regions associated with self-related processing 
and social cognition (medial prefrontal cortex [MPFC], posterior cingulated cortex [PCC]), and greater memory encoding (hippocampus) was associated with more positive post-scan sentiments. More positive descriptions were associated exclusively with neural activity in the temporal parietal junction (TPJ), a region often linked to perspective taking (Saxe \& Kanwisher 2003). The implication of this latter finding for understanding the first stages of idea propagation (contagion or "virality") is that individuals may be socially motivated right from the moment they encounter a new idea or potential "meme." In other words, as we have noted here, even in the absence of others (the "independent" end of a classification scheme), we may rely heavily on assumptions of what others will think, feel, and believe as we take in new information and prepare to make it useful to others. The use of automated linguistic analysis to connect brain to behavior allows scaling from the first order (those exposed to the original idea), to the second (those exposed to word-of-mouth description from first-order individuals; Falk et al. 2013), and so on, providing novel insight regarding the underlying mechanisms involved in the spread of ideas (Berger \& Milkman 2012).

In sum, although we question the east-west dichotomy of the dimensions proposed by Bentley et al. we fully agree with the underlying premise that tools from a range of social science disciplines are needed to more deeply ground our understanding of big data. We have presented initial examples of how social psychological and neural findings might add different perspectives to the framework proposed, and how linguistic tools can link levels of analysis. Additional research within these fields will further expand our ability to contextualize big data in the new media landscape and beyond.

\section{Independent decisions are fictional from a psychological perspective}

\section{doi:10.1017/S0140525X13001830}

\section{Hans-Rüdiger Pfister ${ }^{\mathrm{a}}$ and Gisela Böhm ${ }^{\mathrm{b}}$ \\ alnstitute of Experimental Industrial Psychology, Leuphana University Lüneburg, 21335 Lüneburg, Germany; ${ }^{\text {b }}$ Faculty of Psychology, University of Bergen, 5015 Bergen, Norway. \\ pfister@uni-lueneburg.de gisela.boehm@psysp.uib.no \\ www.leuphana.de/en/hans-ruediger-pfister.html \\ http://www.uib.no/personer/Gisela.Boehm\#profil} making does not capture crucial differences in decision making. Independence is fictional, and social influences substantially permeate preference construction. A distinction between deliberate and intuitive decision making would be more useful, and the problem in the big-data era is deciding when it is better to rely on deliberation and when to trust one's intuitions.

Bentley et al. propose a two-dimensional map that aims to describe decision making in the big-data era. We believe that by analyzing how the big-data era - together with the omnipresence of the Internet and the increasing interconnectedness of people via social networks - may shape decision making, Bentley et al. have chosen a highly relevant and timely topic. However, we think that the east-west axis, which contrasts independent and socially influenced decision making, is not a suitable dimension to use in this context because it is not well grounded in the field of psychological decision research. We identify two shortcomings: First, the type of behavior that they are trying to classify is ambiguously defined. Second, the notion of independent decision making is not empirically tenable.

First, much of what Bentley et al. refer to as "decision making" is not decision making proper as the term is used in the pertinent literature on psychological decision-making research (Baron 2007;
Hardman 2009; Kahneman \& Tversky 2000; Koehler \& Harvey 2004) or behavioral economics (Camerer et al. 2004; Kahneman 2003; Loewenstein 2007). Most importantly, Bentley et al. confound decision making and learning, which is, surprisingly, a fact that they acknowledge themselves: "we blur the distinction between learning and decision making" (target article, sect. 2, para. 7). Psychologically, however, decision making and learning involve fundamentally different processes. A decision-making process anticipates the future, whereas learning generates our memories. The acts of anticipating the future (Gilbert \& Wilson 2007) and remembering the past (Loftus \& Pickrell 1995) are prone to systematic biases. Furthermore, human decision making is notoriously resistant to learning from experience (Brehmer 1980; Kahneman 2011), leading to a multitude of non-optimal choices. Many of Bentley et al.'s signature patterns are patterns of optimal behavior, such as microeconomic utility maximization or the ideal free distribution. Such patterns may emerge under very specific conditions (environmental regularity, constant feedback) but are usually not present in situations of individual choice. In fact, we think that a more appropriate mapping would include learning and decision making as orthogonal dimensions, with the learning axis contrasting basic operant conditioning at one end with socially mediated learning at the other end.

Second, the contrasting notions of independent versus socially influenced decisions seem ill-founded from a psychological perspective. Assuming an independent decision maker in Bentley et al.'s sense invokes the traditional model of a selfish utility-maximizing homo oeconomicus who is equipped with a set of fixed and immutable preferences. This model has been rendered untenable by accumulating empirical evidence (Akerlof \& Shiller 2009; Ariely 2008; Gilovich et al. 2002; Kahneman 2003, 2011; Loewenstein 2007). Human preferences, which are the primitive elements of decision making, are anything but fixed and stable. A major finding from behavioral decision research is that preferences are constructed rather than given and are highly dependent on the context. Studies on constructive processes (Lichtenstein \& Slovic 2006), as well as studies on preference reversals and framing effects (Kahneman \& Tversky 2000; Tversky \& Simonson 1993; Tversky \& Thaler 1990) have demonstrated that preferences are subject to a large variety of contextual factors. An example is the ubiquitous role of anchors and reference points in determining utilities (Kahneman \& Tversky 1979; Ordónez et al. 2000; Thaler \& Sunstein 2008); for example, by simply changing the reference point (the default) from opt-in to opt-out, the preference concerning organ donation can be changed by $40 \%$ in favor of donation (Johnson \& Goldstein 2003). In sum, preferences are always shaped by a multitude of contextual influences, many of which are social in origin.

If the western end of Bentley et al.'s east-west dimension cannot be adequately characterized as independent decision making, then we must ask what defines its opposite. Bentley et al. label the eastern pole as socially influenced decision making where people thoughtlessly copy what others do. But is this the opposite of independent decision making? We view this as just a special, though common, case of a constructed preference: In many situations, the modal behavior of others may serve as a natural reference point; and relying on what others do is just an instance of a fast and frugal heuristic (Gigerenzer et al. 2002). We believe that it is not so much social influence that characterizes the decision-making processes at the eastern pole-because social influence is also present at the western pole - but their more intuitive character.

What then could serve as a psychologically founded decisionmaking dimension? We suggest that any realistic model of human decision making should be based on the roles of affect and emotions (Damasio 1994; Loewenstein \& Lerner 2003; Pfister \& Böhm 2008; Zeelenberg \& Pieters 2006). Emotions shape human preferences, and they provide the crucial link between deciding and acting (Böhm \& Pfister 2000; Pfister \& Böhm 2008; Zeelenberg \& Pieters 2006). Furthermore, a look 
at the functional roles of emotions suggests that individual as well as social decisions can be mapped onto specific emotions (Pfister $\&$ Böhm 2008; 2012). Some emotions are simple immediate affective reactions, such as joy or disgust, whereas others are cognitively saturated, such as guilt or envy.

We suggest that decision making be represented by a dimension that runs from deliberatelemotionally complex to intuitive/ emotionally simple, a distinction emphasized in current dualsystem approaches (Evans 2008; Kahneman 2011). A decision maker may rely either on an effortful process of deliberation and reasoning or on intuitions. Both modes of decision making can be advantageous or misleading, depending on the circumstances (Gigerenzer 2007; Hogarth 2010). We speculate that in a big-data era, it will become a critical issue for decision makers to select the appropriate mode, as the two modes often conflict. An Amazon purchase recommendation may superficially conform to a person's intuitive preferences, but may be rejected after some deliberate reasoning. On the other hand, when facing an unmanageable number of options, a deliberate decision might not be feasible, as emphasized by Bentley et al., thus raising the issue of how to educate our intuitions to survive in times of limitless choices.

\section{What shapes social decision making?}

\section{doi:10.1017/S0140525X13001842}

\section{Simon M. Reader ${ }^{\mathrm{a}}$ and loannis Leris $^{\mathrm{b}}$ \\ ${ }^{a}$ Department of Biology, McGill University, Montréal, Québec, H3A 1B1, Canada; ${ }^{\mathrm{b}}$ Behavioural Biology, Department of Biology, and Helmholtz Institute, Utrecht University, $3584 \mathrm{CH}$ Utrecht, The Netherlands. simon.reader@mcgill.cari.leris@uu.nl http://biology.mcgill.ca/faculty/reader/}

\begin{abstract}
Outcome transparency and the weight given to social information both play important roles in decision making, but we argue that an overarching influence is the degree to which individuals can and do gather information. Evolution, experience, and development may shape individual specializations in social decision making that carry over across contexts, and these individual differences may influence collective behavior and cultural evolution.
\end{abstract}

Bentley et al. discuss how technological advances, particularly online connectivity, change human decision making and collective behavior and their empirical study. They usefully caution that although large-scale online data provide new opportunities for the understanding of human behavior, there are also new pitfalls. Bentley et al. present a deliberately simplified conceptual map of different types of human decision making, with two dimensions: the degree to which personal versus social influences shape a decision, and the transparency of the payoffs of a decision. We agree that these are important determinants of decision making, and the two dimensions provide a useful simplification for several applications. However, we would like to draw attention to other determinants of decision making, particularly the influence of past experience, individual differences, and payoff structure, all of which may affect large-scale patterns.

The weighting of personal experience ("individual information") against information provided from others ("social information") is a key determinant of human decision making, and numerous factors can determine this weighting, such as the predictability of the environment, the relative costs of social and individual information, or the availability of suitable models to learn from (Boyd \& Richerson 1985; Laland 2004). Frequently, individual and social information will together determine a decision (Kendal et al. 2009; Salganik et al. 2006). However, adaptive decisions can be made without gathering information (Dall \& Johnstone 2002; Stephens 1991), and thus the intensity of information use in a decision may predominate over whether individual versus social information is utilized. Important decisions in particular are likely to involve substantial use of both individual and social information. Based on work on animal personalities (e.g., Marchetti \& Drent 2000), we suggest that the amount of information gathered for decision making may consistently differ between individuals, with certain individuals more likely to utilize both individual and social information. That is, we predict individual and group differences in their sensitivity to information, in the readiness to search for information, as well as in the strategy used to obtain information.

Bentley et al. discuss several aspects of the transparency of decision payoffs, such as whether a right or wrong decision is immediately detectable, whether a payoff can be assessed before a decision is made, the equivalence of payoffs, the degree of understanding of the processes that determine payoffs, and the ease of assessing who to learn from. Since these aspects of transparency may not covary, we urge caution in treating payoff transparency as a unitary entity. This is particularly important because payoff transparency, together with the costs of decision making, are likely to determine whether social or individual information is utilized: When the best decision can easily be determined individually, social information will be less advantageous (Boyd \& Richerson 1985; Kendal et al. 2009). Thus, the two dimensions of Bentley et al.'s conceptual map are not independent.

Payoff transparency may depend on numerous additional characteristics, such as the way that competition or frequency dependence affect payoffs (McNamara \& Fawcett 2012), the shape of the "adaptive landscape," and thus the degree to which similar past decisions inform current decisions, and the kind of social information that models provide (Beppu \& Griffiths 2009; Rendell et al. 2011). Assessment of payoff transparency is further complicated since a single decision may have multiple, possibly conflicting, payoffs in different domains. For example, depending on circumstances, deviating from group behavior may have economic benefits but social costs, or, alternatively, independent innovation may be socially rewarded but economically costly (Day et al. 2001; Greve 2003). Bentley et al. argue that differences in agent competencies will not be visible at an aggregated scale. However, groups may differ in the way payoffs are assessed, potentially resulting in different processes in different groups. For example, children and adolescents differ in the way they assess losses and long-term rewards, compared to adults (Aite et al. 2012). Similarly, experts may outperform the "wisdom-of-the-crowd" depending on the task (Krause et al. 2010), meaning that individual competencies may have significant influence. A striking example of expertise trumping the wisdomof-the-crowd is the victory of top-ranked chess player Magnus Carlsen over a large online audience (referred to as "The World") that participated by choosing one of three available moves proposed by a group of chess grandmasters (McClain 2010).

We consider whether humans can switch freely between incorporating different kinds of information in their decisions. Each decision could be optimized by determining which informationgathering strategy to apply, which may itself rely on social cues (Toelch et al. 2011). However, the costs of assessing which strategy to employ may outweigh any achieved benefits, and prior knowledge and individual characteristics, perhaps in combination with evolved predispositions, could shape the strategy employed. Current, past, or early life experience can be used to determine which information-gathering strategy is most likely to be profitable. For instance, humans playing a computer game apparently used the degree of environmental variability as a heuristic cue for determining whether to copy others (Toelch et al. 2009). In nonhuman animals, recent and early life-experience determines the reliance on social information (Chapman et al. 2008; Dawson et al. 2013; Katsnelson et al. 2008; Lindeyer et al. 2013). Thus, experience and development may influence future decision making, resulting in carryover effects where experience with one problem type or in one domain influences the decision 
making employed for other problems. Indeed, individuals may specialize in particular information-gathering strategies.

The opportunities and pitfalls provided by "big data" increasingly apply to other fields that have traditionally utilized relatively small datasets and direct behavioral observations, such as animal behavior and behavioral neuroscience (Sumpter et al. 2012). It is now possible to track groups of free-living animals, their interactions, their physiology, and their neural activity for long periods (Cavagna et al. 2010; Dell'Omo et al. 2000; Krause et al. 2011; Vyssotski et al. 2009). While automated recording and testing provide unprecedented opportunities to monitor the behavior of multiple individuals, the use of empirical population-level signatures as indicators of learning processes must be validated (Reader 2004), rare behavior patterns should not be overlooked (Krause et al. 2011), and, ideally, direct observations should ensure that the relevant behaviors are indeed recorded. For example, electronically tagged mice adopted a group-foraging strategy and thus circumvented an automated system programmed to reward particular individual foraging patterns, a development easily identified by video observations of behavior (Dell'Omo et al. 2000). Large datasets cannot substitute for well-designed studies, but appropriate use of "big-data" methodologies provides novel and valuable tools for the study of human and nonhuman animal social behavior alike.

\section{ACKNOWLEDGMENTS}

We gratefully acknowledge funding by Utrecht and McGill Universities and by the Natural Sciences and Engineering Research Council of Canada (NSERC).

\section{Bigger data for big data: From Twitter to brain-computer interfaces}

\section{doi:10.1017/S0140525X13001854}

\section{Etienne B. Roesch, ${ }^{\mathrm{a}, \mathrm{b}}$ Frederic Stahl, ${ }^{\mathrm{a}}$ and Mohamed Medhat Gaber ${ }^{\mathrm{C}}$}

aschool of Systems Engineering, University of Reading, Reading RG6 6AH, United Kingdom; ${ }^{\mathrm{b}}$ Centre for Integrative Neuroscience and Neurodynamics, University of Reading, Reading RG6 6AH, United Kingdom; ' School of Computing Science and Digital Media, The Robert Gordon University, Aberdeen AB25 1HG, United Kingdom.

contact@etienneroes.ch

http://etienneroes.ch

f.t.stahl@ reading.ac.uk

http://fredericstahl.wordpress.com

m.gaber1@rgu.ac.uk

http://mohamedmgaber.weebly.com/

Abstract: We are sympathetic with Bentley et al.'s attempt to encompass the wisdom of crowds in a generative model, but posit that a successful attempt at using big data will include more sensitive measurements, more varied sources of information, and will also build from the indirect information available through technology, from ancillary technical features to data from brain-computer interfaces.

Recent developments in the area of "big data" lay the ground for exciting new avenues to understand human behavior on a large scale, as demonstrated by Bentley et al.'s impressive data-driven classification scheme. When they rightfully emphasize the magnitude of "BIG data" (their emphasis; target article, sect. 4, para. 13), we posit that what should be aimed at is really BIGGER data, and that, as they acknowledge, the dimensions they investigate, social influence $\left(J_{t}\right)$ against transparency of payoff $\left(b_{t}\right)$, constitute only part of the picture that makes for the process of human decision making. The importance of these factors for decision making is unquestioned, but a successful attempt at using the full potential of big data will study this process over time, will go beyond Twitter and Facebook to take into account technological advancements that are pervasive and constitutive of our modern societies, and will build on more various types of information, spanning second-order, indirect information as well as more personal data - from the brain sciences, for instance.

The maps presented provide a static representation of the decision-making process of a given society, at a particular point in time. Provided that such data can be gathered, Bentley et al. venture to comment on the evolution of societies and communities over time, asking whether human decision making is generally drifting to a more social, yet more opaque, mode of functioning. In our opinion, complementarily to this analysis, one could use the velocity of change of decisions/opinions, to map the intrinsically dynamical nature of human societies, based on the distance covered between two maps at fixed time intervals.

Much in line with the dynamics observed in Kuhnian paradigm shifts (Brock \& Durlauf 1999), recent events in the wake of political turmoil in the Arab world - for instance, the so-called Arab Spring-showed (again) that societies have the potential to impose radical changes to their way of functioning. The exponential velocity with which this change occurs is indicative of the multiple and complex tensions that arise in human societies. The speed of change will undoubtedly be impacted by access to new technology, liberal versus conservative cultures and policies, trait volatility (cultural propensity to changing one's mind), and will provide additional information about the importance of a given decision/opinion state, represented by Bentley et al.'s map, at any point in time. Furthermore, one could imagine that this velocity could signal imminent change, with sufficient predictive power to serve as a tool for monitoring, and supporting, largescale decision making.

Technology plays an unequivocal role in shaping human societies. Big data, in fact, spawns from the almost unmanageable sea of information generated from modern technology, which forms the scaffolding for our daily activities. The ubiquity of this technology pervades our lives to such an extent that individuals can participate in the flow of information instantly, at their leisure, from almost anywhere in the world, through the touch of a fingertip. Therefore, we envision big data to evolve toward a more holistic perspective of collective behavior, not only based on the opinions and the so-called wisdom of the crowd, accessible through social networks, for instance, but also to reflect more indirect sources of information, and to combine varied types of information.

The mining of the data generated by technology usage itself, for instance, can be a very rich source of such data. As mobile computing grows cheaper, easier to use, and more context-aware, as well as more anchored and necessary in our modern cultures, it is likely to become a prime source of inspiration for the field of big data. This mining process, which we call Pocket Data Mining, endeavors to extract information from the stream of data that is processed and emanates from users' devices (Stahl et al. 2010). This data may contain environmental variables, like temperature, noise level, luminance information, or energy consumption. It may not even refer to the actual content of these streams of data but reflect networking constraints, triangulated location information, or simply the sheer numbers of devices or the volume of communication.

Indirect big-data information will also come from the technical side of social networks, which comprises mining and consolidating strategies to cross-reference users' behavior. These strategies constitute prime features of e-commerce, such as recommendation systems (Bhasker \& Srikumar 2010), which go beyond the more direct social links intentionally set up by users on Twitter or Facebook, and the exchange of status update several times a day. Recommendation systems in retail applications, such as Amazon, are often used for purposes of advertising to potential customers, who may share similar preferences. It is important to note that there is neither direct communication nor explicit interaction between these users. Yet, they indirectly influence each other by their own decision making, due to the imaginative processes at play 
to make a business more lucrative. This dimension will no doubt play an even bigger role in the way we make decisions in the years to come as such features become deeply engrained in the most basic services we use daily - think of the impact that the order of Google results has on one's daily decision-making process, for instance.

Finally, we believe that technology will not stop at our fingertips, and neither should big data. As Google Inc. (2012) has just unveiled its head-mounted augmented reality display Project Glass, and the off-the-shelf brain-computer interface market is booming (Ebrahimi et al. 2003), it is increasingly likely that more personal information will be made available through technology sooner rather than later. If the practical consequences of such technology are difficult to predict, be they good or bad, it is yet entirely possible that this novel source of information will feed into our decision-making process. By that time, Twitter and Facebook may have been shown to reveal only the tip of the iceberg on what really pushes us to make decisions.

\section{Economics is all over the map}

\section{doi:10.1017/S0140525X13001866}

\section{Don Ross}

School of Economics, University of Cape Town, Private bag, Rondebosch
7701, Cape Town, South Africa.
don.ross@ uct.ac.za
http://uct.academia.edu/DonRoss

Abstract: Bentley et al. say that economics is the science of their map's northwest quadrant, where choice is individual and transparent. This accepts the picture of the discipline common among behavioral economists who aim to drag economics southward but not eastward. In fact, leading economics journals regularly publish models located in all four quadrants, and the prominence of work from the eastern zone is increasing.

The distinction between decision-making by rational individual deliberation and decision-making by following socially constructed canals or by copying others is a main basis of alternative methodologies in the social and behavioral sciences. Following conventional wisdom and the standard individualistic rhetoric of many economists, Bentley et al. locate economics in the northwest quadrant of their two-dimensional map.

The value of Bentley et al.'s schema does not depend on associating disciplinary paradigms with quadrants. However, it is worth taking trouble to jam such associations, especially as the authors offer remarks that encourage them, at least with respect to economics. The idea that economists in general model decision-making or choice as individual and transparent reprises the caricature of the discipline promoted by behavioral economists. In fact, top economics journals publish modeling approaches inhabiting all four of Bentley et al.'s quadrants; and models from the eastern side of their map are steadily gaining in importance.

Before elaborating on this main point, one semantic caveat is needed. Bentley et al. refer to their subject matter as "decision making." Most economists would understand this to refer to processes modeled by formal decision theory, including decision theory that accommodates bounded rationality. On that usage, Bentley et al.'s contention that economists locate decisionmaking in the northwest quadrant becomes tautological. But Bentley et al. diverge from the economist's convention by saying that any decision-making occurs in other quadrants. In economists' language, Bentley et al.'s topic is choice, that is, the family of information-processing patterns that link changes in environmental contingencies to changes in agents' behavior at least partially by way of cognitive comparison of actual states, hypothetical states, and goal states. I follow this second convention here.
Behavioral economists who see themselves as leading a "paradigm shift” typically take methodological individualism for granted. That is, they suppose that models of economic phenomena on social or macroeconomic scales of representation and analysis are built from - aggregated from, in one formal sense or another-models of individual deliberation and/or neural processing. They then argue that traditional economists have exaggerated the logical transparency and consistency of individual choice, leading them to conflate real, empirical human choice with decision-making by a fictional ideal Homo economicus. In Bentley et al.'s terms, such critical behavioral economists then emphasize the importance of psychological framing influences, tolerances for inconsistencies, and conflicting motivations that tend to drag real choice in the southerly direction. (See Angner [2012] for a survey.) The rhetoric of some such theorists implies that most individual choice should be modeled as a southwest phenomenon (Ariely 2008). Most are more cautious, maintaining only that psychological influences on economic choices tend to generate substantial and thick tails in the southwest (Angner \& Loewenstein 2012).

Bentley et al. seem to endorse this picture. Echoing standard rhetoric of behavioral economists, they say that "[b]ecause Homo economicus is located at the extreme northwest, he does not appear to be the primary model for human culture" (sect. 4, para. 8). However, the behavioral economics literature tends to reflect unreliable history of economics. Homo economicus is much less important in the historical development of the technical apparatus of standard economic analysis than is alleged. Samuelson (1947), after analyzing $H$. economicus in one chapter of his classic book on theoretical foundations - arguably the most influential text in postwar economic theory-dismissed the construction as being of little working importance (p. 117), and gave it no role in later chapters. Samuelson synthesized a neoclassical tradition that emphasized consistency in choice with a Keynesian relaxation of informational transparency and an openness to acknowledgment of structural, market-scale constraints and influences on choice.

We are commonly told that the Samuelsonian synthesis unraveled in the 1970s and was displaced by a new modeling style that insisted on microfoundations for aggregate-scale (macroeconomic) models and based market-scale analysis on the behavior of a single "representative" agent who maximizes expected utility à la Savage (1954) using all available information, with the only concession sometimes made to Keynesian social influences being specific dynamic rigidities such as sticky prices. If this approach had in fact taken over economics, as its promoters often announced that it would or already had, then Bentley et al. would be correct to locate economics as a science of the northwest. But rational expectations models, with or without "New Keynesian" elements, never eliminated alternative approaches (Colander 1996; Frydman \& Goldberg 2007), and in the wake of the 2008-2009 financial disaster, they are in headlong retreat (Timbeau 2012).

The historically brief overemphasis on economic choice as paradigmatically individual and transparent has not merely passed in macroeconomics; it is also being pushed back in non-behavioral but firmly empirical, frequently experimental, microeconomics (Smith 2008). The liveliest source of modeling innovation in microeconomics concerns effects of agent heterogeneity, which gives rise to emergent structural parameters. A particularly promising such approach estimates maximum-likelihood mixture models that yield distributions of types at the population level and uses this as a basis for predicting effects of incentive changes on markets and other social structures. (See, for example, papers in Cox \& Harrison 2008.) Essentially, these are representative agent models with multiple representatives. The conditioning variables on which the representatives are clustered are demographic. Thus, the representatives should not be identified with individual people, and we find ourselves in Bentley et al.'s northeast quadrant. Models that accommodate heterogeneity can also be embedded in global games (Carlsson \& van Damme 1993; Morris \& Shin 2003), where agents often disregard and so fail to reveal their private information. Though players of 
global games are modeled as fully rational, exogenous noise can push them into Bentley et al.'s southeast quadrant, leading to such phenomena as market bubbles and bank runs. Finally, microeconomic modeling of labor, product, and technology diffusion markets increasingly borrows network theory (Goyal 2007), the dominant mathematics of the southeast.

There is irony here. Behavioral economists criticize standard economics as, in Bentley et al.'s terms, stuck in the northwest. They then creep cautiously south, while the economists they criticize range all over the map.

\section{Understanding social networks requires more than two dimensions}

\section{doi:10.1017/S0140525X13001878}

\section{Derek Ruths ${ }^{\mathrm{a}}$ and Thomas Shultz ${ }^{\mathrm{b}}$}

${ }^{a}$ School of Computer Science, Network Dynamics Lab, McGill University, Montreal, Quebec, H3A 2A7, Canada; ${ }^{\mathrm{b}}$ Department of Psychology, Laboratory for Natural and Simulated Cognition, and School of Computer Science, McGill University, Montreal, Quebec H3A 1B1, Canada.

derek.ruths@mcgill.ca thomas.shultz@mcgill.ca

http://www.derekruths.com/

http://www.tomshultz.net/

Abstract: The proposed framework is insufficient to categorize and understand current evidence on decision making. There are some ambiguities in the questions asked that require additional distinctions between correctness and accuracy, decision making and learning, accuracy and confidence, and social influence and empowerment. Social learning techniques are not all the same: Behavior copying is quite different from theory passing. Sigmoidal acquisition curves are not unique to social learning and are often mistaken for other accelerating curves.

Bentley et al. address the issue of how people make decisions in the realm of large-scale social media. They propose an interesting two-dimensional framework in which to classify major influences on decision making: an east-west axis reflecting the degree to which decisions are made individually versus socially influenced, and a north-south axis reflecting the degree of transparency of the payoffs and risks associated with particular decisions. This is a useful start, and Bentley et al. get a certain amount of mileage from considering decision making within this framework. However, it is already apparent to us that this framework is insufficient to categorize and understand current evidence on decision making.

The question being asked. An issue that overshadows the work is a lack of clarity as to what aspect of human decision making they want to approach. A model is best evaluated within the context of the question it is being used to answer. For example, two specific questions that seem relevant to the proposed framework are: (1) "How do social context and critical thinking interact in producing right or wrong thinking?" and (2) "What is the role of social engagement in decision making?" These questions are reasonable, but involve the use of the proposed model in different ways. Furthermore, neither can be answered using the framework as proposed: For case 1, the model lacks a notion of correctness; for case 2 , the model is unclear about modes of social interaction. In the target article text, the authors do not identify a particular, precise question that their approach addresses. This issue derives, in part, from ambiguity about how certain aspects of the proposed model should be interpreted. We highlight three of these below.

Decision making or learning? Despite their discussion of decision making in their introductory remarks, it is sometimes unclear which of these human processes Bentley et al. are applying their model to-decision making or learning. Although decision making and learning are related, they are distinct processes that cannot be lightly interchanged (Busemeyer \& Johnson 2006).
Accurate or confident perception? The proposed north-south axis is described in terms of clarity of information. But what is the nature of this clarity? Are we concerned with whether the individual feels confident about their perception of risks and rewards, irrespective of whether their perception is correct; or are we concerned with whether the individual accurately perceives the risk/ rewards? The two are not the same thing and may need to be separated into different axes.

Social influence or empowerment? The social (east-west) axis characterizes an ambiguous social dimension. Does moving east involve other people having increasing sway over what the individual believes (social influence) or other people having increasing sway over whether an individual acts on their belief (social empowerment)? Each of these is valid, but they reflect quite different and important aspects of decision making in a social context.

Lumping together all social learning methods. Bentley et al. lump a variety of social learning techniques together under a high value of their $J$ parameter. However, there is now evidence that cultural transmission, defined as theory passing, has sharply different characteristics from imitation (Montrey \& Shultz 2010). Imitation (defined as attempted copying of behavior) is a lossy way to transmit information and may quickly become outdated in rapidly changing environments. In contrast, cultural transmission via passing of theories from one agent to another builds on existing knowledge, creating a strong ratchet effect with very little backsliding. In the Montrey and Shultz study, there were three learning methods in an agent-based model on a fully occupied lattice: imitation, exploration, and theory passing. Each learning method was implemented as a variant of Bayesian learning. As in previous work (Beppu \& Griffiths 2009), three alleles were compared: imitation alone (least adaptive), imitation plus exploration (moderately adaptive), and imitation plus exploration plus theory passing (most adaptive). Agents reproduced by cloning an offspring according to their own fitness.

Shape of acquisition curve is not definitive. The authors use the shape of acquisition curves to unequivocally identify underlying learning strategies. But this method is known to be difficult and often unreliable because the same shape can be consistent with more than one learning method. For example, individual learning typically produces a sigmoidal curve (Shultz 2003), a shape that Bentley et al. assume is a unique signature of social learning. Not only is the overall acquisition curve often sigmoidal (basically, a spurt connecting two plateaus), but with denser time sampling, numerous sigmoidal spurts can be found. Moreover, a number of different accelerating functions have been successfully fit to social learning data (Reader 2004). Even if the underlying social learning curve is truly sigmoidal, it can be mistakenly viewed as accelerating if final data points are missed, or as decelerating if early data points are missed.

Closing remarks. Despite the concerns raised above, Bentley et al. meaningfully contribute on an important topic: how social, media, and internal milieus interact to inform human decision making. The problem is highly dimensional, and Bentley et al. have provided a framework on which future work can productively build.

\section{The global shift: Shadows of identifiability}

\section{doi:10.1017/S0140525X1300188X}

\section{Colin T. Schmidt}

Head of Technology Appropriateness, ENSAM-ParisTech \& Laval Technical Institute, LeMans University, 53020 Laval, France.

Colin.Schmidt@univ-lemans.fr

https://www.researchgate.net/profile/Colin_Schmidt/

Abstract: The presence of overwhelming amounts of information in our big-data era society is growing. Globalisation is increasingly giving these solicitations (regarding information) a more social aspect causing 
behavioural changes. While restricting my focus on this aspect of the Bentley et al article, I address related medical questions and pin-point the conceptual interest of the roadmap given therein.

Let's make the geography of the target article explicit. Bentley et al. begin to focus on geography with a schema in their section 2 entitled "The map." A schema is of interest here because, for example, people in Africa and Asia have highly collaborative ways of doing things. Europeans and Americans, however, are often independent-minded users of technology and discourse. The possibility of current language uses drifting towards collective-minded places in the world (like the southeast as suggested by Bentley et al.), is related to the digital shadows of a person. Being able to control one's digital shadow (meaning the implications and usage of one's profiles, avatars, memberships in social media, or general presence on the Web), with the appropriate discourse, whether digital or not, is important to inhabitants in the West. In spite of this current situation, the globalization of cultures and economies is bringing about changes to priorities with respect to language use as the West becomes exposed to the collective-minded southeastern cultures.

So what does this mean for the western populations? The ambient perseverance on the Internet of an increasing number of identities of a person (memberships, enrollments, sign-ups, connections) will enable chronic forms of fatality about the (possible) uses of one's identity to set in with respect to the information they provide in order to connect to services (some may totally give up controlling their virtual identities). Users must provide an identity each time they sign up for access to a new website. Recording takes place in an ambient manner. Sometimes regrettable past identities linger on. Many wish to avoid leaving such shadows behind. Mathematically, one may state that the big-data business of "cleansing" the web of individuals' digital shadows will slow down, but this does not mean that the types and methods of communication in the West will be "homogenised" with the mentality of the southeast. Or does it? If this happens over a long period of time, a rather unclear self-identifiability issue on the Internet should not throw a skew to the general overall evolution of the language base, meaning that language skills will rest intact. But as the relationship between people in the northwest and the southeast quadrants of the map is certainly dialogical, as in all human relations, the southeast will gradually acquire a double role: (1) that of subduing, through a global relativity, the problematic 'onslaught' of dealing with digital-shadows in the West, and (2) that of potentiating acceleration of the same problem through collective behaviours. Proper analysis of the phenomenon is required now (but it takes time to achieve it; cf. Alleva (2006)). In certain highly mediated cases (i.e., directors and officers of corporations being arrested, etc.), multiple clouds of shadow-like identities even "spew out" over the Internet. The work of Bentley et al. in this issue obviously makes a major contribution to clarifying matters by offering us a roadmap.

This said, my geographical suggestion above falls somewhat short of a full explanation of this aspect of the text even though the authors' idea of a great shift is also argued for in a spatio-temporal manner. Equally relative to the constructive map provided by the authors, I think the shift across territories is just as important conceptually to specialists in cognitive technologies, philosophical questions about society and biological psychology (Pillai et al. 2013; Schmidt \& Kraemer 2006; etc.). This would, in my view, explain their setting out to elaborate such an instrument of sociological and ethnographic interest. Their arguments lead them to point out the fact that the shift towards the customs and practices of the (conceptual) southeast causes "decision fatigue" to set in is very intuitive: "As the number of options grows, a natural way to try to minimize the cognitive cost of choosing among them would be to simply copy the choices of moreexperienced choosers" (cf. sect. 2, para. 4).

The cognitive constraints so expressed encouraged me to reflect upon what this meant for a person's 'normal' freedom of thought (cf. Canguilhem 1966). In the West, ethics and well-being are at stake here, or to put things plainly, one may speak of the mere employability of members of society that avoid thinking for themselves. Actually abandoning one's right to choose, pick, select, discern for oneself from one's own knowledge, and so forth, in order to adopt dubious methods of controlling one's situation is to me a curious thing to do. Does expertise just not matter any more? Must our possessions and preferences lie in the hands of others? What I see in this shift is the downgrading of rational capabilities. From subjective performers, we are turning into the objects of society. But why focus so much on Bentley et al.'s thoughts about the "herd-like" downshifting in the functional description of human behavioural processes (cf. sects. 1, 2.3, and 4)? I think psychologists should have greater wherewithal for interpreting this tendency and offering society the means for projecting itself intelligently.

The "out-sourcing" of mental activities is necessary in order to change types of activities, but physicians have been diagnosing similar things to the "decision fatigue" (sect. 2, para. 4) mentioned by Bentley et al.: Could myalgic encephalopathy or chronic fatigue syndrome be related to decision fatigue? These eventually are the related physiological implications that are cropping up in respect to a new set of identity factors of the individual in the west. As a matter of fact, the British National Health Services (NHS) "Choices" web page reports 250,000 syndrome cases of this type in the United Kingdom (National Health Services 2013); and 1,115 cases were reported within a population of approximately 144,000 (Olmsted County, Minnesota) through the half-acentury Mayo Clinic Rochester Epidemiology Project (cf. Kremers et al. 2011).

Since complex digital-shadow handling and the surcharge of opaque information is causing decision-taking to be difficult for the rational individual, won't strong individualism from the west wreak some havoc in the east? Having noticed now how handling identity in digital-shadow-rich populations has become a "nightmare" for many, one can conceptually picture people from the east becoming overly individualistic with respect to the standing canonical norms. I do not predict the language base in either east or west will be altered immediately. As the (western substratum-based) self endures increasing identifiability problems with respect to its former methods of going about this, the mind/ brain will either begin to fail pathologically or learn to appreciate its ability to employ relativism in new contexts.

\section{A map of where? Problems with the "transparency" dimension}

\section{doi:10.1017/S0140525X13001891}

\section{David Spurrett}

Department of Philosophy, University of KwaZulu-Natal, Durban, 4041, South Africa.

\section{Spurrett@ukzn.ac.za}

Abstract: Transparency is a fundamental concept in the target article by Bentley et al. But the text gives cryptic, inconclusive and sometimes conflicting suggestions as to what transparency consists of. Consequently, it is insufficiently clear what the north-south axis of the map of collective behaviour actually represents, or how to order behaviours by transparency.

The notion of transparency is among the few most fundamental concepts in Bentley et al.'s target article. Transparency, and its absence or opposite, define one of the two axes of the map of collective behaviour that is described and defended there. Unfortunately, it is far from clear what notion of transparency the authors have in mind.

The fact that Bentley et al. offer an equation in which transparency occurs (as $b_{t}$ in Equation 1, sect. 2) does not clarify what they 
mean by transparency. Rather, it highlights the challenge they need to meet. An equation represents purely formal relationships. To make an equation empirically significant the variables need interpretations. These should include some indication of what would count as determining a value for each variable. This is as true in physics as it is in the cognitive and behavioural sciences.

One reason it isn't clear how to understand statements about transparency in the target article, is that the term itself appears in some statements that purport to explain what it means. For example, in section 2 we are told that the north-south dimension of the map graphs the "transparency or opaqueness of the decision in terms of payoff" (sect. 2, para. 1). Shortly after, in the same paragraph, we read a "more formal" statement, according to which transparency concerns the "extent to which there is a transparent correspondence between an individual's decision and the consequences (costs and payoffs) of that decision." But, first, the more formal statement seems to say no more than the preceding one. Second, both contain the very term to be clarified.

We are also told that transparency is somehow related to payoffs. The article provides some encouragement for the view that transparency is a matter of knowing the payoff to be expected from a choice before it is made. We read, for example, that the north of the map "contains agents ... who know the impact their decisions will have on them" (sect. 2.1, para. 1; emphasis added), and that going south takes us to agents who are "less able to discern differences in potential payoffs" (sect. 2, para. 1; emphasis added). Unfortunately, we're also told that in the extreme north the impact of a decision is "immediately detectable" (sect. 2, para. 4; emphasis added), which suggests transparency has something to do with experienced consequences rather than ones known in advance. These possibilities are importantly distinct. The cognitive demands of reinforcement learning and those of predicting without experience are quite different. (They correspond to Dennett's [1996] distinction between "Skinnerian" and "Popperian" agents.)

The ambiguity between payoffs anticipated and experienced is mirrored in ambiguity over whether transparent payoff relations are simply known, or are the object of some kind of higherorder cognition. Sometimes the north is characterised in terms of what an agent can "discern" or "detect," suggesting that payoffs are simply known. (Although, to the south, apparently, agents might not know the "qualifications" of those they learn from, which suggests that knowledge of matters other than payoffs might also be part of transparency.) But we're also told that agents on the northern edge "clearly understand the rationale" for new behaviours, and engage in "conscious weighing of options" (sect. 2.2, para. 1). Again, these are importantly distinct: Agents could successfully learn which behaviours are rewarded (and by how much) without being capable of means-end rationality or in any other way having a rationale for their behaviour.

What is said about the opposite of transparency raises further confusing associations. So, at the extreme south (where $b_{t}=0$ ) we encounter "total indifference," suggesting that under conditions of full opacity agents have no preferences between options. In the deep south, "the probability of any particular choice among $N_{t}$ possible choices approaches zero (because $1 / N_{t}$ goes to zero as $N_{t}$ goes to infinity)" (sect. 2, para. 4; emphasis added). The claim here is that loss of transparency is an effect of the sheer number of options. But this seems confused. An agent could surely be entirely ignorant of the payoff difference between as few as two options (so minimal transparency occurs when $N_{t}$ is very small), and, conversely, know perfectly well that at least one option from a possibly infinite set pays handsomely.

On two occasions (in section 2) the notion of transparency is linked to a "landscape" of costs and payoffs. We are told that with northward movement, agents' decisions will be more "attuned" with this landscape, and that the landscape can be "represent[ed] by the function $U(\ldots)$ of costs and payoffs" (sect. 2, para. 4). But these references to landscapes dispel little of the mist surrounding the concept of transparency.
If $U(\ldots)$ refers to Eq. 1, then it isn't exclusively a function about payoffs, because that includes the (presumably comprehensive) "list of covariates" (sect. 2, para. 2) that "influence choice" at a time, as well as measures of popularity (of the choice) and influence (upon the individual agent of those already opting for the choice).

Perhaps the idea is that the net individual payoff portion of $U(\ldots)$ could be extracted separately for each available choice. That would enable us to define the height of the "landscape" for each option $(k)$. What we would have then would be a lookup table of payoffs and options, rather than a landscape. To organise the payoff-option pairings into a landscape, we would need to have criteria for relative proximity. (On a fitness landscape, for example, these could be provided by degrees of genetic similarity.) But what might provide homogenous criteria for degrees of proximity in the space of choices, so that the payoffs can be organised into a landscape? What topographic properties might the resultant landscape have? How are other components of $U(\ldots)$, such as relative popularity, to be represented on the landscape? The problem isn't that no answers to these questions are possible, it's that the target article gives no hint as to which we should have in mind.

The net result of all this is that the map defended in the target article belongs towards the (opaque) south of the very space it represents.

\section{Using big data to map the network organization of the brain}

\author{
doi:10.1017/S0140525X13001908
}

\begin{abstract}
James E. Swain, ${ }^{a, b, d}$ Chandra Sripada, ${ }^{a, c}$ and John D. Swain ${ }^{\mathrm{e}}$ ${ }^{a}$ Department of Psychiatry, University of Michigan School of Medicine, Ann Arbor, MI 48109; 'bepartment of Psychology, University of Michigan, Ann Arbor, MI 48105; 'Department of Philosophy, University of Michigan, Ann Arbor, MI 48105; ' $\mathrm{d} C h i l d$ Study Center, Yale University School of Medicine, New Haven, CT 06520; ${ }^{\mathrm{e} D e p a r t m e n t ~ o f ~ P h y s i c s, ~ N o r t h e a s t e r n ~ U n i v e r s i t y, ~}$ Boston, MA 02115.

jamesswa@med.umich.edursripada@umich.edu

John.swain@cern.ch

http://www2.med.umich.edu/psychiatry/psy/fac_query4.cfm?

link_name=jamesswa

http://www.Isa.umich.edu/philosophy/people/faculty/ci.

sripadachandra_ci.detail

http://www.physics.neu.edu/Department/Vtwo/faculty/swain.htm
\end{abstract}

Abstract: The past few years have shown a major rise in network analysis of "big data" sets in the social sciences, revealing non-obvious patterns of organization and dynamic principles. We speculate that the dependency dimension - individuality versus sociality - might offer important insights into the dynamics of neurons and neuronal ensembles. Connectomic neural analyses, informed by social network theory, may be helpful in understanding underlying fundamental principles of brain organization.

Neuroscientists are increasingly analyzing distributed patterns of connectivity between spatially dispersed brain regions, placing the topic of network interconnections at the center of the field (Behrens \& Sporns 2012; Bullmore \& Sporns 2012; Sporns 2012; Supekar \& Menon 2012). What lessons might Bentley et al.'s quadrant analysis of social networks have for understanding brain networks? We propose that the role of dependency - the west-east dimension of individuality versus sociality-might be particularly important for understanding the dynamics of neurons and neuronal ensembles.

Dependency is ubiquitous in graphs characterizing real-world phenomena, and it is useful to consider how it might arise. Analyses of random networks (also called random graphs) may begin with a collection of nodes joined by edges at random. A large body of results have been obtained: The subject goes back to the Erdös-Renyí model of 1959-1960 (see Erdös \& Renyí 1960), but 
the corresponding results bear little relation to graphs that arise in real-life situations, where edges are characteristically added with some dependence on edges that are already present.

A more interesting class of networks, which has received much recent interest, are so-called "small networks" in which most nodes are not directly connected to each other, but can be reached from every other by a small number of hops. This sort of network is often explained in the popular literature via the "Kevin Bacon Game" in which the nodes are actors and the edges connecting them represent their having appeared in the same movie. The question is then posed of how many edges one must follow (how many nodes one must hop between) to get from Kevin Bacon to any given actor. Each actor then gets a "Bacon number" which represents how many hops away from Kevin Bacon they are. Remarkably, most successful actors have a Bacon number less than or equal to 5. Such networks, in the context of the four-quadrant human decision-making map (Behrens \& Sporns 2012), would represent a trend toward the east-more sociality, and more chances to connect lead naturally to such networks. Such networks have many interesting properties including a tendency to have a small number of nodes with very high degrees of connectivity - something that can be looked for in (big) data. Similar patterns appear in anatomical connections in the brain (Sporns et al. 2002) and in synchronization networks of cortical neurons (Yu et al. 2008).

Various mechanisms can give rise to such networks, the most popular being the Watts-Strogatz (W-S) mechanism (Watts \& Strogatz 1998), which is constructed by iteratively rewiring a pre-existing graph (something which might be expected to model evolution on a pre-existing network), and the BarabásiAlbert (BA) model (Barabási \& Albert 1999), which is based on the notion of preferential attachment, where new nodes are added with connections made preferably to those which already are more connected. The BA model, for example, has been used to model the World Wide Web (www) where one might well expect that more people would add new links to a more popular (more linked to) site than to one less used. A general review can be found in Albert and Barabási (2002).

The BA model also gives rise to an interesting distinction from the W-S mechanism in that it gives rise to power-law or "scalefree" networks where the number of nodes with some number of connections depends as a power of that number. This gives rise to long-tailed distributions (Behrens \& Sporns 2012). Indeed, this behavior is found by Bentley et al. in their Figure 1 (target article, sect. 2). Again, this sort of behavior can be sought in big datasets and can give valuable information about the possible origin of a given network configuration. So far we have assumed a deviation from randomness (an appearance of structure) due to fairly deterministic processes where, even if edges appear randomly, their probabilities depend deterministically on other factors. Relatively little is known if one weakens this dependence by the addition of random noise.

As an aside, we note that the north-south axis is described as "the extent to which there is a transparent correspondence between an individual's decision and the consequence of that decision" (target article, sect. 2, para. 4). If we interpret this as a weakening of a direct cause-effect relationship, we suggest that this might indeed be modeled as noise-something that has been much less studied in the physics community, yet which could surely be added to models which have been considered, in some cases perhaps analytically, but also certainly via computer simulations. Spin-glasses are often used to model network dynamics (Binder \& Young 1986) where a temperature-like parameter represents noise, but this work tends to be done to represent correlations between activities at nodes rather than on the dynamics which drives the formation of edges - that is, the structure of the network itself.

Big-data approaches from the social sciences are already motivating significant new developments in characterizing brain networks. Over recent years, connectomic analyses of brain activity in large datasets have elucidated the network architecture of the brain (Behrens \& Sporns 2012; Sporns 2012; Supekar \& Menon 2012) and identified fundamental principles of the brain's graphical organization (Bullmore \& Sporns 2012). New approaches promise to shed light on brain networks implicated during specific cognitive tasks, such as altered network interrelationships during volition regulation of emotion (Sripada et al. 2013). Such brain mechanisms involved in specific cognitive tasks might ultimately be helpful in understanding brain-behavior responses to real and increasingly social stimuli-for example, parents responding to baby-cries (Swain \& Lorberbaum 2008; Swain et al. 2004; 2011), the complex array of social responses involved in parenting (Swain 2011) - and to broader societally directed behaviors such as altruism (Swain et al. 2012). Such approaches may be helpful in understanding underlying mechanisms of psychiatric disorders such as obsessive-compulsive disorder (Leckman et al. 2004; Mayes et al. 2005), generalized social anxiety, and autism that involve pervasively abnormal functioning in social domains.

\section{ACKNOWLEDGMENTS}

James E. Swain is partially supported by an Independent Investigator Award from the National Alliance for Research on Schizophrenia and Depression (Brain and Behavior Foundation), and the Michigan Institute for Clinical Health Research (UL1TR000433). Chandra Sripada is partially supported by NIH grant AA020297, Center for Computational Medicine Pilot Grant, and the John Templeton Foundation. John D. Swain is partially supported by the National Science Foundation.

\section{Mapping collective emotions to make sense of collective behavior}

\author{
doi:10.1017/S0140525X1300191X
}

\section{Maxime Taquet, ${ }^{\mathrm{a}}$ Jordi Quoidbach, ${ }^{\mathrm{b}}$ Yves-Alexandre de Montjoye, ${ }^{\mathrm{c}}$ and Martin Desseilles ${ }^{\mathrm{d}}$}

aCTEAM Institute, Catholic University of Louvain, B-1348 Louvain-la-Neuve, Belgium; 'bepartment of Economics and Business, University Pompeu Fabra, 08005 Barcelona, Spain; ' Massachusetts Institute of Technology, The Media Laboratory, Cambridge, MA 02139-4307; 'Department of Psychology, University of Namur Medical School, B-5000 Namur, Belgium. maxime.taquet@uclouvain.be jordi.quoidbach@upf.edu yva@mit.edu martin.desseilles@unamur.be

http://maximetaquet.com/

http://www.quoidbach.org/

http://www.demontjoye.com/

http://mentalhealthsciences.com/

Abstract: While Bentley et al.'s model is very appealing, in this commentary we argue that researchers interested in big data and collective behavior, including the way humans make decisions, must account for the emotional factor. We investigate how daily choice of activities is influenced by emotions. Results indicate that mood significantly predicts people's decisions about what to do next, stressing the importance of emotional state on decision-making.

Bentley et al. propose that decision-making can be understood along two dimensions. The first dimension represents the degree to which an agent makes a decision independently versus one that is socially influenced. The second dimension represents the degree of transparency in the payoffs and risks associated with the decisions agents make. While Bentley et al.'s model is very appealing, we argue that emotions, a key element to understand the way humans make decisions, are missing.

From early theorizing by William James, to Antonio Damasio's work on somatic markers, decades of research consistently have shown that emotions play a central role in the decision-making process (see, e.g., Bechara \& Damasio 2005; Loewenstein 2000). For instance, in economic decisions, fear leads to riskaverse choices, whereas anger leads to risk-seeking choices 

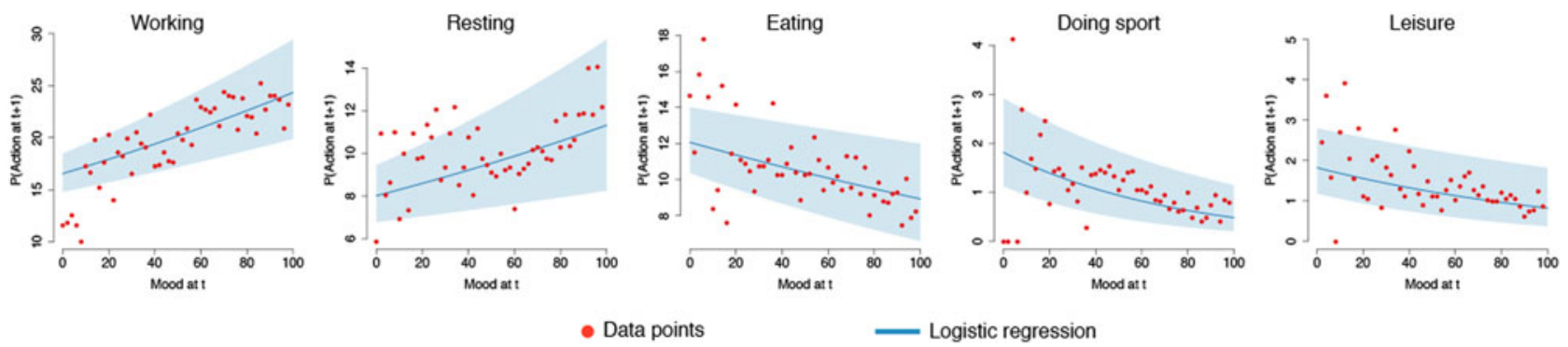

Figure 1 (Taquet et al.). Five activities are significantly predicted by mood. The figure presents the data in red (aggregated by mood in bins of 2 levels: $0-1,2-3, \ldots, 99-100)$ and the corresponding logistic curve in blue, corrected for mood at time $t+1$ and the interaction between mood at time $t$ and time between tests. The shaded area corresponds to two standard errors above and two standard errors below the curve. A color version of this image is available at http://dx.doi.org/10.1017/S0140525X1300191X.

(Lerner \& Keltner 2001). In medical decisions, positive affect improves physicians' clinical reasoning and diagnosis (Estrada et al. 1997). In ethical decisions, social emotions such as guilt can lead individuals to choose ethically (Steenhaut \& Van Kenhove 2006). These studies, among many others, strongly demonstrate that emotions shape most of our decisions. Researchers in microeconomics, health, or ethics are already taking emotions into account. It is now time for big-data and collective behavior researchers to recognize the importance of the emotional factor in the decision-making process.

In this commentary, we illustrate the importance of emotions to predict people's behavior using the example of a big dataset derived from an ongoing large-scale smartphone-based, experience-sampling project (available at: http://58sec.fr/). Specifically, we show that the happiness that individuals experience at time $t$ reliably predicts the type of activities they choose to engage in at time $t+1$.

Subjects voluntarily enroll in the experiment by downloading and installing the mobile application " $58 \mathrm{sec}$ ". They are then presented with questionnaires at random times throughout the day-henceforth referred to as tests. Random sampling is ensured through a notification system that does not require users to be connected to the Internet. The minimum time between two tests is set to one hour to avoid large artifactual auto-correlations between answers to the same question in consecutive tests. At each test, participants are asked to rate their current mood on a scale from 0 (very unhappy) to 100 (very happy) and to report which activity they are currently engaged in, among other questions. Activities can be selected from a list of 25 non-mutually exclusive choices that, among other activities, include doing sports, working, resting, praying/meditating, shopping, and commuting.

To illustrate the dynamic between emotion and decisionmaking, we randomly selected 5,000 people from our database and investigated how their daily choice of activities (e.g., whether one decides to spend the evening working out or watching TV) is influenced by their emotion. Specifically, we tested how much mood reported within one test (time $t$ ) predicts the activity reported within the next test (time $t+1$ ). For each possible activity, a logistic regression model is fitted for the probability of the activity (dependent variable) as a function of previously reported mood (independent variable). Mood at time $t$ may be correlated to mood at time $t+1$, which itself correlates with the activity at time $t+1$. To cross out this indirect effect of emotion on decision, mood at time $t+1$ is included in the model as a covariate. Emotions closer in time to a decision may better predict its outcome. To capture this notion, we included an interaction term between the (random) time between the two tests and mood at time $t$.

Big datasets allow many variables to be compared simultaneously without diluting the effect of interest in the correction required to account for the multiple comparisons. For the same underlying effect size, the $p$-value will indeed decrease as the

number of data points increase. More data points therefore reduce the number of Type II errors (false negatives), for a constant Type I error rate (false findings). Accordingly, the threshold on the $p$-value can be reduced from its typical value (e.g., 0.05 ) to also decrease the number of findings that are false. In this study, we set the significance threshold at $p<0.001$ to increase the confidence in our findings.

Significance testing was carried out on the coefficient (Beta ${ }_{\text {pred }}$ ) of mood at time $t$ in the prediction of each action at time $t+1$. The resulting $25 p$-values were corrected for multiple comparisons using Bonferroni correction. Each of the 5,000 subjects participated in an average of 13.1 tests. Those subjects who participated in only one test were discarded since their test results did not convey information about the prediction of emotion on decision. This gave rise to a total of 59,663 data points from which the logistic regression could be fitted.

Five activities were significantly predicted by $\operatorname{mood}$ at the $p=$ 0.001 threshold after Bonferroni correction (Fig. 1): working $\left(\right.$ Beta $\left._{\text {pred }}=0.48, p<10^{-12}\right)$, resting $\left(\right.$ Beta $\left._{\text {pred }}=0.38, p<2 \times 10^{-4}\right)$, eating $\left(\right.$ Beta $\left._{\text {pred }}=-0.34, p<5 \times 10^{-4}\right)$, doing sports $\left(\right.$ Beta $_{\text {pred }}=$ $\left.-1.3, p<10^{-9}\right)$, and leisure $\left(\right.$ Beta $\left._{\text {pred }}=-0.81, p<3 \times 10^{-4}\right)$. These results indicate that mood significantly predicts people's decisions about what to do next, stressing the importance of emotion on decision-making.

Big data and large-scale experience sampling through pervasive technologies offer unprecedented opportunities to understand collective behaviors. Such methods are particularly suited to study collective behavior as its causes often involve complex interactions between sensitive variables. One archetypal example of such collective behavior is decision-making which involves independence of the agent, transparency of the payoffs, and emotional state.

\section{Conformity under uncertainty: Reliance on gender stereotypes in online hiring decisions}

doi:10.1017/S0140525X13001921

\section{Eric Luis Uhlmann ${ }^{\mathrm{a}}$ and Raphael Silberzahn ${ }^{\mathrm{b}}$ \\ aManagement and Human Resources Department, HEC Paris - School of Management, 78351 Jouy-en-Josas, France; ' Judge Business School, University of Cambridge, Cambridge CB1 2AG, United Kingdom. eric.luis.uhlmann@gmail.com \\ http://www.socialjudgments.com/ \\ rts27@cam.ac.uk \\ http://www.jbs.cam.ac.uk/programmes/research-mphils-phd/phd/phd- students-a-z/raphael-silberzahn/}

Abstract: We apply Bentley et al.'s theoretical framework to better understand gender discrimination in online labor markets. Although such settings are designed to encourage employer behavior in the 
northwest corner of Homo economicus, actual online hiring decisions tend to drift southeast into a "confirmation bias plus weak feedback loops" pattern of discrimination based on inaccurate social stereotypes.

Bentley et al. provide a framework for understanding online behavior which predicts that in online settings people often do not independently and logically evaluate the available information, but instead conform to common cultural biases in their judgments. In the present commentary, we apply the authors' framework to better understand gender discrimination in online labor markets (Bonet et al. 2013; Cappelli \& Keller, in press).

More so than traditional labor markets, online markets for temporary labor are meant to be closer to the economic ideal of a perfect market, with standardized presentation of candidates' profiles and high transparency of worker characteristics important to the job such as scores on standardized tests of relevant skills, employment history, and job interests. This richness of information allows employers to assign jobs to workers with appropriate skills at market value; and for workers, such transparency should facilitate access to jobs based on merit with hiring decisions based solely on considerations of value for money (Horton 2012; although see Bidwell et al. 2013). Hence, online labor markets are designed to encourage employer behavior in what Bentley et al. refer to as the northwest corner of Homo economicus: independent decisions based on a rich source of transparent information.

However, our findings indicate that, consistent with the theorizing of Bentley et al., actual online hiring decisions tend to drift southeast, into what the authors refer to as a "confirmation bias plus weak feedback loops" pattern (target article, sect. 2.3, para. 3 ). The desire to reduce the amount of information processing required to evaluate numerous choice options along multifold criteria can lead individuals to conform to social conventions and heuristics (Christensen-Szalanski 1978; 1980; Payne 1982; Payne et al. 1988; 1993; Timmermans 1993; Wright 1975). One very commonly used type of heuristic is that of cultural stereotypes (Bodenhausen 1988; 1990). In an online hiring market, applicants for each job are numerous, and attracting more than one hundred applicants in the first hour after posting the job is not uncommon. Although detailed and relevant information for all applicants is available, employers may not make full use of such information. Faced with the overload of information associated with the number of applicants, employers appear to rely heavily on cultural stereotypes about men and women to reduce uncertainty and simplify their decisions.

We find that controlling for the actual number of men and women interested in the job category, female workers are less likely than chance to be hired for stereotypically male jobs such as programming, and more likely than chance to be selected for stereotypically female jobs such as customer service (Silberzahn et al., under review). Because of such discrimination in selection, the discrepancy between workers' aspirations in terms of pay and job status and the jobs they actually obtain is much larger for female than for male workers. Thus, in online labor markets we find the same pattern of stereotype-based discrimination observed in other settings (Eagly \& Karau 2002).

We also find evidence of a much more subtle form of gender discrimination that emerges in temporary online labor markets. Past research indicates that social stereotypes foster uncertainty about the capabilities of counter-stereotypical individuals (Bodenhausen 1988; Darley \& Gross 1983; Eagly \& Karau 2002; Heilman 2001; Perry et al. 1994). Based on this research, we theorized that stereotype-based uncertainty would be reflected in the kind of contract offered. Specifically, we hypothesized that temporary workers hired for jobs not stereotypically suited for their gender would be offered contracts that allow the employer to more easily terminate the relationship in case of a poor performance (Silberzahn et al., under review). Consistent with this hypothesis, women actually hired for stereotypically male jobs are more often paid by the hour rather than offered fixed contracts that span the entire length of the project, a risk-averse practice that suggests an uncertainty about their abilities on the part of employers. In contrast, for stereotypically female-typed jobs, female workers are comparatively more likely to receive fixed contracts. Supporting the idea that these effects are driven by uncertainty about the performance of counter-stereotypical individuals, the opposite pattern emerges for male workers, who are more likely to be paid by the hour for traditionally female jobs. Discrimination in the type of contract offered leaves workers in jobs not typical for their gender in more vulnerable and precarious employment arrangements from which they can easily be fired.

A confirmation bias appears to play a role in discrimination in selection and contract type, as employers conform to gender stereotypes in their decision making even though such stereotypes are wholly inaccurate. In fact, our data indicate that female workers interested in stereotypically male jobs have, on average, higher levels of skills in the relevant domain than their male counterparts. Further, temporary online labor markets may be characterized by what Bentley et al. describe as a weak feedback loop, in which expectations biased by social stereotypes rarely have a chance to be disconfirmed. The typical pattern that emerges is that an employer chooses men and women for jobs stereotypical of their gender, is satisfied with the workers' job performance, and proceeds to make further stereotypical hires. In contrast, it is far less common for employers to hire women for traditionally male work under a long-term contract, which drastically reduces their opportunities to unlearn their stereotypes through experience.

These findings provide empirical support for Bentley et al.'s argument that rather than necessarily encouraging rational information use and utility maximization, online contexts are highly prone to human failings such as conformity to common social beliefs and confirmation bias. Employers systematically and repeatedly violate principles of economic rationality by not hiring highly qualified female workers. Moreover, rather than maximizing their job preferences and outcomes in online labor markets, female workers appear to be systematically underemployed relative to both their skills and job interests. Technological innovations such as online labor markets provide employers and workers worldwide with opportunities for efficient matching of skills and work. However, the advantages of online technology are not sufficient to overcome deeply entrenched biases in human decision making.

\section{Interaction between social influence and payoff transparency}

doi:10.1017/S0140525X13002501

\section{Xinyue Zhou, ${ }^{a}$ Wenwen Xie, ${ }^{a}$ and Maolin $\mathrm{Ye}^{\mathrm{b}}$ \\ ${ }^{a}$ Department of Psychology, Sun Yat-sen University, Guangzhou, 510275, China; ${ }^{b}$ School of Management, Jinan University, Guangzhou, 510632, China. zhouxyue@mail.sysu.edu.cn xiewenw@mail2.sysu.edu.cn maolinye@163.com \\ http://www.psy.sysu.edu.cn/teacher/tea01/tea0119/index.htm http://pro.jnuedu.cn/T_ShowDetails.aspx?T_ID $=87$}

Abstract: Social influence and payoff transparency interact with each other to influence decision making. Social influence masks payoff transparency, and lacking transparency drives people to seek social influence. Moreover, our survey supports our claim by showing that social influence and payoff transparency correlate with each other $(r(53)=-.71)$. Bentley et al.'s model can be revised to accommodate the covariance.

In the target article, Bentley et al. have created a multiscale "map" in an attempt to capture the essence of decision making along two axes: social influence and transparency of payoffs. Grounded in discrete-choice approaches and with a few simplifying assumptions (sect. 2, para. 7), this concept tool sheds light on understanding 
Response/Bentley et al.: Mapping collective behavior

different domains of human decision making. In what follows, we argue that this map fails to capture the relationship between social influence and payoff transparency.

Bentley et al. have interpreted their model as a "principal-components representation" (target article Abstract), which assumes that two axes are orthogonal to each other. However, it is well documented in social psychological literature that social influence and payoff transparency influence each other to a great extent. When two parameters are correlated with each other, one axis should not be perpendicular to the other axis (DeVellis 2012, p. 142). Thus, the map as well as the formula at the start of section 2 should be revised to accommodate the hypothesized angle that represents the covariance of the two key parameters. Even though Bentley et al. did acknowledge this interaction in some places (e.g., "Intuitively, what is happening here is a pileup of correlated behaviors caused by the interaction of social influences coupled with strong enough intensity of choice"; target article, sect. 2.2.1, para. 2), when they compare their map to a Google map (sect. 1, para. 6), they run the risk of misleading the readers into believing that the two axes are perpendicular to each other.

Decision-making is a dynamic process in which transparency and social influence invariably interact with each other. Under social influence, even the most transparent decision-making task becomes ambiguous. For example, in a classic conformity experiment (Asch 1951), participants were instructed to complete a perceptual task in which they had to match the length of a given line with one of three comparison lines. Although the correct judgments were easy to make, $75 \%$ of the participants made an incorrect judgment in at least one trial when all the confederates gave the same wrong answer. Thus, social influence can mask even the most transparent decision. Under social influence, even simple tasks like line comparisons are not as transparent as when there is no social influence (Asch 1951; 1952; 1956). Moreover, other studies have shown that social influence could change one's cost-benefit estimation of a decision (Louis et al. 2005).

On the other hand, people are more likely to seek social influence when facing opaque decisions than when facing transparent decisions (Deutsch \& Gerard 1955; Stasser \& Davis 1981). Deutsch and Gerard (1955) also asked participants to compare lines just like Ash did in the aforementioned study. In half of the trials (visual condition), the lines were physically present when participants and confederates made their judgments. Thus, in this condition, the decisions are fairly transparent. However, in the other condition, the lines disappeared before the participants had the opportunity to make judgments; hence, the decisions depended on memories and were less transparent than decisions in the first condition. Results showed that participants in the second condition were more likely to conform to social influence than those in the first condition.

When people have little knowledge about what to base their decisions on, it is helpful to imitate the successful judgments or to average the judgments of others to exploit the "wisdom of crowds" (Gigerenzer \& Gaissmaier 2011). Findings from neural imaging researches support this claim by showing that social information processing and decision making have shared neural substrates (Tomlin et al. 2013). Opaque decisions drive people to seek social influence.

We recently conducted a survey (Zhou 2013) to examine whether transparency and social influence correlate with each other in daily decision making. We asked 55 participants to think about one upcoming decision they have to make in real life. Participants wrote down keywords best describing this decision. Next, participants rated how transparent the decision payoff is to them and how much social influence they are under in making the decision on an 11-point scale $(0=$ not at all; $10=$ very much). The order of these two questions was counterbalanced so that half of the participants rated the transparency first and half of them rated the social influence first. Regardless of which question was asked first, social influence and payoff transparency turned out to be negatively correlated, $(r(53)=$ $-.71, p<.0005)$. These results support our argument that social influence and transparency interact with each other to a great degree and cannot be considered as independent dimensions.

In conclusion, we agree that Bentley et al. have provided a comprehensive map to evaluate collective behaviors in the big-data era. We also agree that this map will lead to new promising hypotheses on human decision making. However, Bentley et al. have not represented the interaction of the two axes on their map in a precise manner that would reflect the factual nature of the interaction. The accurate depiction of the covariance of social influence and payoff transparency is critical because it exerts direct impact on decision making in the digital age. The map proposed in the target article would function more quantitatively and accurately if it were revised taking the interaction between the two dimensions into account.

\section{ACKNOWLEDGMENTS}

The authors wish to thank Boya Yu and Kornel Tomczyk for their helpful comments on an earlier draft of this commentary. This work was supported by the Key Program and General Program of National Natural Science Foundation of China (Grants 91124004 and 31171002) and Distinguished Young Scholars Program (No. 13wkpy87).

\section{Authors' Response}

\section{More on maps, terrains, and behaviors}

doi:10.1017/S0140525X1300277X

\section{R. Alexander Bentley, ${ }^{a}$ Michael J. O'Brien, ${ }^{\mathrm{b}}$ and William A. Brock ${ }^{\mathrm{c}, \mathrm{d}}$}

${ }^{a}$ Department of Archaeology and Anthropology, University of Bristol, Bristol BS8 1UU, United Kingdom; ' ${ }^{\mathrm{b}}$ Department of Anthropology, University of Missouri, Columbia, MO 65211; ' Department of Economics, University of Missouri, Columbia, MO 65211; 'Department of Economics, University of Wisconsin, Madison, WI 53706.

r.a.bentley@bristol.ac.uk obrienm@ missouri.edu
wbrock@scc.wisc.edu
http:// www.alex-bentley.com
http://cladistics.coas.missouri.edu
http://www.ssc.wisc.edu/ wbrock/

Abstract: In a recent New York Times column (April 15, 2013), David Brooks discussed how the big-data agenda lacks a coherent framework of social theory-a deficiency that the Bentley, O'Brien, and Brock (henceforth BOB) model was meant to overcome. Or, stated less pretentiously, the model was meant as a first step in that direction - a map that hopefully would serve as a minimal, practical, and accessible framework that behavioral scientists could use to analyze big data. Rather than treating big data as a record of, and also a predictor of, where and when certain behaviors might take place, the $\mathrm{BOB}$ model is interested in what big data reveal about how decisions are being made, how collective behavior evolves from daily to decadal time scales, and how this varies across communities.

\section{R1. Introduction}

We are encouraged and inspired by the rich variety of commentaries, noting that, in general, commentators found something useful in our map of decision making. MacCoun, for example, points out his similar model for 
binary decisions; Durlauf appreciates its elegance in simplicity; Ross discusses how economics has already explored the four quadrants of our map; and Uhlmann \& Silberzahn apply the map quadrants to gender bias in a bigdata study of employment search patterns. A good number of commentators even propose their own adaptation of the map quadrants, often as an added dimension, including emotion (Buck; García, Torralba, \& González [García et al.]; Ruths \& Shultz; Taquet, Quoidbach, de Montjoye, \& Desseilles [Taquet et al.]), network structure (Fortunato, Saramäki, \& Onnela [Fortunato et al.]; Swain, Sripada, \& Swain [Swain et al.]), sociallearning biases (Le Mens; Mesoudi; Pfister \& Böhm), and cultural conception of time (Norgate, Davies, Speed, Cherrett, \& Dickinson [Norgate et al.]). Others sought refinement of either the east-west dimension (O'Donnell, Falk, \& Konrath [O'Donnell et al.]; Le Mens) or the north-south dimension (Spurrett). Several contributed very useful ways forward in mapping the terrain of the map in considerably more detail (Analytis, Moussaïd, Artinger, Kämmer, \& Gigerenzer [Analytis et al.]; Christen \& Brugger; Hopfensitz, Lorini, \& Moisan [Hopfensitz et al.]; Keane \& Gerow; Le Mens; McCain \& Hamilton; Moat, Preis, Olivola, Liu, \& Chater [Moat et al.]; Reader \& Leris). Objections to our map include oversimplification (Godzińska \& Wróbel; Reader \& Leris) and misrepresentation of the orthogonality of its axes (Bookstein; O'Donnell et al.; Zhou, Xie, \& Ye [Zhou et al.]).

Our response has three parts: the map, its terrain, and some speculation about mapping the effect of big data on collective behavior, including the kinds of self-aware "looping" effects discussed by Christen \& Brugger; Fan \& Suchow; O'Donnell et al.; Roesch, Stahl, \& Gaber (Roesch et al.); and Schmidt.

\section{R2. The map}

The theory used as the foundation for the BOB map - discrete choice - is extremely broad (Ben-Akiva et al. 2012), and the map is a tool to help behavioral scientists navigate the large research terrain of its many applications. The map is intended to facilitate interdisciplinary communication and insights across different phenomena and different scales of analysis, from big-data statistics to qualitative observation at the individual scale. As Ruths \& Shultz put it, the problem is highly dimensional, and the BOB map provides a framework on which future work can productively build. Spurrett takes us to task for fuzzy definitions of transparency and payoffs, but we think this flexibility is necessary to get people to use it as a basis for interdisciplinary communication and big-picture research, along the way making their own adaptations and modifications. To communicate with a business audience or public policymakers, for example, the north-south axis might be presented as extending from few choices in the north to many overwhelming choices in the south. Alternatively, as Buck advocates, the northsouth axis might instead extend from "rational" to "emotional," whereas in discrete-choice economics it would be called the "intensity of choice." As we specify below, it all corresponds to closely related formulations mathematically.

These different shades of interpretation may provide multiple proxies to be measured in big data, which
Godzińska \& Wróbel rightly call for. For it the map to be applicable to decision making, anywhere from prehistory to the era of big data, we need a minimalist structure that allows for added elements such as emotions collected through surveys or text mining, different concepts of time, kinship or other cultural constructions recorded in anthropology, or the millennia of material culture in the archaeological record. Consider the vast range of scales from the population-level patterns in big-data studies of language use in online social media to the activity within individual brains. The spirit of our map is to use big data to generate hypotheses that are then tested by other means (including qualitative) at the individual/group level, which Godzińska \& Wróbel invite us to consider, or even the level of neurobiology discussed by O'Donnell et al. We agree with Le Mens and with Roesch et al. that future uses of big data will include more sensitive measurements and more varied sources of information as the braincomputer interface develops. The BOB map provides a means of bridging scales of time and population. As Keane \& Gerow and Roesch et al. point out, big-data literature puts a microscope on individuals but often underplays the complex distributional effects and dynamics that are visible at this large scale but also require a time depth.

Ross misunderstood us when he stated that we were relegating all economics to the northwest quadrant; we were putting only Homo economicus there. We actually see most of behavioral economics as lying just east of the northwest quadrant. Nevertheless, while we were careful not to map whole disciplines or individual researchers into the quadrants, we recognize that the bulk of literature in any one (sub)discipline tends to gravitate toward one of the quadrants.

Godzińska \& Wróbel also see the map as too simplified to capture the essence of decision making, but most commentators accepted our invitation to fill in the map. The engagement of responders and the diversity of proposals to adapt the map help justify its simplicity, as Durlauf and MacCoun discuss. Even if a more complicated shape, such as the globe or tetrahedron suggested by Bookstein, could better capture certain interrelations, the cost would be the interdisciplinary common ground, and few in the $B B S$ community would adopt these more complicated heuristic models. Furthermore, a more complicated geometry might make things worse by assuming too much. In other words, Bookstein rightly reminds us that "the map is not the territory." We agree; the simple geometry is deliberate so that few would make this mistake. The simple two-dimensional form invites others to apply it or even modify it to suit their interests.

Together with Bookstein, Zhou et al. and Godzińska \& Wróbel object to the "structuralist" dichotomy we have implied by the orthogonal axes, maintaining that transparency itself is socially influenced. Reader \& Leris usefully caution against treating payoff transparency as a single entity, reminding us that transparency and the costs of decision making likely determine whether social or individual information is used. Zhou et al. present survey results in which social influence correlates with payoff transparency. We do not dispute either that $b_{t}$ and $J_{t}$ may correlate, or that in discrete-choice theory covariates that go into determining social influence and payoff transparency may indeed be correlated. We doubt, however, that the correlation will be the same in all cases, and one 
can easily envision situations where the two are anti-correlated or uncorrelated. Rather than assume some fixed correlation, the map is intended to represent how these shifts in decision making happen in any possible direction rather than just on fixed diagonals of assumed covariance.

\section{R2.1. Customizing the map}

So far, we have explored the map as we presented it, but as we noted earlier, we welcome the proposals to customize the map with added dimensions. Norgate et al. suggest that a crucial added dimension would represent the continuum between perception of "clock time" versus "event time," noting that the big-data era may be shifting societies from clock time back to event time, which presumably is at the primordial end of the spectrum. This relates the transition of our digital era to the classic anthropological formulation of a prehistoric transition from immediate return (hunter-gatherer) to "delayed return" (e.g., agricultural) societies. More generally, it fits very well into the discussion of forward-looking agents, which we discuss in the following section. Norgate et al. point out the existence of cultures with an identifiable time orientation toward the future, which Moat et al. have shown in their big-data studies (e.g., Preis et al. 2012; 2013) to have economic advantages.

Several commentators emphasize the relevance of emotions in decision making. We confess we had considered emotions to be a proximate cause of a decision, but the arguments of Buck, García et al., Ruths \& Shultz, and Taquet et al. are compelling concerning the fundamental importance of emotions to decision making. There are several ways of introducing emotions. One is to integrate them as a third dimension to the map, as proposed by García et al. Alternatively, emotion could be treated as another covariate in dynamic extensions of discrete-choice theory, which can accommodate such forms of decision making (Ben-Akiva et al. 2012). Perhaps most intriguing is the suggestion by Buck to modify the northsouth dimension in order to reflect emotions directly, so that the continuum ranges from purely emotional decision making (rather than opaque) in the south to purely rational (rather than transparent) in the north. In fact, if emotions can be used as a proxy for transparency or intensity of choice, then this presents a complementary means of measuring latitude on the map. This could be calibrated through various big-data measures of emotions, such as the smartphone self-assessments that Taquet et al. discuss (and which they correlate with decisions), or the frequencies of word stems on large sources of language use such as Twitter or Google's Ngram viewer (Acerbi et al. 2013; Lampos \& Cristianini 2012; Tausczik \& Pennebaker 2010).

We attempted to relate the map to generalized network structures in our Figure 8, and Fortunato et al. have proposed adding network structure as a third dimension, from highly clustered to fully connected networks - a dimension that is central to the famous small-world network formulation of Watts and Strogatz (1998). Swain et al. speculate that the east-west dimension might offer important insights into the dynamics of neural connectivity in smallworld properties of brain networks implicated during emotion, social stimuli, social anxiety, and autism. Because small-world networks have been investigated, both within the brain and between brains, network theory provides another unifying framework, as indeed networks have much to say about the short-tailed versus long-tailed distributions we discuss in reference to the map. Fortunato et al. point out that the collective outcome depends on social-network structure the more that agents stick to their choice rather than perpetually updating. This updating is nearly equivalent to adding noise and moving southward on the map, and as we suggested in Figure 8, specific network structure is probably more important in the northeast than in the southeast.

\section{R3. The terrain}

Several commentators contributed useful ways forward in mapping the terrain of the map in more detail. This could be through measurement-such as sophisticated data extraction (Moat et al.) or subtle changes in distributions (Keane \& Gerow)-or through multistage decision models (Analytis et al.; Hopfensitz et al.; McCain \& Hamilton), or through the remarkably simple addition of "contour lines" (Christen \& Brugger). We see the computer simulation by Analytis et al. as consistent with the predictions of our map: We just need to reverse the columns of their figure such that their "popularity-cue heuristic" model and its associated uniform distributions is in the west and their "popularityset heuristic" model is in the east. In their two-stage decision model, each agent first eliminates the majority (e.g., 90\%) of options. Agents following the popularity-set heuristic then choose among the shortlisted items through social influence, with probability proportional to popularity. This leads to just the kind of right-skewed distributions that we would expect in the east. Because agents choose the best from among only a 10\% random sample, rather than from among all samples, the popularity-cue heuristic yields a more uniform distribution, consistent with the noisy southwest. In other words, the shortlisting stage of the popularity-cue heuristic is random selection, that is, pure southwest $(b=0)$.

Similarly, any social influence under the popularity-cue heuristic is fairly weak because it is activated only after shortlisting and only as a "fourth attribute" among three other attributes that remain reflective of quality. This repeated $10 \%$ random-sampling process weakens the tracking of quality, and as a result, the popularity of choices increases slowly in the direction toward the item of highest quality (from 0 to 100). Referring to the concern of Roesch et al. about rate of change, the popularity cue may only gradually sort out the best choices from the worst. Perhaps after more time steps, the gradient would be steeper from the worst to best (item 1 to item 100). We might therefore categorize the popularity-cue heuristic as being in the southwest quadrant as a result of the random sampling in step one, but in the northeast quarter of that quadrant because of the transparency $(b>0)$ and weak social influence in step two.

Like Analytis et al., who consider a two-stage process, Hopfensitz et al. also study games that have multiple stages. These games may be usefully studied in the extended BOB framework of our response here by modeling them as nested Logit Quantal response games where the first nest is the set of games one chooses to play in the first period, and the second nest is the set of strategies 
of the game chosen in the first period. The analog of $(b, J)$ would be $(b, J)$ for the choice of game in the first period and $\left(b^{\prime}, J^{\prime}\right)$ for the second-period strategy choice of the game played in the second period. The choice of game in the first period includes the "game" of an "outside option" as in the commentary by Hopfensitz et al. Future research in multistage games proposed by Hopfensitz et al. is promising.

Similarly, McCain \& Hamilton convince us that including social interactions and quantal responses at each stage is an exciting agenda for future research. McCain \& Hamilton raise the interesting question of what happens if $(b, J)$ vary across quadrants of the map in the context of anti-coordination games (Bramoullé 2007). Imagine there is a stoplight that has a large but finite penalty if you run a red light, and imagine their Drive On game being played by a pair of players with foggy glasses (a small $b$ ). When $b=0$, each player plays "go" with probability $1 / 2$ and plays "wait" with probability $1 / 2$, and the probability of a crash is $1 / 4$. But when $b$ is infinite, the player facing a red light waits and traffic proceeds ideally.

We have emphasized that the $J_{t}$ parameter is an attempt to capture social influences, especially those types of influence that possess a "social multiplier" of policy relevance, which was stressed by Manski (1993) as being different from "spurious social effects" (Shalizi \& Thomas 2010). Like Mesoudi and Le Mens, Ruths \& Shultz are unsatisfied with the way we lump together all social-learning biases into the east. The map does, however, roughly cover the intensity of social tie tracked along the north-south dimension in the east. This makes it relevant to Granovetter's (1973) strong versus weak social ties and how the strength of these ties correlates with emotional closeness (e.g., Hill \& Dunbar 2003) - issues to which O'Donnell et al. refer. Hence, the north-south dimension does distinguish between the adaptive ratchet in the northeast, where social learning adaptively focuses on the most useful role models, and unbiased imitation in the southeast.

The Arab Spring, mentioned by Roesch et al. and O'Donnell et al. may be an example of the ambiguity of the effect of social media. When Gladwell (2010) pointed out "why the revolution will not be tweeted" - coincidentally just weeks before the Arab Spring uprising-he meant that revolutions require the strong social influence of the northeast, such as face-to-face interaction, rather than the weak ties of social media in the southeast. Social media are good for retrieving stolen cell phones left in cabs but not for carrying out revolutions, the success of which relies on organized hierarchy, not on here-todaygone-tomorrow social networks.

We are very aware that, as Ruths \& Shultz point out, sigmoidal adoption curves may indicate social learning but that other models of independent learning (the simplest assuming a normal distribution of independent response times) can produce the same result (e.g., Bentley et al. 2012; Brock \& Durlauf 2010; Hoppitt et al. 2010; Shultz 2003). This is why we propose distributions as a primary pattern for estimating $J$ along the east-west axis of social influence. This tool can be refined, and we agree completely with Keane \& Gerow that exploring the dynamics of how diagnostic popularity distributions change through time would refine the geography of our map within each quadrant. Fortunato et al. describe their insight from the precise popularity distribution of
Wikipedia pages, and Keane \& Gerow provide a nice example of how Zipf's Law in verb-phrase popularity became more "winner take all" among financial-media coverage as the stock-market crisis unfolded. Roesch et al. suggest that we could use "the velocity of change of decisions" and plot "vectors" on the map to show which way things are moving (vector direction) and at what rate (vector magnitude).

Although distributions are used to characterize the eastwest axis, we agree with Analytis et al. that distributions may not be particularly diagnostic of transparency along the north-south axis. This is determined by the parameter $b$, which is sometimes called the "intensity of choice." It measures the level of noisiness in choice-for example, when $b=0$, noise in choice is so large that choice is completely random over the choice set. When $b=\infty$, transparency in the relative values of the payoffs to each choice is so high that there is no doubt whatsoever which choice yields the highest payoff. The intensity of choice, $b_{t}$, is a precise and useful way to model the concept of "transparency" at each date $t$, where (1) $b_{t}=0$ corresponds to the lowest level of transparency and the farthest south on our map, and (2) $b_{t}=\infty$ corresponds to the highest level of transparency and the farthest north on our map. We realize that this modeling and conceptualization of "transparency" will not capture all useful interpretations, but we believe that it does capture a useful subset and makes a useful linkage to the very large and successful discrete-choice literature (Ben-Akiva et al. 2012).

The north-south axis (the $b_{t}$ axis) is a useful way of looking at the gain in precision of predicting the future using big-data sets. Prediction requires an expectation by forward-looking agents, and this applies to social transparency, as we discuss below regarding the fascinating future of "looping," to use the term of Christen \& Brugger. By linking our approach to some classes of social interactions games, for instance, Hofensitz et al. suggest that the impact of social ties will be different in the north than in the south. We use their suggestions as an opportunity to explain the theory behind our Equation 1 of $\mathrm{BOB}$, which lies in the domain of logistic quantal response games and quantal response (Nash) equilibria (McKelvey \& Palfrey 1995) and has been extended to include social interactions and covariates (Blume et al. 2011; Brock \& Durlauf 2001a; 2006). Let there be $G$ groups with $I$ players in each group. We can think of $I$ as being a large number so that the law of large numbers gives a good approximation in what follows. Assume the groups are disjoint, that is, non-overlapping, for simplicity. As a stochastic best-reply function for player $i$ at date $t$, consider the following modification of BOB's Equation 1 for a representative group $g$. The probability, $P_{i t g}(k)$, that player $i$ in group $g$ chooses $k$ is then

$$
P_{i t g}(k)=\frac{1}{z_{i t g}} e^{b_{t} U\left(x_{i k t g} I_{t} \bar{P}_{t g}^{e}(k)\right)}
$$

where $i, k$, and $g$ take the integer index values from 1 to $I$, $N_{t}$, and $G$, respectively. The expected value, $\bar{P}_{i t g}^{e}(k)$, denotes the belief, that is, the point expectation, that player $i$ in group $g$ holds on the average probability that $k$ is chosen within his or her group.

Suppose point expectations are homogeneous for all players in all G groups - that is, assume $\bar{P}_{i t g}^{e}(k)=\bar{P}_{t}(k)$. Then we have, further assuming that all covariate vectors 
Response/Bentley et al.: Mapping collective behavior

are homogeneous across all players and all groups,

$$
P_{t}(k)=P_{i t}(k)=\frac{1}{z_{t}} e^{b_{t} U\left(x_{k t}, t \bar{t}_{t}(k)\right)}
$$

The fixed point $\bar{P}^{*}(k)=k$ of Equation 1.2, which we might consider a "norm" of collective behavior, has been more formally labeled as a "logistic quantal response equilibrium with social interactions" (Blume et al. 2011; Brock \& Durlauf 2001b; 2006; McKelvey \& Palfrey 1995). For example, say person $i$ has both individual preferences concerning choice $k$ and forward-looking expectations concerning the popularity of choice $k$. Combining these influences as $a_{k}+c_{k}{ }^{\prime} X_{i}+d_{k}{ }^{\prime} \bar{X}_{g}+J_{k} \bar{P}_{g}(k)$, the probability that person $i$ will choose item $k$ is given by

$$
P(k)=P_{i}(k)=\frac{1}{z_{g}} e^{b\left(a_{k}+c_{k}^{\prime} X_{i}+d_{k}^{\prime} \bar{X}_{g}+J_{k} \bar{P}_{g}(k)\right)} .
$$

In Equation 1.3, each player $i$ has a covariate vector, $X_{i}$, and $\bar{X}_{g}$ denotes the average covariate vector averaged over players in $i$ 's group g. A fixed point of Equation 1.3 is a generalization of Nash equilibrium for discrete-choice games, and multiple Nash equilibria may arise when $b \geq 0$ and $J_{k} \geq 0$ for all $k$ choices (Brock \& Durlauf 2006).

At the extreme north of our map, $b=\infty$, and there can be many fixed points when the $J$ s are positive. A full analysis is beyond the scope of our response here (see Brock \& Durlauf 2006), but if we consider just the binary case, with $N=2$ (Brock \& Durlauf 2001a), we can fully characterize the set of fixed points for the case $J_{1}=J_{2}=J$. For the case of non-negative social interactions, $J \geq 0$, there can be three equilibria in many cases when $b J>1$ (e.g., in the northeast), if the difference among payoffs is not too large. MacCoun's framework is similarly based on binary decisions; hence his "balance of pressures" (BOP) model and the BOB model apply to the kinds of tipping points and "voter" outcomes that MacCoun mentions, including threshold effects.

Another good representation of the north-south axis is "noise," as suggested by Swain et al. with noise increasing as we move south. This noise dimension is what the socialphysics literature of fairly deterministic "preferential attachment" network models has yet to engage with. As a case in point, Fortunato et al. argue that in a fully connected network, the random imitation of the southeast and popularity-based choice of the northeast (we actually map conformity in the equatorial east) are the same, through preferential attachment. But this neglects the greater degree of random noise as we move south, and hence the noise in choice popularity. Whereas the highly right-skewed distribution does not change much moving down the eastern edge of the map, as Analytis et al. also mention, the dynamics do change. This is the point of our Figure 2b.

To explore what happens with the greater noise in the south, let us examine equilibria for the extreme south, where $b=0$. We have $P(k)=1 / N$, where $N$ is the number of possible choices, that is, all players are just making choices at random, with the probability of each choice equal to $1 / N$. When we are in the deep south, where $b$ approaches 0 , we see that no matter how strong social ties are, that is, no matter how large $J$ is, there is only one equilibrium. However, when we are in the extreme north, where $b$ is very large, there will be, in many cases, three equilibria in the extreme northeast but only one equilibrium in the extreme northwest. In the extreme southeast, where $J$ is very large, a small value of $b$ can still satisfy $b J>1$, so multiple equilibria can easily occur.

\section{R4. The future of big data}

The southeast is where we find the unpredictability of success that Watts, Salganik, and colleagues have revealingly demonstrated over the years (e.g., Salganik et al. 2006; Watts \& Hasker 2006). This underlies our main question concerning big data: Will the popularity of crowd sourcing soon undo itself, decreasing $b$ through information overload while simultaneously increasing social awareness of the crowd, $J$, and hence move online society toward the southeast? Slow movement of the key parameters $(b, J)$ from west to east and from north to south, but especially from northwest to southeast, can easily produce behavior such as bifurcations and phase transitions, as unique equilibria morph into multiple equilibria (Berry et al. 1995; Brock \& Durlauf 2001a; 2006). Hence, Moat et al., who describe remarkable discoveries of the predictive nature of big data, may soon need to consider future increases in social-interaction effects as those predictive methods become commonplace.

We are grateful to Christen \& Brugger for contributing a historical perspective through their invocation of Hacking's (1992; 1995) principle of "looping," in which the identification of a phenomenon then feeds into the phenomenon itself. This is exactly why we believe that as predictive as big data might be at the moment, as soon as everyone becomes aware of these predictive algorithms, the competition to outpredict your competitor-whether in fashion, business, or the like-will tend to increase the unpredictability, much like what we see in the stock market. For example, whereas natural systems tend to exhibit early warning systems before critical transitions, financial systems are much more elusive (Scheffer et al. 2012).

Fan \& Suchow propose that self-awareness can lead a group to seek out new knowledge and reposition itself on the map. They propose that motivation to solve a problem is a key variable driving groups northward on the map. We are not sure that the crowd can guide its own trajectory, however. We all seek to head north, but as Schmidt points out, the onslaught of big data may break lots of compasses, given that practically any opinion on any issue from climate change to genetically modified foods to measles vaccines and evolution can be found online. As Schmidt points out, even human identity becomes ambiguous as each person's digital shadow grows with multiple memberships, enrollments, sign-ups, connections, and so on. Intriguingly, Schmidt proposes that the southeast may subdue the data deluge "through a global relativity," while at the same time making the problem worse through collective behaviors. Schmidt rightly asks whether any of us can be experts anymore, which poses the compelling question of what happens if we were to crowd-source all our decisions, as is already explored in current science fiction. Schmidt suggests this may already be happening in medicine, perhaps the most informationdeluged science, where the diagnosis of newly named syndromes has been rising dramatically in a way that is clustered in time and space, suggestive of the southeast.

As O'Donnell et al. point out, the neural systems for self-knowledge are involved in social cognition. In fact, 
Christen \& Brugger add self-reflection to the list of suggested third dimensions to the map because social influence depends on the models people have of themselves and what drives their behavior (e.g., a conscious effort to be a nonconformist) and, of course, whether one is aware of this self-reflection (buck the nonconformist trend by being ironically conformist, for example). This looping can go on forever, reminiscent of what Yogi Berra once said about a popular Italian restaurant in St. Louis: "No one goes there anymore. It's too crowded."

As agents compete to outpredict each other, we enter Mesoudi's discussion of adaptive landscapes (see also Mesoudi 2010; Mesoudi \& O’Brien 2008a; 2008b). Anderson et al. (1992) give us a formula for this:

$$
\operatorname{Emax}_{i \varepsilon\left\{1,2, \ldots, N_{t}\right\}}\{\tilde{U}\}=\frac{1}{b_{t}} \ln \left(\sum_{i=1}^{N_{t}} e^{b_{t} U_{i}}\right)
$$

which relates to ideas and techniques from entropy maximization in Bayesian statistics - tools from mean-field theory that allow us to approximate more complicated social networks, economics, and finance (Ben-Akiva et al. 2012; Brock 1993; Hommes 2013). Note that as $b_{t} \rightarrow \infty$ from south to north, this landscape function converges to $U_{i^{*}}, i^{*}=\operatorname{argmax}_{k}\left\{U_{k}\right\}$ and hence the landscape of Equation 1.4 moves toward a spiked, or so-called "Mount Fuji," shape on the space of choices, $\left\{1,2, \ldots, N_{t}\right\}$. In contrast, moving from west to east, as $J_{t}$ increases, multiple equilibria become possible so the landscape becomes more "rugged," and the potential for instability grows. Generally speaking, instabilities in collective dynamics will be north of the "equator," and the northeast especially is where instabilities and emergent bifurcations could link to studies of early warning signals (Scheffer et al. 2012).

Roesch et al. mention the "exponential velocity" of change with access to new technology, but we need to be careful about tempo versus mode of change, which might lead us to ask whether the current rate of change is any more "exponential" than in the early twentieth century, with most technologies taking off in the same sigmoidal form (Bentley \& O'Brien 2012; O'Brien \& Bentley 2011). Rather than accelerating change, in some cases increasing access to crowd-sourced data may lead to stasis, especially if accurate popularity statistics are approached with a conformity bias.

On an adaptive fitness landscape, such as what Mesoudi describes, there are different ways to avoid getting stuck on a low peak when higher peaks are nearby. The most straightforward is a global view of the landscape from the authoritative vantage of a "control tower," which is the beauty of the Sixth Sense Transport system that Norgate et al. have developed for mitigating traffic problems. Without this kind of top-down control, the other means is an optimal balance of information producers and information scroungers, as Mesoudi would describe it (see also Mesoudi 2008), or a balance of "exploration and exploitation," in the more business-like language of Axelrod and Cohen (1999).

On our map, this means an optimal, situation-specific balance of noise (the opposite of transparency) and social influence. Clearly, the balance is critical. Norgate et al. point out that "increased imitation can be desirable," and Zhou et al. note that when people have little knowledge on which to base their decisions, it is helpful to imitate the successful judgments, or to average the judgments, of others. But under a deluge of information, imitation might also bring about the kind of "pluralistic ignorance" that Christen \& Brugger suggest could stabilize social dynamics in a suboptimal state. Crowd-sourcing may even preserve an undesirable status quo. As Uhlmann \& Silberzahn discuss, we assume that the best person for the job gets hired - the northwest - but actual online hiring decisions tend to drift southeast, as information overload leads employers to rely on gender stereotypes as a shortcut decision strategy.

As a result, noise can be a means of (unintentionally) exploring the landscape, as nicely shown by Hopfensitz et al. with their game involving ambiguous payoffs (lesstransparent payoffs in our language) and strong social interactions, which can yield a more efficient outcome than the same game with less of those aspects in the north. Noise in the dynamics of the game can lead to coordination equilibrium with a higher level of social welfare when multiple Nash equilibria are present in coordination games (Kandori et al. 1993). Hopfensitz et al. formulate their first game so that each player who has ties to another player chooses his or her strategy to maximize a combined utility that is a weighted sum of his own selfish interest and the joint interest of the two players. If the function $U$ in BOB's Equation 1 is replaced by the Hofensitz et al. function, and $b=0$, then the players will choose their strategies randomly with equal probability, and yet, as Hopfensitz et al. point out, this may yield a higher average welfare than in the case where $b$ is large.

O'Donnell et al. and Pfister \& Böhm both object to the idea of "independence" as a possibility for human beings, whose minds are social, even when physically alone. They point to neuroimaging studies that show humans are prone to adopt ideas that they may think are becoming more popular (see also Berger \& Le Mens 2009; Berger \& Milkman 2012; Gureckis \& Goldstone 2009), even if they happen to be alone at the time. As O'Donnell et al. note, the neural systems for self-knowledge are involved in social cognition, and social inclusion versus rejection involves the same areas of the brain as physical pleasure and pain. As compelling as the evidence for the "social brain" may be, this is more of a semantic issue for us, to be plotted on the map using data at the scale of individuals or populations.

As Moat et al. correctly point out, we were not generous enough to the new big-data sciences of predicting nearfuture behavior based on recent past behavior. Roesch et al. and Moat et al. report on the incredible progress in data generation concerning individual behaviors correlated with all the other geolocated measures one can derive such as ambient temperature, noise level, luminance information, and energy consumption. Our review was only the tip of the iceberg in terms of big-data studies aimed at forecasting future behavior such as financial and commercial activity, economic trends, epidemics, and even crime. As behavioral scientists, we are in awe of the developments in e-commerce, such as recommendation systems in retail applications. As a clarification, we would see computer algorithms recommending products or services as probably qualifying as social influence, even if the recommendation algorithms are using social information to act on people who may be alone at their computers (an ambiguity raised by O'Donnell et al.).

Predicting the near future by extrapolating the recent past is a huge advantage of big data, especially because it does not require deep understanding of the causality 
References/Bentley et al.: Mapping collective behavior

behind behaviors in terms of decision making. Causality is enigmatic in the same way that social diffusion (northeast) can be so difficult to distinguish from (northwest) homophily (Aral et al. 2009), but for prediction and intervention, this may make little difference. The prescription is to intervene at the point of highest activity, with or without knowledge of its cause. Moat et al. make the important point that not only can big data assist in "predicting the present" (Choi \& Varian 2012), but it can also play a major role in predicting the future-for example, in the trading-strategy-returns studies that they cite.

Although our map is an extremely coarse-grained approximation of the highly dimensioned correlation and prediction studies that Moat et al. cite, we show here how the $b_{t}$ index of "transparency" relates to those studies. Note that

$$
\ln \left(\frac{P_{t}(k)}{P_{t}\left(N_{t}\right)}\right)=b_{t}\left(U_{k t}-U_{N_{t}, t}\right) .
$$

In applications of discrete-choice theory to correlation and prediction, the $U s$ are parameterized as functions of observable covariates and parameters and taken to datasets for hypothesis formulation, estimation of parameters, and testing of hypotheses. This activity includes correlation studies and prediction studies. If $b_{t}=0$, the differences in the estimated values of the $U s$ give no information as to the observed frequencies of choices between choice $k$ and choice $N_{t}$ at date $t$. In this case, the covariates give no information on predicting the present or the future. More concretely in the Preis et al. (2013) study that Moat et al. cite, we could think of big-data sets from Google Trends as a way of not only improving the specification of the $U_{s}$ in Equation 1.5, but also of increasing the size of $b_{t}$ relative to previous studies.

To be sure, this may soon be less painstaking with better data and algorithms based on pioneering studies by Aral and Walker (2012) and others, at which point the map will be even more appropriate because measuring the east-west dimension might be routine. Mapping the nature of decisions is crucial because big data will soon be part of our decisions rather than an independent measure of them. Roesch et al. mention Project Glass (Google Inc. 2012) and the ubiquity of big data in daily activities, although we note that still only about a third of the world's population has Internet access. As the growing public familiarity with big-data patterns feeds into the decisions themselves Christen \& Brugger; Fan \& Suchow; Schmidt), will big data still be as predictive (Moat et al.), or are we heading toward a situation, as with financial markets, where everyone is trying to outpredict everyone else? How will collective behavior change as we all become omniscient about global trends in those very behaviors? Buck is correct to discuss McLuhan's (1964) "medium is the message" philosophy, which underlies our basic question of how big data will change behavior and not merely record it objectively.

In conclusion, we again want to thank all the commentators for providing us much more in the way of excellent proposals for modifying and extending the BOB map into areas that we could not have anticipated. Our only regret is not having the time and the space to respond to the comments in the terms they deserve. We hope both our target article and the accompanying commentaries will inspire other behavioral scientists with an interest in decision making to use the discussions as launching pads for their own work. We very much look forward to what those others have to say.

\section{References}

[The letters “ $a$ " and " $r$ " before author's initials stand for target article and response references, respectively]

Acerbi, A., Lampos, V., Garnett, P. \& Bentley, R. A. (2013) The expressions of emotions in 20th century books. PLOS ONE 8(3):e59030. [rRAB]

Adamic, L. A. \& Huberman, B. A. (2000) Power-law distribution of the World Wide Web. Science 287:2115. [aRAB]

Aïte, A., Cassotti, M., Rossi, S., Poirel, N., Lubin, A., Houdé, O. \& Moutier, S. (2012) Is human decision making under ambiguity guided by loss frequency regardless of the costs? A developmental study using the Soochow Gambling Task. Journal of Experimental Child Psychology 113:286-94. [SMR]

Akerlof, G. A. \& Shiller, R. (2009) Animal spirits: How human psychology drives the economy, and why it matters for global capitalism. Princeton University Press. [H-RP]

Albert, R. \& Barabási, A. L. (2002) Statistical mechanics of complex networks. Reviews of Modern Physics 74(1):47-97. [JES]

Aldrich, D. P. (2012) How to weather a hurricane. New York Times, August 2, 2012 , p. A27. [aRAB]

Allen, D. \& Wilson, T. D. (2003) Information overload: Context and causes. New Review of Information Behaviour Research 4:31-44. [aRAB]

Allen, P. M. \& McGlade, J. M. (1986) Dynamics of discovery and exploitation: The case of the Scotian Shelf groundfish fisheries. Canadian Journal of Fisheries and Aquatic Science 43:1187-200. [aRAB]

Alleva, L. (2006) Taking time to savour the rewards of slow science. Nature 443 (7109):271. [CTS]

Alvergne, A., Gurmu, E., Gibson, M. A. \& Mace, R. (2011) Social transmission and the spread of modern contraception in rural Ethiopia. PLoS ONE 6(7): e22515. [aRAB]

Alves, R. R. N. \& Rosa, I. M. L. (2007) Biodiversity, traditional medicine and public health: Where do they meet? Journal of Ethnobiology and Ethnomedicine 3:14. [aRAB]

Amaral, L. A. N., Scala, A., Barthélémy, M. \& Stanley, H. E. (2000) Classes of smallworld networks. Proceedings of the National Academy of Sciences USA 97:11149-52. [aRAB]

Amaro de Matos, J. \& Perez, J. (1991) Fluctuations in the Curie-Weiss version of the random field Ising model. Journal of Statistical Physics 6:587-608. [aRAB]

Anderson S., de Palma, A. \& Thisse, J. F. (1992) Discrete choice theory of product differentiation. MIT Press. [rRAB]

Angner, E. (2012) A course in behavioral economics. Palgrave Macmillan. [DRo]

Angner, E. \& Loewenstein, G. (2012) Behavioral economics. In: Philosophy of economics, ed. U. Mäki, pp. 641-89. Elsevier. [DRo]

Apicella, C. L., Marlowe, F. W., Fowler, J. H. \& Christakis, N. A. (2012) Social networks and cooperation in hunter-gatherers. Nature 481:497-501. [aRAB]

Aral, S. \& Walker, D. (2012) Identifying influential and susceptible members of social networks. Science 337:337-41. [arRAB]

Aral, S., Muchnik, L. \& Sundararajan, A. (2009) Distinguishing influence-based contagion from homophily-driven diffusion in dynamic networks. Proceedings of the National Academy of Sciences USA 106:21544-49. [rRAB]

Arawatari, R. (2009) Informatization, voter turnout and income inequality. Journal of Economic Inequality 7:29-54. [aRAB]

Archer, M. S. \& Tritter, J. Q. (2000) Introduction. In: Rational choice theory. Resisting colonization, ed. M. S. Archer \& J. Q. Tritter, pp. 1-16. Routledge. [ANG]

Ariely, D. (2008) Predictably irrational: The hidden forces that shape our decisions. Harper Collins. [H-RP, DRo]

Arthur, W. (1989) Competing technologies, increasing returns, and lock-in by historical events. The Economic Journal 99:116-31. [GLM]

Artinger, F. \& Gigerenzer, G. (in preparation) Pricing in an uncertain market. Mimeo. [PPA]

Asch, S. E. (1951) Effects of group pressure on the modification and distortion of judgements. In: Groups, leadership and men, ed. H. Guetzkow, pp. 177-90. Carnegie Press. [XZ]

Asch, S. E. (1952) Social psychology. Prentice-Hall. [XZ]

Asch, S. E. (1956) Studies of independence and conformity: I. A minority of one against a unanimous majority. Psychological Monographs: General and Applied $70(9): 1-70 . \quad[\mathrm{XZ}]$

Askitas, N. \& Zimmermann, K. F. (2009) Google econometrics and unemployment forecasting. Applied Economics Quarterly 55:107-20. [HSM] 
Asur, S. \& Huberman, B. A. (2010) Predicting the future with social media. In: Proceedings of the 2010 IEEE/WIC/ACM International Conference on Web Intelligence and Intelligent Agent Technology (WI-IAT), pp. 492-99. IEEE. [MTK]

Atkisson, C., O’Brien, M. J. \& Mesoudi, A. (2012) Adult learners in a novel environment use prestige-biased social learning. Evolutionary Psychology 10:519-37. [aRAB]

Attanasi, G., Hopfensitz, A., Lorini, E. \& Moisan, F. (2013) The behavioral effects of social ties when their subjective and objective strength vary. Toulouse School of Economics Working Paper. [AH]

Aumann, R. J. (2003) Presidential address. Games and Economic Behavior 45:214. [RAM $]$

Avnet, T. \& Sellier, A.-L. (2011) Clock time vs. event time: Temporal culture or selfregulation? Journal of Experimental Social Psychology 47:665-67. doi: 10.1016/ j.jesp.2011.01.006. [SHN]

Axelrod, R. \& Cohen, M. D. (1999) Harnessing complexity: Organizational implications of a scientific frontier. Free Press. [rRAB]

Bagozzi, R. P., Gopinath, M. \& Nyer, P. U. (1999) The role of emotions in marketing. Journal of the Academy of Marketing Science 27:184-206. doi: 10.1177/ 0092070399272005 . [ANG]

Bakshy, E., Hofman, J. M., Mason, W. A. \& Watts, D. J. (2011) Everyone’s an influencer: Quantifying influence on Twitter. In: WSDM'11: Proceedings of the 4th International ACM Conference on Web Search and Data Mining, Hong Kong, China, February 9-12, 2011, ed. I. King, W. Nejdl, \& H. Li, pp. 65-74. ACM Digital Library. [MBO]

Balcan, D., Colizza, V., Gonçalves, B., Hu, H., Ramasco, J. J. \& Vespignani, A. (2009) Multiscale mobility networks and the spatial spreading of infectious diseases. Proceedings of the National Academy of Sciences USA 106:21484-89. [HSM]

Bandelj, N. (2009) Emotions in economic action and interaction. Theory and Society 38:347-66. [ANG]

Barabási, A.-L. \& Albert, R. (1999) Emergence of scaling in random networks. Science 286(5439):509-12. Available at: http://www.ncbi.nlm.nih.gov/pubmed/ 10521342. [aRAB, SF, JES]

Baron, J. (2007) Thinking and deciding, 3rd and 4th editions. Cambridge University Press. [aRAB, H-RP]

Basalla, G. (1989) The evolution of technology. Cambridge University Press. [aRAB]

Bates, B. R., Romina, S., Ahmed, R. \& Hopson, D. (2006) The effect of source credibility on consumers' perceptions of the quality of health information on the Internet. Medical Informatics and the Internet in Medicine 31:45-52. [aRAB]

Batty, M. (2006) Rank clocks. Nature 444:592-96. [aRAB]

Baumeister, R. F. \& Tierney, J. (2011) Willpower: Rediscovering the greatest human strength. Penguin. [aRAB]

Bechara, A. \& Damasio, A. R. (2005) The somatic marker hypothesis: A neural theory of economic decision. Games and Economic Behavior 52:336-72. [MT]

Becker, G. S. (1962) Irrational behavior and economic theory. Journal of Political Economy 70:1-13. [aRAB]

Becker, G. S. (1976) The economic approach to human behavior. University of Chicago Press. [aRAB]

Becker, G. S. (1991) A treatise on the family, enlarged edition. Harvard University Press. [aRAB]

Behrens, T. E. \& Sporns, O. (2012) Human connectomics. Current Opinion in Neurobiology 22(1):144-53. Available at: http://www.ncbi.nlm.nih.gov/pubmed/ 21908183 [JES]

Beinhocker, E. D. (2006) The origin of wealth: evolution, complexity, and the radical remaking of economics. Random House. [aRAB]

Belefant-Miller, H. \& King, D. W. (2001) How, what, and why science faculty read. Science and Technology Libraries 19:91-112. [aRAB]

Ben-Akiva, M., de Palma, A., McFadden, D., Abou-Zeid, M., Chiappori, P-A., de Lapparent, M., Durlauf, S. N., Fosgerau, M., Fukuda, D., Hess, S., Manski, C., Pakes, A., Picard, N. \& Walker, J. (2012) Process and context in choice models. Marketing Letters 23:439-56. [rRAB]

Benotsch, E. G., Kalichman, S. \& Weinhardt, L. S. (2004) HIV-AIDS patients' evaluations of health information on the Internet: The digital divide and vulnerability to fraudulent claims. Journal of Consulting and Clinical Psychology 72:1004-11. [aRAB]

Bentley, R. A., Earls, M. \& O’Brien, M. J. (2011) I'll have what she's having: Mapping social behavior. MIT Press. [aRAB]

Bentley, R. A., Garnett, P., O’Brien M. J. \& Brock, W. A. (2012) Word diffusion and climate science. PLoS ONE 7(11):e47966. [arRAB, JEF, MTK]

Bentley, R. A., Hahn, M. W. \& Shennan, S. J. (2004) Random drift and culture change. Proceedings of the Royal Society of London B 271:1443-50. [AM]

Bentley, R. A., Lipo, C. P., Hahn, M. W. \& Herzog, H. A. (2007) Regular rates of popular culture change reflect random copying. Evolution and Human Behavior 28:151-58. [aRAB]

Bentley, R. A. \& Maschner, H. D. G. (2000) A growing network of ideas. Fractals 8:227-37. [aRAB]
Bentley, R. A. \& O’Brien, M. J. (2011) The selectivity of social learning and the tempo of cultural evolution. Journal of Evolutionary Psychology 9:125-41. [aRAB]

Bentley, R. A. \& O’Brien, M. J. (2012) The buzzwords of the crowd. New York Times, December 1, 2012, p. SR4. [arRAB]

Bentley, R. A. \& Ormerod, P. (2010) A rapid method for assessing social versus independent interest in health issues: A case study of "bird flu" and "swine flu". Social Science and Medicine 71(3):482-85. [aRAB, MTK]

Beppu, A. \& Griffiths, T. L. (2009) Iterated learning and the cultural ratchet. In: Proceedings of the 31st Annual Conference of the Cognitive Science Society. ed. N. A. Taatgen \& H. van Rijn, pp. 2089-94. Cognitive Science Society. [SMR, $\mathrm{DRu}]$

Berezin, M. (2005) Emotions and the economy. In: Handbook of economic sociology, 2nd edition, ed. R. Swedberg \& N. J. Smelser, pp. 109-27. Russell Sage Foundation and Princeton University Press. [ANG]

Berezin, M. (2009) Exploring emotions and the economy: New contributions from sociological theory. Theory and Society 38:335-46. [ANG]

Berger, J. \& Le Mens, G. (2009) How adoption speed affects the abandonment of cultural tastes. Proceedings of the National Academy of Sciences USA 106:814650. [arRAB]

Berger, J. \& Milkman, K. L. (2012) What makes online content viral? Journal of Marketing Research 49:192-205. [MBO, rRAB]

Berry, S., Levinsohn, J. \& Pakes, A. (1995) Automobile prices in market equilibrium. Econometrica 63:841-90. [rRAB]

Bettencourt, L. M. A., Lobo, J., Helbing, D., Kühnert, C. \& West, G. B. (2007a) Growth, innovation, scaling, and the pace of life in cities. Proceedings of the National Academy of Sciences USA 104:7301-6. [aRAB]

Bettencourt, L. M. A., Lobo, J. \& Strumsky, D. (2007b) Invention in the city: Increasing returns to patenting as a scaling function of metropolitan size. Research Policy 36:107-20. [aRAB]

Bettinger, R. L., Boyd, R. \& Richerson, P. J. (1996) Style, function and cultural evolutionary processes. In: Darwinian archeologies, ed. H. D. G. Maschner, pp. 133-62. Plenum Press. [aRAB]

Bhasker, B. \& Srikumar, K. (2010) Recommender systems in e-commerce. Cambridge University Press. [EBR]

Bidwell, M., Briscoe, F., Fernandez-Mateo, I. \& Sterling, A. (2013) The employment relationship and inequality: How and why changes in employment practices are reshaping rewards in organizations. The Academy of Management Annals 7 (1):61-121. [ELU]

Biermann, J. S., Golladay, G. J., Greenfield, M. L. V. H. \& Baker, L. H. (1999) Evaluation of cancer information on the Internet. Cancer 86:381-90. [aRAB]

Bikhchandani, S., Hirshleifer, D. \& Welch, I. (1992) A theory of fads, fashion, custom, and cultural change as informational cascades. Journal of Political Economy 100(5):992-1026. [GLM]

Binder, K. \& Young, A. P. (1986) Spin glasses: Experimental facts, theoretical concepts, and open questions. Reviews of Modern Physics 58(4):801-976. [JES]

Block, N. (2007) Consciousness, accessibility, and the mesh between psychology and neuroscience. Behavioral and Brain Sciences 30:481-48. [EJG]

Blume, L. E., Brock, W. A. Durlauf, S. N. \& Ioannides, Y. M. (2011) Identification of social interactions. In: Social economics, ed. J. Benhabib, M. O. Jackson \& A. Bisin, pp. 853-964. North-Holland. [rRAB]

Bodenhausen, G. V. (1988) Stereotypic biases in social decision making and memory: Testing process models of stereotype use. Journal of Personality and Social Psychology 55(5):726-37. [ELU]

Bodenhausen, G. V. (1990) Stereotypes as judgmental heuristics: Evidence of circadian variations in discrimination. Psychological Science 1:319-22. [ELU]

Böhm, G. \& Pfister, H.-R. (2000) Action tendencies and characteristics of environmental risks. Acta Psychologica 104(3):317-37. [H-RP]

Bonet, R., Cappelli, P. \& Hamori, M. (2013) Labor market intermediaries and the new paradigm for human resources. The Academy of Management Annals $7(1): 339-90 . \quad$ [ELU]

Bordieu, P. (1990) The logic of practice. Stanford University Press. [aRAB]

Borgatti, S. P., Mehra, A., Brass, D. J. \& Labianca, G. (2009) Network analysis in the social sciences. Science 323:892-95. [aRAB]

Bowers, K. J., Johnson, S. \& Pease, K. (2004) Prospective hotspotting: The future of crime mapping? British Journal of Criminology 44:641-58. [HSM]

Boyd, R. \& Richerson, P. J. (1985) Culture and the evolutionary process. University of Chicago Press. [AM, SMR]

Bramoullé, Y. (2007) Anti-coordination and social interactions. Games and Economic Behavior 58:30-49. [rRAB]

Brehmer, B. (1980) In one word: Not from experience. Acta Psychologica 45:223-41. [H-RP]

Brock, W. A. (1993) Pathways to randomness in the economy: Emergent nonlinearity and chaos in economics and finance. Estudios Economicos 8 (1):3-55. [rRAB]

Brock, W. A. \& Durlauf, S. N. (1999) A formal model of theory choice in science. Economic Theory 14:113-30. [aRAB, EBR] 
Brock, W. A. \& Durlauf, S. N. (2001a) Discrete choice with social interactions. Review of Economic Studies 68:229-72. [rRAB]

Brock, W. A. \& Durlauf, S. N. (2001b) Interactions-based models. In: Handbook of econometrics, vol. 5, ed. J. Heckman \& E. Leamer, pp. 3297-80. Elsevier Science. [arRAB]

Brock, W. A. \& Durlauf, S. N. (2006) Multinomial choice with social interactions. In: The economy as an evolving complex system, ed. L. E. Blume \& S. N. Durlauf, pp. 175-206. Oxford University Press. [rRAB]

Brock, W. A. \& Durlauf, S. N. (2010) Adoption curves and social interactions. Journal of the European Economic Association 8:232-51. [rRAB]

Brownstein, J. S., Freifeld, C. C. \& Madoff, L. C. (2009) Digital disease detection harnessing the web for public health surveillance. New England Journal of Medicine 360:2153-57. [HSM]

Buck, R., Anderson, E., Chaudhuri, A. \& Ray, I. (2004) Emotion and reason in persuasion: Applying the ARI model and the CASC scale. Journal of Business Research. Marketing Communications and Consumer Behavior 57:647-56. [RB]

Buck, R. \& Ferrer, R. (2012) Emotion, warnings, and the ethics of risk communication. In: Handbook of risk theory, ed. S. Roeser, R. Hillerbrand, P. Sandin \& M. Peterson, pp. 694-723. Springer. [RB]

Buck, R. \& Powers, S. R. (2011) Emotion, media, and the Global Village. In: The Routledge handbook of emotions and mass media, ed. K. Doveling, C. von Scheve \& E. A. Konijn, pp. 181-94. Routledge. [RB]

Bullmore, E. \& Sporns, O. (2012) The economy of brain network organization. Nature Reviews Neuroscience 13(5):336-49. Available at: http://www.ncbi.nlm. nih.gov/pubmed/22498897 [JES]

Burling, R. (1993) Primate calls, human language, and nonverbal communication. Current Anthropology 34:25-53. [aRAB]

Burton, M., Rigby, D., Young, T. \& James, S. (2001) Consumer attitudes to genetically modified organisms in food in the UK. European Review of Agricultural Economics 28(4):479-98. [JEF]

Busemeyer, J. R. \& Johnson, J. G. (2006) Micro-process models of decision-making. In: Cambridge handbook of computational cognitive modeling, ed. R. Sun, pp. 302-21. Cambridge University Press. [DRu]

Byrne, R. W. \& Russon, A. E. (1998) Learning by imitation: A hierarchical approach. Behavioral and Brain Sciences 21:667-721. [aRAB]

Caldwell, C. A. \& Whiten, A. (2002) Evolutionary perspectives on imitation: Is a comparative psychology of social learning possible? Animal Cognition 5:193208. [aRAB]

Camerer, C., Loewenstein, G. \& Rabin, M., eds. (2004) Advances in behavioral economics. Russell Sage Foundation. [H-RP]

Canguilhem, G. (1966) Le normal et le pathologique. Presses Universitaires de France. [CTS]

Cappelli, P. \& Keller, J. R. (in press) Classifying work in the new economy. Academy of Management Review. [ELU]

Carlsson, H. \& van Damme, E. (1993) Global games and equilibrium selection. Econometrica 61:989-1018. [DRo]

Carr, N. (2008) Is Google making us stupid? The Atlantic, July/August 2008, pp. 56-63. [aRAB]

Castellano, C., Fortunato, S. \& Loreto, V. (2009) Statistical physics of social dynamics. Reviews of Modern Physics 81:591-46. [SF]

Cavagna, A., Cimarelli, A., Giardina, I., Parisi, G., Santagati, R., Stefanini, F. \& Viale, M. (2010) Scale-free correlations in starling flocks. Proceedings of the National Academy of Sciences USA 107:11865-70. [SMR]

Cavalli-Sforza, L. L. \& Feldman, M. W. (1981) Cultural transmission and evolution Princeton University Press. [aRAB, AM]

Centola, D. (2010) The spread of behavior in an online social network experiment Science 329:1194-97. [aRAB, SF]

Cha, M., Benevenuto, F., Haddadi, H. \& Gummadi, K. P. (2012) The world of connections and information flow in Twitter. IEEE Transactions on Systems, Man and Cybernetics, Part A: Systems and Humans 42:991-98. [aRAB]

Cha, M., Haddadi, H., Benevenuto, F. \& Gummadi, K. P. (2010) Measuring user influence in Twitter: The million follower fallacy. In: Proceedings of the Fourth International AAAI Conference on Weblogs and Social Media, ed. M. A. Hearst W. Cohen \& S. Gosling, pp. 10-17. AAAI Press. [aRAB]

Chapman, B. B., Ward, A. J. W. \& Krause, J. (2008) Schooling and learning: Early social environment predicts social learning ability in the guppy, Poecilia reticulata. Animal Behaviour 76:923-29. [SMR]

Chaudhuri, A. \& Buck, R. (1995) Media differences in rational and emotional responses to advertising. Journal of Broadcasting and Electronic Media 39(1):109-25. [RB]

Choi, H. \& Varian, H. (2012) Predicting the present with Google Trends. Economic Record 88(Suppl. s1):2-9. [MTK, HSM, rRAB]

Christakis, N. A. \& Fowler, J. H. (2007) The spread of obesity in a large social network over 32 years. New England Journal of Medicine 357:370-79. [aRAB, MBO]

Christen, M., Alfano, M., Bangerter, B. \& Lapsley, D. (2013) Ethical issues of morality mining: When the moral identity of individuals becomes a focus of data
References/Bentley et al.: Mapping collective behavior

mining. In: Ethical data mining applications for socio-economic development, ed. H. Rahman \& I. Ramos, pp. 1-21. IGI Global. [MC]

Christensen-Szalanski, J. J. J. (1978) Problem solving strategies: A selection mechanism, some implications, and some data. Organizational Behavior and Human Performance 22:307-23. [ELU]

Christensen-Szalanski, J. J. J. (1980) A further examination of the selection of problem-solving strategies: The effects of deadlines and analytic aptitudes Organizational Behavior and Human Performance 25:107-22. [ELU]

Clauset, A., Shalizi, C. R. \& Newman, M. E. J. (2009) Power-law distributions in empirical data. SIAM Review 51:661-703. [aRAB]

Colander, D., ed. (1996) Beyond microfoundations. Cambridge University Press. [DRo]

Conner, A., Glare, T. \& Nap, J. (2003) The release of genetically modified crops into the environment. The Plant Journal 33(1):19-46. [JEF]

Couzin, I. D., Ioannou, C. C., Demirel, G., Gross, T., Torney, C. J., Hartnett, A., Conradt, L., Levin, S. A. \& Leonard, N. E. (2011) Uninformed individuals promote democratic consensus in animal groups. Science 334:1578-80. [aRAB]

Couzin, I. D., Krause, J., Franks, N. R. \& Levin, S. A. (2005) Effective leadership and decision making in animal groups on the move. Nature 433:513-16. [aRAB]

Cox, J. \& Harrison, G., eds. (2008) Risk aversion in experiments. Emerald Group. [DRo]

Dake, K. (1991) Orienting dispositions in the perception of risk: An analysis of contemporary worldviews and cultural biases. Journal of Cross-Cultural Psychology 22:61-82. [SHN]

Dall, S. R. X. \& Johnstone, R. A. (2002) Managing uncertainty: Information and insurance under the risk of starvation. Philosophical Transactions of the Royal Society B 357:1519-26. [SMR]

Damasio, A. R. (1994) Descartes' error: Emotion, reason and the human brain. Putnam Press. [H-RP]

Darley, J. M. \& Gross, P. H. (1983) A hypothesis-confirming bias in labeling effects Journal of Personality and Social Psychology 44:20-33. [ELU]

Davis, A. \& Fu, D. (2004) Culture matters: Decision making through increased awareness. Interservice/Industry Training, Simulation, and Education Conference Paper, No. 1852, pp. 1-9. [aRAB]

Dawson, E. H., Avargues-Weber, A., Chittka, L. \& Leadbeater, E. (2013) Learning by observation emerges from simple associations in an insect model. Current Biology 23:727-30. [SMR]

Day, R. L., MacDonald, T., Brown, C., Laland, K. N. \& Reader, S. M. (2001) Interactions between shoal size and conformity in guppy social foraging. Animal Behaviour 62:917-25. [SMR]

Decety, J. \& Sommerville, J. A. (2003) Shared representations between self and other: A social cognitive neuroscience view. Trends in Cognitive Sciences 7(12):527-33. [MBO]

Dell'Omo, G., Ricceri, L., Wolfer, D. P., Poletaeva, I. I. \& Lipp, H. P. (2000) Temporal and spatial adaptation to food restriction in mice under naturalistic conditions. Behavioural Brain Research 115:1-8. [SMR]

Dennett, D. (1995) Darwin's dangerous idea. Simon and Schuster. [aRAB]

Dennett, D. C. (1996) Kinds of minds: Towards an understanding of consciousness. Weidenfeld \& Nicolson. [DS]

Denrell, J. \& Le Mens, G. (2007) Interdependent sampling and social influence. Psychological Review 114(2):398-422. doi:10.1037/0033-295X.114.2.398. [GLM]

Denrell, J. \& Le Mens, G. (2013) Information sampling, conformity and collective mistaken beliefs. In: Proceedings of the 35th Annual Conference of the Cognitive Science Society, ed. M. Knauff, M. Pauen, N. Sebanz \& I. Wachsmuth, pp. 2177-82. Cognitive Science Society. [GLM]

de Sola Price, D. J. (1965) Networks of scientific papers. Science 149:510-15. [aRAB]

Deutsch, M. \& Gerard, H. B. (1955) A study of normative and informational social influences upon individual judgment. Journal of Abnormal and Social Psychology 51(3):629-36. [XZ]

DeVellis, R. F. (2012) Scale development: Theory and applications, 3rd edition. Sage. $[\mathrm{XZ}]$

Dodds, P. S. \& Watts, D. J. (2005) A generalized model of social and biological contagion. Journal of Theoretical Biology 232:587-604. [aRAB]

Dreber, A., Rand, D. G., Fudenberg, D. \& Nowak, M. A. (2008) Winners don't punish. Nature 452:348-51. [aRAB]

Dunbar, R. I. M. (1992) Neocortex size as a constraint on group size in primates Journal of Human Evolution 22:469-93. [aRAB]

Dunbar, R. I. M. (1993) Coevolution of neocortical size, group size and language in humans. Behavioral and Brain Sciences 16:681-735. [aRAB]

Dunbar, R. I. M. (1998) The social brain hypothesis. Evolutionary Anthropology 6:178-90. [aRAB]

Durkheim, E. (2001) The elementary forms of the religious life. Oxford University Press. $\quad[\mathrm{ANG}]$

Durlauf, S. (1999) How can statistical mechanics contribute to social science? Proceedings of the National Academy of Sciences USA 96:10582-84. [aRAB] 
Durlauf, S. (2002) On the empirics of social capital. Economic Journal 112:F45979. [aRAB]

Dyson-Hudson, N. (1966) Karimojong politics. Clarendon Press. [aRAB]

Eagly, A. H. \& Karau, S. J. (2002) Role congruity theory of prejudice toward female leaders. Psychological Review 109:573-98. [ELU]

Ebrahimi, T., Vesin, J.-M. \& Garcia, G. (2003) Brain-computer interface in multimedia communication. Signal Processing Magazine, IEEE 20(1):14-24. [EBR]

Ehrenberg, A. S. C. (1959) The pattern of consumer purchases. Journal of the Royal Statistical Society C 8:26-41. [aRAB]

Erdös, P. \& Renyí, A. (1960) On the evolution of random graphs. Publications of the Mathematical Institute of the Hungarian Academy of Sciences 5:17-61. [JES]

Eriksson, K., Enquist, M. \& Ghirlanda, S. (2007) Critical points in current theory of conformist social learning. Journal of Evolutionary Psychology 5:67-87. [aRAB]

Eriksson, K., Jansson, F. \& Sjöstrand, J. (2010) Bentley’s conjecture on popularity toplist turnover under random copying. Ramanujan Journal 23:371-96. [aRAB]

Estrada, C. A., Isen, A. M. \& Young, M. J. (1997) Positive affect facilitates integration of information and decreases anchoring in reasoning among physicians. Organizational Behavior and Human Decision Processes 72(1):117-35. [MT]

Ettredge, M., Gerdes, J. \& Karuga, G. (2005) Using web-based search data to predict macroeconomic statistics. Communications of the ACM 48:87-92. [HSM]

Evans, J. A. \& Foster, J. G. (2011) Metaknowledge. Science 331:721-25. [aRAB]

Evans, J. St. B. T. (2008) Dual-processing accounts of reasoning, judgment, and social cognition. Annual Review of Psychology 59:255-78. [H-RP]

Evans, T. \& Giometto, A. (2011) Turnover rate of popularity charts in neutral models. arXiv: $1105.4044 \mathrm{v} 1$. (Online publication) [aRAB]

Falk, E. B., Morelli, S. A., Welborn, B. L, Dambacher, K. \& Lieberman, M. D. (2013) Creating buzz: The neural correlates of effective message propagation. Psychological Science 24(7):1234-42. [MBO]

Falk, E. B., O'Donnell, M. B. \& Lieberman, M. D. (2012) Getting the word out: Neural correlates of enthusiastic message propagation. Frontiers in Human Neuroscience 6:313. [MBO]

Farmer, J. D., Patelli, P. \& Zovko, I. I. (2005) The predictive power of zero intelligence in financial markets. Proceedings of the National Academy of Sciences USA 102:2254-59. [aRAB]

Ferrer, R. A., Fisher, J. D., Buck, R. \& Amico, K. R. (2011) Pilot test of an emotional education component for sexual risk reduction. Health Psychology 30:656-60. doi: 10.1037/a0023438. [RB]

Festinger, L. (1964) Conflict, decision, and dissonance. Stanford University Press. [ANG]

Forbes, C. E. \& Grafman J. (2013) Social neuroscience: The second phase. Frontiers in Human Neuroscience 7:article 20. doi: 10.3389/fnhum.2013.00020. [EJG]

Fortunato, S. \& Castellano, C. (2007) Scaling and universality in proportional elections. Physical Review Letters 99:138701. [SF]

Frank, S. A. (2009) The common patterns of nature. Journal of Evolutionary Biology 22:1563-85. [aRAB]

Fratkin, E. M. (1989) Household variation and gender inequality in Ariaal Rendille pastoral production. American Anthropologist 91:45-55. [aRAB]

Frewer, L., van der Lans, I., Fischer, A., Reinders, M., Menozzi, D., Zhang, X., van den Berg, I. \& Zimmermann, K. (2013) Public perceptions of agri-food applications of genetic modification - A systematic review and meta-analysis. Trends in Food Science and Technology 30(2):142-52. [JEF]

Frydman, R., \& Goldberg, M. (2007) Imperfect knowledge economics. Princeton University Press. [DRo]

Gantz, J. \& Reinsel, D. (2011) Extracting value from chaos. Available at: www.emc. com/digital_universe. [aRAB]

George, H. R., Swami, V., Cornelissen, P. L. \& Tovee, M. J. (2008) Preferences for body mass index and waist-to-hip ratio do not vary with observer age. Journal of Evolutionary Psychology 6:207-18. [aRAB]

Gerow, A. \& Keane, M. T. (2011) Mining the web for the voice of the herd to track stock market bubbles. In: Proceedings of the Twenty-Second International Joint Conference on Artificial Intelligence, ed. T. Walsh, pp. 2244-49. AAAI Press. [MTK]

Gigerenzer, G. (2007) Gut feelings. The intelligence of the unconscious. Viking Penguin. [H-RP]

Gigerenzer, G. \& Gaissmaier, W. (2011) Heuristic decision making. Annual Review of Psychology 62:451-82. [XZ]

Gigerenzer, G. \& Goldstein, D. G. (1996) Reasoning the fast and frugal way: Models of bounded rationality. Psychological Review 103:650-69. [PPA]

Gigerenzer, G., Todd, P. M., \& the ABC Research Group. (1999) Simple heuristics that make us smart. Oxford University Press. [PPA]

Gigerenzer, G., Czerlinski, J. \& Martignon, L. (2002) How good are fast and frugal heuristics? In: Heuristics and biases. The psychology of intuitive judgment, T. Gilovich, D. Griffin \& D. Kahneman, pp. 559-81. Cambridge University Press. [H-RP]
Giguère, G. \& Love, B. C. (2013) Limits in decision making arise from limits in memory retrieval. Proceedings of the National Academy of Sciences USA 110:7613-18. [HSM]

Gilbert, D. T. \& Wilson, T. D. (2007) Prospection: Experiencing the future. Science 137:1351-54. [H-RP]

Gillespie, J. H. (2004) Population genetics: A concise guide, 2nd edition. Johns Hopkins University Press. [aRAB]

Gilovich, T., Griffin, D. \& Kahneman, D., eds. (2002) Heuristics and biases. The psychology of intuitive judgment. Cambridge University Press. [H-RP]

Ginsberg, J., Mohebbi, M. H., Patel, R. S., Brammer, L., Smolinski, M. S. \& Brilliant, L. (2009) Detecting influenza epidemics using search engine query data. Nature 457(7232):1012-14. [MTK, HSM]

Gintis, H. (2007) A framework for the unification of the behavioral sciences. Behavioral and Brain Sciences 30(1):1-61. [aRAB, AM]

Gintis, H. (2009) The bounds of reason: Game theory and the unification of the behavioral sciences. Princeton University Press. [aRAB]

Giving USA Foundation (2007) Giving USA 2007: Annual Report on Philanthropy for the year 2006. Giving USA Foundation. [aRAB]

Gladwell, M. (2010) Small change: Why the revolution will not be tweeted. The New Yorker, October 4, 2010, pp. 42-49. [rRAB]

Goel, S., Hofman, J. M., Lahaie, S., Pennock, D. M. \& Watts, D. J. (2010) Predicting consumer behavior with web search. Proceedings of the National Academy of Sciences USA 107:17486-90. [HSM]

Golder, S. A. \& Macy, M. W. (2011) Diurnal and seasonal mood vary with work, sleep, and daylength across diverse cultures. Science 333:1878-81. [aRAB]

Goodhardt, G. J., Ehrenberg, A. S. C. \& Chatfield, C. (1984) The Dirichlet: A comprehensive model of buying behaviour. Journal of the Royal Statistical Society A 147:621-55. [aRAB]

Google Inc. (2012) Project Glass. Available at: http://www.google.com/glass. [EBR, rRAB]

Goyal, S. (2007) Connections: An introduction to the economics of networks. Princeton University Press. [DRo]

Granovetter, M. S. (1973) The strength of weak ties. American Journal of Sociology 78:1360-80. [rRAB]

Granovetter, M. S. (1978) Threshold models for collective behavior. American Journal of Sociology 83:1420-43. [RJM]

Greve, H. R. (2003) Organizational learning from performance feedback. A behavioral perspective on innovation and change. Cambridge University Press. [SMR]

Griffiths, T. L., Kalish, M. L. \& Lewandowsky, S. (2008) Theoretical and empirical evidence for the impact of inductive biases on cultural evolution. Philosophical Transactions of the Royal Society B 363:3503-14. [AM]

Guitart-Masip, M., Barnes, G. R., Horner, A., Bauer, M., Dolan, R. J. \& Duzel, E. (2013) Synchronization of medial temporal and prefrontal rhythms in human decision making. The Journal of Neuroscience 33:442-51. doi: 10.1523/ JNEUROSCI.2573-12.2013. [EJG]

Gureckis, T. M. \& Goldstone, R. L. (2009) How you named your child: Understanding the relationship between individual decision making and collective outcomes. Topics in Cognitive Science 1:651-74. [rRAB]

Güss, C. D. \& Dörner, D. (2011) Cultural differences in dynamic decision-making strategies in a non-linear, time-delayed task. Cognitive Systems Research 12:365-76. doi: 10.1016/j.cogsys.2010.12.003. [SHN]

Hacking, I. (1992) Multiple personality disorders and its hosts. History of the Human Sciences $5(2): 3-31 . \quad[\mathrm{rRAB}, \mathrm{MC}]$

Hacking, I. (1995) The looping effect of human kinds. In: Causal cognition: A multidisciplinary debate, ed. D. Sperber, D. Premack \& A. J. Premack, pp. 35183. Oxford University Press. [MC, rRAB]

Hall, E. T. (1959) The silent language. Doubleday. [SHN]

Hamilton, M. J., Milne, B. T., Walker, R. S., Burger, O. \& Brown, J. H. (2007) The complex structure of hunter-gatherer social networks. Proceedings of the Royal Society of London B 274:2195-202. [aRAB]

Hardman, D. (2009) Judgment and decision making. Psychological perspectives. Blackwell. [H-RP]

Hauser, J. R. \& Wernerfelt, B. (1990) An evaluation cost model of consideration sets Journal of Consumer Research 16:393-408. [PPA]

Hausmann, R., Hidalgo, C. A., Bustos, S., Coscia, M., Chung, S., Jimemez, J., Simoes, A. \& Yildirim, M. A. (2011) The atlas of economic complexity: Mapping paths to prosperity. Center for International Development, Harvard University. [aRAB]

Hechtman, L., Pornpattananangkul, N. \& Y Chao, J. (2012) Psychological constructionism and cultural neuroscience. Behavioral and Brain Sciences 35(3):152-53. [ANG]

Heilman, M. E. (2001) Description and prescription: How gender stereotypes prevent women's ascent up the organizational ladder. Journal of Social Issues 57:657-74. [ELU]

Helbing, D. \& Yu, W. (2009) The outbreak of cooperation among success-driven individuals under noisy conditions. Proceedings of the National Academy of Sciences USA 106:3680-85. [aRAB]

Helbing, D., Farkas, I. \& Vicsek, T. (2000) Simulating dynamical features of escape panic. Nature 407:487-90. [aRAB] 
Hemp, P. (2009) Death by information overload. Harvard Business Review 87(9):82-89. [aRAB]

Henrich, J. (2004) Demography and cultural evolution: Why adaptive cultural processes produced maladaptive losses in Tasmania. American Antiquity 69:197214. [aRAB]

Henrich, J. (2010) The evolution of innovation-enhancing institutions. In: Innovation in cultural systems: Contributions from evolutionary anthropology, ed. M. J. O’Brien \& S. J. Shennan, pp. 99-120. MIT Press. [aRAB]

Henrich, J., and Gil-White, F. J. (2001) The evolution of prestige: Freely conferred deference as a mechanism for enhancing the benefits of cultural transmission. Evolution and Human Behavior 22:165-96. [aRAB]

Henrich, J., Boyd, R., Bowles, S., Camerer, C., Gintis, H., McElreath, R. \& Fehr, E. (2001) In search of Homo economicus: Experiments in 15 small-scale societies. American Economic Review 91:73-79. [aRAB]

Henrich, J., Boyd, R., Bowles, S., Gintis, H., Fehr, E., Camerer, C., McElreath, R., Gurven, M., Hill, K., Barr, A., Ensminger, J., Tracer, D., Marlow, F., Patton, J. Alvard, M., Gil-White F. \& Henrich, N. (2005) "Economic Man" in cross-cultural perspective: Ethnography and experiments from 15 small-scale societies. Behavioral and Brain Sciences 28:795-855. [aRAB]

Henrich, J., Heine, S. J. \& Norenzayan, A. (2010) The weirdest people in the world? Behavioral and Brain Sciences 33:61-135. [aRAB]

Henrich, J., McElreath, R., Bar, A., Ensminger, J., Barrett, C., Bolyanatz, A., Cardenas, J. C., Gurven, M., Gwako, E., Henrich, N., Lesorogol, C., Marlowe, F., Tracer, D. \& Ziker, J. (2006) Costly punishment across human societies. Science 312:1767-70. [aRAB]

Heyes, C. M. (1994) Social learning in animals: Categories and mechanisms. Biological Reviews 69:207-31. [aRAB]

Hill, K. R., Walker, R. S., Božičević, M., Eder, J., Headland, T., Hewlett, B., Hurtad, A. M., Marlowe, F., Wiessner, P. \& Wood, B. (2011) Co-residence patterns in hunter-gatherer show unique human social structure. Science 331:1286-89. [aRAB]

Hill, N. \& Alexander, J. (2006) Handbook of customer satisfaction and loyalty measurement. Gower. [aRAB]

Hill, R. A., Bentley, R. A. \& Dunbar, R. I. M. (2008) Network scaling reveals consistent fractal pattern in hierarchical mammalian societies. Biology Letters 4:748-51. [aRAB]

Hill, R. A. \& Dunbar, R. I. M. (2003) Social network size in humans. Human Nature 14:53-72. [rRAB]

Hogarth, R. M. (2010) Intuition: A challenge for psychological research on decision making. Psychological Inquiry 21:338-53. doi: 0.1080/ 1047840X.2010.520260. [H-RP]

Holland, J. \& Miller, J. (1991) Artificial adaptive agents in economic theory. American Economic Review 81(2):365-70. [RAM]

Hommes, C. (2013) Behavioral rationality and heterogeneous expectations in complex economic systems. Cambridge University Press. [rRAB]

Hoppitt, W., Kandler, A., Kendal, J. R. \& Laland, K. N. (2010) The effect of task structure on diffusion dynamics: Implications for diffusion curve and networkbased analyses. Learning \& Behavior 38:243-51. [rRAB]

Horton, J. J. (2012) Computer-mediated matchmaking: Facilitating employer search and screening. Unpublished manuscript. Available online at: http://sole-jole.org/ 13473.pdf. [ELU]

Horton, J. J., Rand, D. G. \& Zeckhauser, R. J. (2011) The online laboratory: Conducting experiments in a real labor market. Experimental Economics 14:399425. [aRAB]

Huang, J. H. \& Chen, Y. F. (2006) Herding in online product choice. Psychology and Marketing 23(5):413-28. [MTK]

Huberman, B. A. \& Adamic, L. A. (1999) Growth dynamics of the World-Wide Web. Nature 401:131. [aRAB]

Hyde, J. S. \& Linn, M. C. (2009) Gender similarities in mathematics and science. Science 314:599-600. [aRAB]

Illouz, E. (2007) Cold intimacies. The making of emotional capitalism. Polity Press. [ANG]

Ingram, H. M. \& Stern, P. C. (2007) Research and networks for decision support in the NOAA Sectoral Applications Research Program. National Academies Press. [aRAB]

Johnson, E. J. \& Goldstein, D. (2003) Do defaults save lives? Science 302:1338-39. [H-RP]

Johnson, S. D., Birks, D. J., McLaughlin, L, Bowers, K. J. \& Pease K. (2007) Prospective crime mapping in operational context: Final report. Home Office, London. [HSM]

Johnson, S. D. \& Bowers, K. J. (2004) The burglary as clue to the future: The beginnings of prospective hot-spotting. European Journal of Criminology $1: 237-55$. [HSM]

Jones, D. (2010) Human kinship, from conceptual structure to grammar. Behavioral and Brain Sciences 33:367-416. [aRAB]

Jung, D., Sul, S. \& Kiu, H. (2013) Dissociable neural processes underlying risky decisions for self versus other. Frontiers in Decision Neuroscience 7:article 15. doi: 10.3389/fnins.2013.00015. [EJG]
References/Bentley et al.: Mapping collective behavior

Kahneman, D. (2003) Maps of bounded rationality: Psychology for behavioral economics. American Economic Review 93:1449-75. [aRAB, H-RP]

Kahneman, D. (2011) Thinking, fast and slow. Penguin. [H-RP]

Kahneman, D. \& Tversky, A. (1979) Prospect theory: An analysis of decision under risk. Econometrica 47:263-91. [H-RP]

Kahneman, D. \& Tversky, A., eds. (2000) Choices, values, and frames. Cambridge University Press. [H-RP]

Kandler, A., Laland, K.N. (2009) An investigation of the relationship between innovation and cultural diversity. Theoretical Population Biology 76:59-67. [aRAB]

Kandori, M., Mailath, G. J. \& Rob, R. (1993) Learning, mutation, and long run equilibria in games. Econometrica 61:29-56. [rRAB]

Kang, S. K., Hirsh, J. B. \& Chasteen, A. L. (2010) Your mistakes are mine: Self-other overlap predicts neural response to observed errors. Journal of Experimental Social Psychology 46(1):229-32. [MBO]

Katsnelson, E., Motro, U., Feldman, M. W. \& Lotem, A. (2008) Early experience affects producer-scrounger foraging tendencies in the house sparrow. Animal Behaviour 75:1465-72. [SMR]

Kendal, R. L., Coolen, I. \& Laland, K. N. (2009) Adaptive trade-offs in the use of social and personal information. In: Cognitive Ecology II, ed. R. Dukas \& J. M. Ratcliffe, pp. 249-71. University of Chicago Press. [SMR]

Kerr, N. L. \& MacCoun, R. J. (2012) Is the leniency asymmetry really dead? Misinterpreting asymmetry effects in criminal jury deliberation. Group Processes and Intergroup Relations 15:585-602. [RJM]

Kessler D. A., Maruvka, Y. E., Ouren, J. \& Shnerb, N. M. (2012) You name it - How memory and delay govern first name dynamics. PLoS ONE 7(6):e38790. [aRAB]

Kikumbih, N., Hanson, K., Mills, A., Mponda, H. \& Schellenberg, J. A. (2005) The economics of social marketing: The case of mosquito nets in Tanzania. Social Science and Medicine 60:369-81. [aRAB]

King, G. (2011) Ensuring the data-rich future of the social sciences. Science 331:71921. [HSM]

Kitcher, P. (1993) The advancement of science. Oxford University Press. [aRAB]

Kline, M. A. \& Boyd, R. (2010) Population size predicts technological complexity in Oceania. Proceedings of the Royal Society B 277:2559-64. [aRAB]

Koehler, D. J. \& Harvey, N., eds. (2004) Blackwell handbook of judgment and decision making. Blackwell. [H-RP]

Koerper, H. C. \& Stickel, E. G. (1980) Cultural drift: A primary process of culture change. Journal of Anthropological Research 36:463-69. [aRAB]

Korzybski, A. H. S. (1933) Science and sanity: An introduction to nonAristotelian systems and general semantics. International Non-Aristotelian Library. [FLB]

Krakauer, D. (2011) Darwinian demons, evolutionary complexity, and information maximization. Chaos 21:037110. [aRAB]

Krause, J., Ruxton, G. D. \& Krause, S. (2010) Swarm intelligence in animals and humans. Trends in Ecology and Evolution 25:28-34. [SMR]

Krause, J., Wilson, A. D. M. \& Croft, D. P. (2011) New technology facilitates the study of social networks. Trends in Ecology and Evolution 26: 5-6. [SMR]

Kremers, H. M., Myasoedova E., Crowson C. S., Savova, G. Gabriel, S. E. \& Matteson, E. (2011) The Rochester Epidemiology Project: Exploiting the capabilities for population-based research in rheumatic diseases. Rheumatology (Oxford) 50(1):6-15. [CTS]

Krugman, P. (2012) The power (law) of Twitter. New York Times blogpost February 8, 2012. Available at: http://nyti.ms/zVLT72 [aRAB]

Kuiper, H., Kleter, G., Noteborn, H. \& Kok, E. (2001) Assessment of the food safety issues related to genetically modified foods. The Plant Journal 27(6):503-28. $[\mathrm{JEF}]$

Laherrère, J. \& Sornette, D. (1998) Stretched exponential distributions in nature and economy: "Fat tails" with characteristic scales. European Physical Journal B 2:525-39. [aRAB]

Laland, K. N. \& Brown, G. R. (2011) Sense and nonsense: Evolutionary perspectives on human behaviour, 2nd edition. Oxford University Press. [aRAB]

Laland, K. N. (2004) Social learning strategies. Learning and Behavior 32:4-14. [aRAB, SMR]

Laland, K. N., Odling-Smee, F. J. \& Myles, S. (2010) How culture shaped the human genome: Bringing genetics and the human sciences together. Nature Reviews Genetics 11:137-48. [aRAB]

Lampos, V. \& Cristianini, N. (2012) Nowcasting events from the social Web with statistical learning. ACM Transactions on Intelligent Systems and Technology 3 (4), Article 72:1-22. [rRAB]

Lazer, D., Pentland, A., Adamic, L., Aral, S., Barabasi, A.-L., Brewer, D., Christakis, N., Contractor, N., Fowler, J., Gutmann, M., Jebara, T., King, G., Macy, M., Roy, D. \& Van Alstyne, M. (2009) Computational social science. Science 323:721-23. [HSM]

Leckman, J. F., Feldman, R., Swain, J. E., Eicher, V., Thompson, N. \& Mayes, L. C. (2004) Primary parental preoccupation: Circuits, genes, and the crucial role of the environment. Journal of Neural Transmission 111(7):753-71. [JES] 
Lenton, A. P., Fasolo, B. \& Todd, P. M. (2008) "Shopping” for a mate: Expected vs. experienced preferences in online mate choice. IEEE Transactions on Professional Communication (Special Section: Darwinian Perspectives on Electronic Communication) 51:169-82. [aRAB]

Lenton, A. P., Fasolo, B. \& Todd, P. M. (2009) The relationship between number of potential mates and mating skew in humans. Animal Behaviour 77:55-60. [aRAB]

Lerner, J. S. \& Keltner, D. (2001) Fear, anger, and risk. Journal of Personality and Social Psychology 81:146-59. [MT]

Levine, R. (1997) A geography of time: The temporal misadventures of a social psychologist. Or how every culture keeps time just a little bit differently. Basic Books. [SHN]

Lewis, I. (1961) A pastoral democracy. Oxford University Press. [aRAB]

Lewis, K., Gonzalez, M. \& Kaufman, J. (2011) Social selection and peer influence in an online social network. Proceedings of the National Academy of Sciences USA 109:68-72. [aRAB]

Lewis, K., Kaufman, J., Gonzalez, M., Wimmer, A. \& Christakis, N. (2008) Taste, times, and ties: A new social network dataset using Facebook.com. Social Networks 30:330-42. [aRAB]

Li, J. \& Lee, L. (2009) Binary choice under social interactions: An empirical study with and without subjective data on expectations. Journal of Applied Econometrics 24:257-81. [aRAB]

Lichtenstein, S. \& Slovic, P., eds. (2006) The construction of preference. Cambridge University Press. [H-RP]

Lieberman, E., Hauert, C. \& Nowak, M. A. (2005) Evolutionary dynamics on graphs. Nature 433:312-16. [aRAB]

Lieberman, E., Michel, J.-B., Jackson, J., Tang, T. \& Nowak, M. A. (2007) Quantifying the evolutionary dynamics of language. Nature 449:713-16. [aRAB]

Lieberman, M. D. (2010) Social cognitive neuroscience. In: Handbook of social psychology, 5th edition, ed. S. T. Fiske, D. T. Gilbert \& G. Lindzey, pp. 143-93. McGraw-Hill. [MBO

Lindeyer, C. M., Meaney, M. J. \& Reader, S. M. (2013) Early maternal care predicts reliance on social learning about food in adult rats. Developmental Psychobiology 55:168-75. [SMR]

Lindquist, J. D. \& Kaufman-Scarborough, C. J. (2007) The Polychronic-Monochronic Tendency Model: PMTS scale development and validation. Time and Society 16(2/3):269-301. doi: 10.1177/0961463X07080270. [SHN]

Loewenstein, G. (2000) Emotions in economic theory and economic behavior American Economic Review 90:426-32. [MT]

Loewenstein, G., ed. (2007) Exotic preferences: Behavioral economics and human motivation. Oxford University Press. [H-RP]

Loewenstein, G. \& Lerner, J. S. (2003) The role of affect in decision making. In: Handbook of affective science, ed. R. J. Davidson, H. H. Goldsmith \& K. R. Scherer, pp. 619-42. Oxford University Press. [H-RP]

Loewenstein, G. F., Weber, E. U., Hsee, C. K. \& Welch, N. (2001) Risk as feelings. Psychological Bulletin 127(2):267-86. doi: 10.1037/0033-2909.127.2.267. [RB]

Loftus, E. F. \& Pickrell, J. E. (1995) The formation of false memories. Psychiatric Annals 25:720-25. [H-RP]

Lorenz J., Rauhut, H., Schweitzer, F. \& Helbing, D. (2011) How social influence can undermine the wisdom of crowd effect. Proceedings of the National Academy of Sciences USA 108:9020-25. [aRAB]

Louis, W. R., Taylor, D. M. \& Douglas, R. L. (2005) Normative influence and rational conflict decisions: Group norms and cost-benefit analyses for intergroup behavior. Group Processes and Intergroup Relations 8(4):355-74. [XZ]

Low, B. S. (2001) Why sex matters: A Darwinian look at human behavior. Princeton University Press. [aRAB]

MacCoun, R. J. (2012) The burden of social proof: Shared thresholds and social influence. Psychological Review 119:345-72. [RJM]

MacCoun, R. J., Cook, P., Muschkin, C. \& Vigdor, J. (2008) Distinguishing spurious and real norm effects: Evidence from artificial societies, small-group experiments, and real schoolyards. Review of Law and Economics 4(3):695-14 $[\mathrm{RJM}]$

Mace, R. \& Colleran, H. (2009) Kin influence on the decision to start using modern contraception: A longitudinal study from rural Gambia. American Journal of Human Biology 21:472-77. [aRAB]

Malmgren, R. D., Ottino, J. M. \& Nunes Amaro, L. A. (2010) The role of mentorship in protégé performance. Nature 465:622-26. [aRAB]

Manski, C. F. (1993) Identification of endogenous social effects: The reflection problem. Review of Economic Studies 60:531-42. [arRAB]

Marchetti, C. \& Drent, P. J. (2000) Individual differences in the use of social information in foraging by captive great tits. Animal Behaviour 60:131-40. [SMR]

Marshall, E. (2000) Reinventing an ancient cure for malaria. Science 290:437-39. [aRAB]

Masucci, A. P., Kalampokis, A., Martínez Equíluz, V. \& Hernández-García, E. (2011) Wikipedia information flow analysis reveals the scale-free architecture of the semantic space. PLoS ONE 6(2):e17333. [aRAB]
Mateos P., Longley, P. A. \& O'Sullivan, D. (2011) Ethnicity and population structure in personal naming networks. PLoS ONE 6(9):e22943. [aRAB]

Mayes, L. C., Swain, J. E. \& Leckman, J. F. (2005) Parental attachment systems: Neural circuits, genes, and experiential contributions to parental engagement. Clinical Neuroscience Research 4(5/6):301-13. doi: 10.1016/j.cnr.2005.03.009. [JES]

McCain, R. A., Hamilton, R. \& Linnehan, F. (2011) The problem of emergency department overcrowding: Agent-based simulation and test by questionnaire. In: Lecture Notes in Economics and Mathematical Systems 652: Emergent results of artificial economics, ed. S. Osinga, G. J. Hofstede, \& T. Verwaart, pp. 91-102. Springer Verlag. [RAM]

McCarty, N. M., Poole, K. T. \& Rosenthal, H. (2006) Polarized America: The dance of ideology and unequal riches. MIT Press. [aRAB]

McClain, D. L. (2010) No. 1 player takes on the world, and wins. The New York Times, September 12, 2010, p. A32. [SMR]

McFadden, D. L. (2001) Economic choices. American Economic Review 91:35178. [aRAB]

McKelvey, R. D. \& Palfrey T. R. (1995) Quantal response equilibria for normal form games. Games and Economic Behavior 10:6-38. [rRAB]

McLuhan, M. (1964) Understanding media: The extensions of man. McGraw-Hill. $[\mathrm{RB}, \mathrm{rRAB}]$

McNamara, J. M. \& Fawcett, T. W. (2012) Optimal strategies and heuristics for ecological search problems. In: Cognitive search: Evolution, algorithms, and the brain, ed. P. M. Todd, T. T. Hills \& T. W. Robbins, pp. 301-15. MIT Press. [SMR]

Mesoudi, A. (2008) An experimental simulation of the "copy-successful-individuals" cultural learning strategy: Adaptive landscapes, producer-scrounger dynamics, and informational access costs. Evolution and Human Behavior 29:350-63. [arRAB, AM]

Mesoudi, A. (2009) The cultural dynamics of copycat suicide. PLoS ONE 4:e7252. $[\mathrm{AM}]$

Mesoudi, A. (2010) The experimental study of cultural innovation. In: Innovation in cultural systems: Contributions from evolutionary anthropology, ed. M. J. O’Brien \& S. J. Shennan, pp. 175-91. MIT Press. [rRAB]

Mesoudi, A. (2011) Cultural evolution: How Darwinian theory can explain human culture and synthesize the social sciences. University of Chicago Press. [aRAB, AM]

Mesoudi, A. \& Lycett, S. J. (2009) Random copying, frequency-dependent copying and culture change. Evolution and Human Behavior 30:41-48. [aRAB, AM]

Mesoudi, A. \& O'Brien, M. J. (2008a) The cultural transmission of Great Basin projectile-point technology I: An experimental simulation. American Antiquity 73:3-28. [AM, rRAB]

Mesoudi, A. \& O'Brien, M. J. (2008b) The cultural transmission of Great Basin projectile point technology II: An agent-based computer simulation. American Antiquity 73:627-44. [AM, rRAB]

Mesoudi, A., Whiten, A. \& Laland, K. N. (2006) Towards a unified science of cultural evolution. Behavioral and Brain Sciences 29:329-83. [aRAB, AM]

Michel, J. B., Shen, Y. K., Aiden, A. P., Veres, A., Gray, M. K., Googles Book Team, Pickett, J. P., Hoiberg, D., Clancy, D., Norvig, P., Orwant, J., Pinker, S., Nowak, M. A. \& Aiden, E. L. (2011) Quantitative analysis of culture using millions of digitized books. Science 331(6014):176-82. [MTK]

Mielby, H., Sandøe, P. \& Lassen, J. (2012) The role of scientific knowledge in shaping public attitudes to GM technologies. Public Understanding of Science 22(155):155-68. [JEF]

Milot, E., Mayer, F. M., Nussey, D. H., Boisvert, M., Pelletier, F. \& Réale D. (2011) Evidence for evolution in response to natural selection in a contemporary human population. Proceedings of the National Academy of Sciences USA 108:17040-45. [aRAB]

Mitchell, T. M. (2009) Mining our reality. Science 326:1644-45. [HSM]

MMG (Miniwatts Marketing Group) (2013) Asia Internet use, population data and Facebook statistics. [Online publication.] Available at: http://www.internetworldstats.com/stats.htm $\quad[\mathrm{SHN}]$

Moat, H. S., Curme, C., Avakian, A., Kenett, D. Y., Stanley, H. E. \& Preis, T. (2013 Quantifying Wikipedia usage patterns before stock market moves. Scientific Reports 3:1801. [HSM]

Mohler, G. O., Short, M. B., Brantingham, P. J., Schoenberg, F. P. \& Tita, G. E. (2011) Self-exciting point process modeling of crime. Journal of the American Statistical Association 106:100-08. [HSM]

Monmonier, M. (1996) How to lie with maps, 2nd edition. University of Chicago Press. [RJM]

Montrey, M. \& Shultz, T. R. (2010) Evolution of social learning strategies. In: Proceedings of the Ninth IEEE International Conference on Development and Learning, Ann Arbor, MI, August 18-21, 2010, pp. 95-100. IEEE. [DRu]

Morris, S. \& Shin, H.-S. (2003) Global games: Theory and applications. In: Advances in economics and econometrics, vol. 1: Theory and applications, Eighth World Congress, ed. M. Dewatripont, L. Hansen \& S. Turnovsky, pp. 56-114. Cambridge University Press. [DRo]

Moussaïd, M., Kämmer, J. E., Analytis, P. \& Neth, H. (2013) Social influence and the collective dynamics of opinion formation. PLoS ONE 8(11). [PPA] 
Nagle, T. T. \& Holden, R. K. (2002) The strategy and tactics of pricing. PrenticeHall. [aRAB]

National Health Services (2013) NHS “Choices” webpage. Available at: www.nhs.uk/ conditions/Chronic-fatigue-syndrome/Pages/Introduction.aspx (consulted April 14, 2013). [CTS]

Nettle, D. (2009) Ecological influences on human behavioural diversity: A review of recent findings. Trends in Ecology and Evolution 24:618-24. [aRAB]

Nettle, D. (2010) Understanding of evolution may be improved by thinking about people. Evolutionary Psychology 8:205-28. [aRAB]

Nestle, M. \& Nesheim, M. (2012) Why calories count: From science to politics. University of California Press. [aRAB]

Newman, M. E. J. (2005) Power laws, Pareto distributions and Zipf's law. Contemporary Physics 46:323-51. [aRAB]

Norgate, S. H., Winstanley, C., Davies, N., Smith, L., Harding, M., Speed, C., et al. (2013) Smarter cities for pedestrians: Innovating real-time information systems to promote walking school buses. Paper presented at DE2013: Open DigitalThe Fourth Annual Digital Economy All Hands Meeting. MediaCity, UK, Salford, November 4-6, 2013. [SHN]

O'Brien, M. J. \& Bentley, R. A. (2011) Stimulated variation and cascades: Two processes in the evolution of complex technological systems. Journal of Archaeological Method and Theory 18:309-35. [arRAB]

O'Brien, M. J., Darwent, J. \& Lyman, R. L. (2001) Cladistics is useful for reconstructing archaeological phylogenies: Palaeoindian points from the southeastern United States. Journal of Archaeological Science 28:1115-36. [AM]

O'Connor, B., Balasubramanyan, R., Routledge, B. \& Smith, N. (2010) From tweets to polls: Linking text sentiment to public opinion time series. In: Proceedings of the Fourth International AAAI Conference on Weblogs and Social Media [ICWSM 2010], Washington DC, May 23-26, 2010, ed. M. Hearst, W. Cohen \& S. Gosling, pp. 122-29. AAAI Press. [MBO]

Ohtsuki, H., Hauert, C., Lieberman, E. \& Nowak, M. A. (2006) A simple rule for the evolution of cooperation on graphs and social networks. Nature 441:502-505. [aRAB]

Olivola, C. Y. \& Sagara, N. (2009) Distributions of observed death tolls govern sensitivity to human fatalities. Proceedings of the National Academy of Sciences USA 106:22151-156. [HSM]

Onnela, J.-P. \& Reed-Tsochas, F. (2010) Spontaneous emergence of social influence in online systems. Proceedings of the National Academy of Sciences USA 107:18375-80. [aRAB, SF, MTK]

Ordónez, L. D., Connolly, T. \& Coughlan, R. (2000) Multiple reference points in satisfaction and fairness assessment. Journal of Behavioral Decision Making 13:329-44. [H-RP]

Ormerod, P. (2012) Positive linking: How networks can revolutionise the world. Faber and Faber. [aRAB]

Ouattara, K., Lemasson, A. \& Zuberbühler, K (2009) Campbell's monkeys use affixation to alter call meaning. PLoS ONE 4(11):e7808. [aRAB]

Palmer, C. R. \& Kristan, W. B., Jr. (2011) Contextual modulation of behavioral choice. Current Opinion in Neurobiology 21:520-26. doi: 101016/j. conb.2011.05.003. [EJG]

Payne, J. W. (1982) Contingent decision behavior. Psychological Bulletin 92:382402. [ELU]

Payne, J. W., Bettman, J. R. \& Johnson, E. J. (1988) Adaptive strategy selection in decision making. Journal of Experimental Psychology: Learning, Memory, and Cognition 14:534-52. [ELU]

Payne, J. W., Bettman, J. R. \& Johnson, E. J. (1993) The adaptive decision maker Cambridge University Press. [ELU]

Pennebaker, J. W. (2011) The secret life of pronouns: What our words say about us. Bloomsbury Press. [MBO]

Perry, E. L., Davis-Blake, A. \& Kulik, C. T. (1994) Explaining gender-based selection decisions: A synthesis of contextual and cognitive approaches. Academy of Management Review 19(4):786-820. [ELU]

Pfister, H.-R. \& Böhm, G. (2008) The multiplicity of emotions: A framework of emotional functions in decision making. Judgment and Decision Making 3(1):517. $[\mathrm{H}-\mathrm{RP}]$

Pfister, H.-R. \& Böhm, G. (2012) Responder feelings in a three-player three-option ultimatum game: Affective determinants of rejection behavior. Games 3(1):129. $[\mathrm{H}-\mathrm{RP}]$

Pillai, J. S., Schmidt, C. T. A. \& Richir, S. (2013) Achieving presence through evoked reality. Frontiers in Psychology 4:86. doi: 10.3389/fpsyg.2013.00086. [CTS]

Pleger, B. \& Villringer, A. (2013) The human somatosensory system: From perception to decision making. Progress in Neurobiology 103:76-97. [EJG]

Plous, S. (1993) The psychology of judgment and decision making. McGraw-Hill. [aRAB]

Postmes, T., Spears, R. \& Cihangir, S. (2001) Quality of decision making and group norms. Journal of Personality and Social Psychology 80:918-30. [aRAB]

Powell, A., Shennan, S. \& Thomas, M. G. (2009) Late Pleistocene demography and the appearance of modern human behavior. Science 324:1298-301. [aRAB]
Preis, T., Moat, H. S. \& Stanley, H. E. (2013) Quantifying trading behavior in financial markets using Google Trends. Nature Scientific Reports 3, No. 1684, pp. 1-6. [HSM, rRAB]

Preis, T., Moat, H. S., Stanley, H. E. \& Bishop, S. R. (2012) Quantifying the advantage of looking forward. Nature Scientific Reports 2, No. 350. [HSM, rRAB]

Preis, T., Reith, D. \& Stanley, H. E. (2010) Complex dynamics of our economic life on different scales: Insights from search engine query data. Philosophical Transactions of the Royal Society A 368:5707-19. [HSM]

Rand, D. G. (2012) The promise of Mechanical Turk: How online labor markets can help theorists run behavioral experiments. Journal of Theoretical Biology 299:172-79. [aRAB]

Rand, D. G., Dreber, A., Ellingsen, T., Fudenberg, D. \& Nowak, M. A. (2009) Positive interactions promote public cooperation. Science 325:1272-75. [aRAB]

Ratkiewicz, J., Fortunato S., Flammini, A., Menczer F. \& Vespignani, A. (2010) Characterizing and modeling the dynamics of online popularity. Physical Review Letters 105:158701. [SF]

Reader, S. M. (2004) Distinguishing social and asocial learning using diffusion dynamics. Learning and Behavior 32(1):90-104. [SMR, DRu]

Reali, F. \& Griffiths, T. L. (2010) Words as alleles: Connecting language evolution with Bayesian learners to models of genetic drift. Proceedings of the Royal Society B 277:429-36. [aRAB]

Rendell, L., Fogarty, L., Hoppitt, W. J. E., Morgan, T. J. H., Webster, M. M. \& Laland, K. N. (2011) Cognitive culture: Theoretical and empirical insights into social learning strategies. Trends in Cognitive Sciences 15:68-76. [aRAB, $\mathrm{SMR}$ ]

Repetto, R. (2006) Punctuated equilibrium and the dynamics of U.S. environmental policy. Yale University Press. [aRAB]

Richerson, P. J. \& Boyd, R. (2005) Not by genes alone. University of Chicago Press. [AM]

Rogers, E. M. (1962) Diffusion of innovations. Free Press. [aRAB]

Romer, P. (2012) Process, responsibility, and Myron's Law. In: In the wake of the crisis: Leading economists reassess economic policy, ed. O. J. Blanchard, D. Romer, A. M. Spence \& J. E. Stigltiz, pp. 111-24. MIT Press. [aRAB]

Saavedra, S., Reed-Tsochas, F. \& Uzzi, B. (2009) A simple model of bipartite cooperation for ecological and organizational networks. Nature 457:463-66. [aRAB]

Salganik, M. J., Dodds, P. S. \& Watts, D. J. (2006) Experimental study of inequality and unpredictability in an artificial cultural market. Science 311(5762):854-56. doi:10.1126/science.1121066. [arRAB, SF, GLM, MTK, SMR]

Salzman, P. C. (1999) Is inequality universal? Current Anthropology 40:31-61 [aRAB]

Samuelson, P. (1947) Foundations of economic analysis. Enlarged edition (1983). Harvard University Press. [DRo]

Savage, L (1954) The foundations of statistics. Wiley. [DRo]

Saxe, R. \& Kanwisher, N. (2003) People thinking about thinking people: fMRI studies of Theory of Mind. NeuroImage 19(4):1835-42. [MBO]

Scheffer, M., Carpenter, S. R., Lenton, T. M., Bascompte, J., Brock, W., Dakos, V., van der Koppel, J., van de Leemput, I. A., Levin, S. A., van Nes, E. H., Pascual, M. \& Vandermeer, J. (2012) Anticipating critical transitions. Science 338:34448. $[\mathrm{rRAB}]$

Schelling, T. (1960) The strategy of conflict. Harvard University Press. [RAM]

Schelling, T. (1969) Models of segregation. The American Economic Review 59:48893. [RJM]

Schmidt, C. T. A. \& Kraemer, F. (2006) Robots, Dennett and the autonomous: A terminological investigation. Minds and Machines 16(1):73-80. [CTS]

Sela, A. \& Berger, J. (2012) Decision quicksand: How trivial choices suck us in. Journal of Consumer Research 39:360-70. [aRAB]

Shalizi, C. R. \& Thomas, A. C. (2010) Homophily and contagion are genetically confounded in observational social network studies. Sociological Methods and Research 40:211-39. [arRAB]

Shennan, S. J. (2000) Population, culture history and the dynamics of culture change. Current Anthropology 41:811-35. [aRAB]

Shiller, R. J. (1995) Conversation, information and herd behaviour. American Economic Review 85:181-85. [MTK]

Shultz, T. R. (2003) Computational developmental psychology. MIT Press. [DRu, $\mathrm{rRAB}]$

Silberzahn, R., Uhlmann, E. L. \& Zhu, L. (under review) Pay as she goes: For stereotypically male jobs, women tend to be hired by the hour. [ELU]

Simkin, M. V. \& Roychowdhury, V. P. (2003) Read before you cite! Complex Systems 14:269. [aRAB]

Simon, H. A. (1955) A behavioral model of rational choice. Quarterly Journal of Economics 69:99-118. [aRAB, HSM]

Simon, H. A. (1956) Rational choice and the structure of the environment. Psychological Review 63:129-38. [PPA]

Smith, V. (2008) Rationality in economics. Cambridge University Press. [DRo]

Snyder, J. P. (1993) Flattening the earth: Two thousand years of map projections. University of Chicago Press. [RJM] 
Sparrow, B., Liu, J. \& Wegner, D. M. (2011) Google effects on memory: Cognitive consequences of having information at our fingertips. Science 333:776-78. [aRAB]

Sporns, O. (2012) From simple graphs to the connectome: Networks in neuroimaging. Neuroimage 62(2):881-86. Available at: http://www.ncbi.nlm.nih.gov/ pubmed/21964480 [JES]

Sporns, O., Tononi, G. \& Edelman, G. M. (2002) Theoretical neuroanatomy and the connectivity of the cerebral cortex. Behavioral and Brain Science 135 (1-2):69-74. Available at: http://www.ncbi.nlm.nih.gov/pubmed/12356436 [JES]

Sripada, C., Angstadt, M., Kessler, D., Phan, K. L., Liberzon, I., Evans, G. W., Welsh, R., Kim P. \& Swain, J. E. (2013) Volitional regulation of emotions produces distributed alterations in connectivity between visual, attention control, and default networks. Neuroimage 89:110-21. doi: 10.1016/j.neuroimage.2013.11.006. [Epub ahead of print] [JES]

Stahl, F., Gaber, M. M., Bramer, M. \& Yu, P. S. (2010) Pocket Data Mining: Towards collaborative Data Mining in mobile computing environments. In: Proceedings of the Twenty-Second IEEE International Conference on Tools with Artificial Intelligence (ICTAI), Arras, France, 27-29 October, 2010, ed. É. Grégoire, pp. 323-30. IEEE Computer Society. [EBR]

Stasser, G. \& Davis, J. H. (1981) Group decision making and social influence: A social interaction sequence model. Psychological Review 88(6):523-51. [XZ]

Steenhaut, S. \& Van Kenhove, P. (2006) The mediating role of anticipated guilt in consumers' ethical decision-making. Journal of Business Ethics 69:269_88. [MT]

Stephens, D. W. (1991) Change, regularity, and value in the evolution of animal learning. Behavioral Ecology 2:77-89. [SMR]

Stewart, N. (2009) Decision by sampling: The role of the decision environment in risky choice. Quarterly Journal of Experimental Psychology 62:1041-62. [HSM]

Stewart, N., Chater, N. \& Brown, G. D. A. (2006) Decision by sampling. Cognitive Psychology 53:1-26. [HSM]

Strauss, S. (2012) Six reasons why political polarization will only get worse. Huffington Post (October 14). Available at: http://www.huffingtonpost.com/stevenstrauss/megatrend-six-reasons-ame_b_1965182.html. [aRAB]

Stringer, M. J., Sales-Pardo, M. \& Amaral, L. A. N. (2010) Statistical validation of a global model for the distribution of the ultimate number of citations accrued by papers published in a scientific journal. Journal of the American Society for Information Science and Technology 61:1377-85. [aRAB]

Sumecki, D., Chipulu, M. \& Ojiako, U. (2011) Email overload: Exploring the moderating role of the perception of email as a "business critical" tool. International Journal of Information Management 31:407-14. [aRAB]

Sumpter, D. J. T., Mann, R. P. \& Perna, A. (2012) The modelling cycle for collective animal behaviour. Interface Focus 2:764-73. [SMR]

Supekar, K. \& Menon, V. (2012) Developmental maturation of dynamic causal control signals in higher-order cognition: A neurocognitive network model. PLoS Computational Biology 8(2): e1002374. Available at: http://dx.plos.org/ 10.1371/journal.pcbi.1002374.t001. [JES]

Surowiecki, J. (2004) The wisdom of crowds: Why the many are smarter than the few. Abacus. [aRAB]

Surowiecki, J. (2005) The wisdom of crowds. Anchor Books. [MTK]

Swain, J. E. (2011) The human parental brain: In vivo neuroimaging. Progress in Neuro-Psychopharmacology and Biological Psychiatry 35(5):1242-54. Available at: http://www.ncbi.nlm.nih.gov/entrez/query.fcgi?cmd=Retrieve\&db=PubMed\&dopt $=$ Citation\&list_uids $=21036196 \quad$ [JES]

Swain, J. E., Kim, P. \& Ho, S. S. (2011) Neuroendocrinology of parental response to baby-cry. Journal of Neuroendocrinology 23(11):1036-1041. Available at: http:// www.ncbi.nlm.nih.gov/entrez/query.fcgi? $\mathrm{cmd}=$ Retrieve\&db=PubMed\&dopt=Citation\&list_uids $=21848646$ [JES]

Swain, J. E., Konrath, S., Brown, S. L., Finegood, E. D., Akce, L. B., Dayton, C. J. \& Ho, S. S. (2012) Parenting and beyond: Common neurocircuits underlying parental and altruistic caregiving. Parenting Science and Practice 12(2-3):115-23. Available at: http://www.ncbi.nlm.nih.gov/pubmed/22971776 [JES]

Swain, J. E. \& Lorberbaum, J. P. (2008) Imaging the human parental brain. In: Neurobiology of the parental brain, ed. R. Bridges, pp. 83-100. Academic Press. [JES]

Swain, J. E., Mayes, L. C. \& Leckman, J. F. (2004) The development of parent-infant attachment through dynamic and interactive signaling loops of care and cry. Behavioral and Brain Sciences 27(4):472-73. [JES]

Tausczik, Y. \& Pennebaker, J. W. (2010) The psychological meaning of words: LIWC and computerized text analysis methods. Journal of Language and Social Psychology 29:24-54. [rRAB]

Thaler, R. H. \& Sunstein, C. R. (2008) Nudge: Improving decisions about health, wealth, and happiness. Penguin Group. [H-RP]

Timbeau, X. (2012) The fireman and the architect. In: What's right with macroeconomics?, ed. R. Solow \& J.-P. Touffut, pp. 8-32. Edward Elgar. [DRo]

Timmermans, D. (1993) The impact of task complexity on information use in multiattribute decision making. Journal of Behavioral Decision Making 6:95-111. [ELU]
Tizzoni, M., Bajardi, P., Poletto, C., Ramasco, J. J., Balcan, D., Gonçalves, B., Perra, N., Colizza, V. \& Vespignani, A. (2012) Real-time numerical forecast of global epidemic spreading: Case study of 2009 A/H1N1pdm. BMC Medicine 10:165. [HSM]

Toelch, U., Bruce, M. J., Meeus, M. T. H. \& Reader, S. M. (2011) Social performance cues induce behavioral flexibility in humans. Frontiers in Psychology 2:160. [SMR]

Toelch, U., van Delft, M. J., Bruce, M. J., Donders, R., Meeus, M. T. H. \& Reader, S. M. (2009) Decreased environmental variability induces a bias for social information use in humans. Evolution and Human Behavior 30:32-40. [SMR]

Toffler, A. (1970) Future shock. Random House. [aRAB]

Toivonen, R., Castelló, X., Eguíluz, V. M., Saramäki, J., Kaski, K. \& San Miguel, M. (2009) Broad lifetime distributions for ordering dynamics in complex networks. Physical Review E 79:016109. [SF]

Tomasello, M., Carpenter, M., Call, J., Behne, T. \& Moll, H. (2005) Understanding and sharing intentions: The origins of cultural cognition. Behavioral and Brain Sciences 28:675-735. [aRAB]

Tomasello, M., Kruger, A. C. \& Ratner, H. H. (1993) Cultural learning. Behavioral and Brain Sciences 16:495-511. [aRAB]

Tomlin, D., Nedic, A., Prentice, D. A., Holmes, P. \& Cohen, J. D. (2013) The neural substrates of social influence on decision making. PloS One 8(1): e52630. [XZ]

Tsetsos, K., Chater, K. \& Usher, M. (2012) Salience driven value integration explains decision biases and preference reversal. Proceedings of the National Academy of Sciences USA 109:9659-64. [aRAB]

Tsuji, Y. (2006) Railway Time and Rubber Time: The paradox in the Japanese conception of time. Time and Society 15(2/3):177-95. doi: 10.1177/ 0961463X06066950. [SHN]

Tucker, D. M. (1981) Lateral brain function, emotion, and conceptualization. Psychological Bulletin 89(1):19-46. doi: 10.1037/0033-2909.89.1.19. [RB]

Tversky, A. \& Simonson, I. (1993) Context-dependent preferences. Management Science 39:1179-89. [H-RP]

Tversky, A. \& Thaler, R. H. (1990) Anomalies: Preference reversals. Journal of Economic Perspectives 4:201-11. [H-RP]

Twenge, J., Campbell, K. W. \& Gentile, B. (2012) Increases in individualistic words and phrases in American books, 1960-2008. PLoS ONE 7(7):e40181. [aRAB]

United Nations (2006) World urbanization prospects: The 2005 revision. United Nations. [aRAB]

Venditti, C., Meade, A. \& Pagel, M. (2010) Phylogenies reveal new interpretation of speciation and the Red Queen. Nature 463:349-52. [aRAB]

Vespignani, A. (2009) Predicting the behavior of techno-social systems. Science 325:425-28. [HSM]

Viswanath, B., Mislove, A., Cha, M. \& Gummadi, K. P. (2009) On the evolution of user interaction in Facebook. In: Proceedings of the 2nd ACM Workshop on Online Social Networks, ed. J. Crowcroft \& B. Krishnamurthy, pp. 37-42. Association for Computing Machinery. [aRAB]

Voeks, R. A. (1996) Tropical forest healers and habitat preference. Economic Botany 50:381-400. [aRAB]

Vyssotski, A. L., Dell'Omo, G., Dell'Ariccia, G., Abramchuk, A. N., Serkov, A. N., Latanov, A. V., Loizzo, A., Wolfer, D. P. \& Lipp, H. P. (2009) EEG responses to visual landmarks in flying pigeons. Current Biology 19:1159-66. [SMR]

Watts, D. J. (2011) Everything is obvious: Once you know the answer. Atlantic Books. [SF]

Watts, D. J. \& Hasker, S. (2006) Marketing in an unpredictable world. Harvard Business Review 84(9):25-30. [arRAB]

Watts, D. J. \& Strogatz, S. H. (1998) Collective dynamics of "small-world' networks. Nature 393(6684):440-42. Available at: http://www.ncbi.nlm.nih.gov/pubmed/ 9623998 [JES, rRAB]

Wegner, D. M. (1995) A computer network model of human transactive memory. Social Cognition 13:319-39. [aRAB]

Williams, S. J. (2000) Is rational choice theory "unreasonable"? The neglected emotions. In: Rational choice theory: Resisting colonization, ed. M. S. Archer \& J. Q. Tritter, pp. 57-72. Routledge. [ANG]

Winterhalder, B., Kennett, D. J., Grote, M. N. \& Bartruff, J. (2010) Ideal free settlement of California's Northern Channel Islands. Journal of Anthropological Archaeology 29:469-90. [aRAB]

Winterhalder, B. \& Smith, E. A. (2000) Analyzing adaptive strategies: Human behavioral ecology at twenty-five. Evolutionary Anthropology 9:51-72. [aRAB]

Wright, P. (1975) Consumer choice strategies: Simplifying vs. optimizing. Journal of Marketing Research 12:60-67. [ELU]

Wright, S. (1932) The roles of mutation, inbreeding, crossbreeding and selection in evolution. Proceedings of the Sixth International Congress of Genetics 1:35666. $[\mathrm{AM}]$

Wu, F. \& Huberman, B. A. (2007) Novelty and collective attention. Proceedings of the National Academy of Sciences USA 104:17599-601. [aRAB] 
References/Bentley et al.: Mapping collective behavior

Yu, A. J. \& Dayan, P. (2005) Uncertainty, neuromodulation, and attention. Neuron 46: 681-92. doi: 00/101016/j.neuron.2005.04.026. [EJG]

Yu, S., Huang, D., Singer, W. \& Nikolić, D. (2008) A small world of neuronal synchrony. Cerebral Cortex 18(12):2891-901. Available at: http://www.ncbi.nlm. nih.gov/pubmed/18400792 [JES]

Yule, G. U. (1924) A mathematical theory of evolution based on the conclusions of Dr. J. C. Willis. Philosophical Transactions of the Royal Society B 213:21-87. [aRAB]

Zeelenberg, M. \& Pieters, R. (2006) Feeling is for doing: A pragmatic approach to the study of emotions in economic behavior. In: Social psychology and economics, ed. D. DeCremer, M. Zeelenberg \& J. K. Murnighan, pp. 117-37. Erlbaum. [H-RP
Zerubavel, E. (1982) The standardization of time: A sociohistorical perspective. The American Journal of Sociology 88(1):1-23. [SHN]

Zhou, X.-Y. (2013) The reciprocal effect of social influence and decision making. Unpublished raw data. [XZ]

Zimbardo, P. G. \& Boyd, J. N. (1999) Putting time in perspective: A valid, reliable individual differences metric. Journal of Personality a Social Psychology 77(6):1271-88. [SHN]

Zipf, G. K. (1949) Human behavior and the principle of least effort. AddisonWesley. [aRAB]

Zun, L. S., Blume, D. N., Lester, J., Simpson, G. \& Downey, L. (2004) Accuracy of emergency medical information on the web. American Journal of Emergency Medicine 22:94-97. [aRAB] 